2015-07-09

\title{
A 3D Multiscale Model of Chemotaxis in Bacteria
}

\author{
$\mathrm{Wu}$, Andrew
}

Wu, A. (2015). A 3D Multiscale Model of Chemotaxis in Bacteria (Unpublished master's thesis).

University of Calgary, Calgary, AB. doi:10.11575/PRISM/24626

http://hdl.handle.net/11023/2345

Downloaded from PRISM Repository, University of Calgary 


\title{
UNIVERSITY OF CALGARY
}

A 3D Multiscale Model of Chemotaxis in Bacteria

by

Andrew $\mathrm{Wu}$

\begin{abstract}
A THESIS
SUBMITTED TO THE FACULTY OF GRADUATE STUDIES

IN PARTIAL FULFILLMENT OF THE REQUIREMENTS FOR THE

DEGREE OF MASTER OF SCIENCE
\end{abstract}

GRADUATE PROGRAM IN COMPUTER SCIENCE

CALGARY, ALBERTA

June, 2015

(c) Andrew Wu 2015 


\begin{abstract}
We present a multiscale 3D model of a colony of E. coli bacteria. We simulate four distinct yet computationally interconnected levels.

In the first and second level, we simulate chemical diffusion in the environment to capture the colony populations chemotactic behaviour. The bacterium interact with a discrete grid which models diffusion of chemicals. The first level presents this population behaviour in the form of a colour gradient, and proceeding to the second level we present the behaviour as particles.

The third level, the chemotactic motions of the individual bacterium is presented. And in the fourth level, the cellular processes that drive the chemotactic behaviour is presented.

We show four interconnected model layers that capture the biological processes from the colony layer down to the level of interacting molecules. The aim of this work is to construct a platform that enhances understanding of natural life by serving as a valuable educational tool.
\end{abstract}




\section{Acknowledgements}

I would like to thank my mentor and supervisor, Dr. Christian Jacob, and my fellow peers and colleagues in the LINDSAY Virtual Human lab at the University of Calgary for their patience, mentorship, and insights.

Everyone's encouragement, enthusiasm, and support has pushed me beyond my limits and gave me the confidence I needed during this time.

My family for their unconditional support and their belief in my abilities.

Finally, my beloved girlfriend for her companionship, and patience with me through these years. 


\section{Table of Contents}

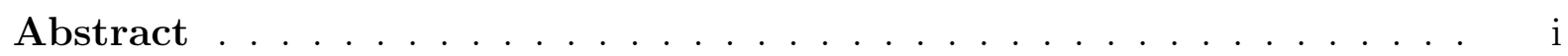

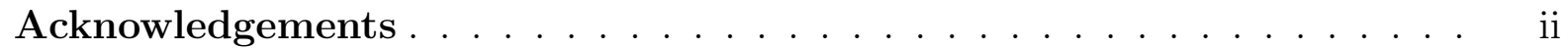

Table of Contents . . . . . . . . . . . . . . . . . . . . . . . iii

List of Tables . . . . . . . . . . . . . . . . . . . . . . . v v

List of Figures . . . . . . . . . . . . . . . . . . . . . . . . . . . vi vi

List of Symbols . . . . . . . . . . . . . . . . . . . . . . . . $\mathrm{x}$

1 Introduction . . . . . . . . . . . . . . . . . . . . . . . . . 1

1.1 The Existing Project $\ldots \ldots \ldots \ldots \ldots \ldots$

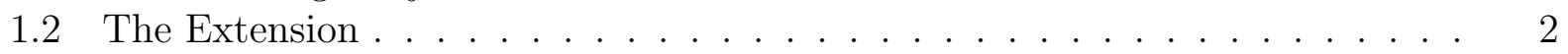

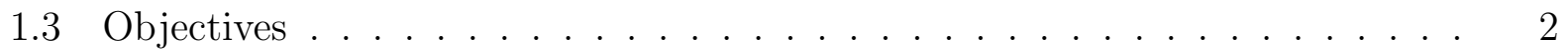

1.3.1 Milestones . . . . . . . . . . . . . . . . . . . . 5

1.4 Additional Components Added to The Original Model . . . . . . . . . . . 6

2 BIOLOGY: Escherichia coli and Chemotaxis . . . . . . . . . . . . . . 9 9

$2.1 \quad$ Escherichia coli $\ldots \ldots \ldots \ldots \ldots$

2.2 Chemotaxis $\ldots \ldots \ldots \ldots \ldots \ldots$

2.2 .1 Chemotaxis and E. coli $\ldots \ldots \ldots \ldots$

2.3 Chemotaxis in E. coli . . . . . . . . . . . . . . . . . . . . . . . 13

$2.3 .1 \quad$ Excitation Response - Signal Transduction Cascade . . . . . . . . . . 18

2.3 .2 Adaptation Response - Methylation . . . . . . . . . . . . . . . . . . . 19

$2.3 .3 \quad$ Negative Feedback Loop - Adaptation . . . . . . . . . . . . . . . . . 19

3 Chemotaxis Models . . . . . . . . . . . . . . . . . . . . . . . . . . . . 23

3.1 Previous Models of Chemotaxis . . . . . . . . . . . . . . . . . . 23

3.2 Mathematical Models . . . . . . . . . . . . . . . . . . . . . . . . . . . . . 27

3.3 Agent-based Models $\ldots \ldots \ldots$. . . . . . . . . . . . . . . . . . . . . . . . . . . 29

3.4 Hybrid Models $\ldots \ldots \ldots \ldots$. . . . . . . . . . . . . . . . . . . . . . . . . . . 32

$3.5 \quad$ Multiscale Modelling . . . . . . . . . . . . . . . . . . . . . . . . . . 33

4 The Escherichia coli Chemotaxis Model . . . . . . . . . . . . . . . . . . . . 34

4.1 Architecture of the Model . . . . . . . . . . . . . . . . . . . . . 34

4.1 .1 Hybrid Modeling in Our Model . . . . . . . . . . . . . . . . . 36

4.2 Layers of the Model and Functionality _. . . . . . . . . . . . . . . . . 37

4.2 .1 Layer One and Layer Two $\ldots \ldots \ldots$

4.2 .2 Third Layer $\ldots \ldots \ldots \ldots$

4.2 .3 Fourth Layer $\ldots \ldots \ldots$

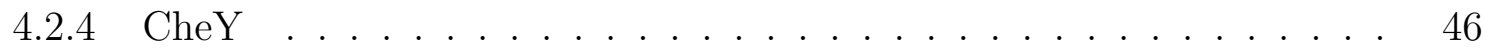

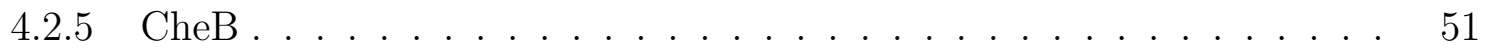

$4.2 .6 \quad$ CheZ and CheR . . . . . . . . . . . . . . . . . . . . . . . . . . . . . 54

4.2 .7 CheAW Complex and Receptor . . . . . . . . . . . . . . . . . 54

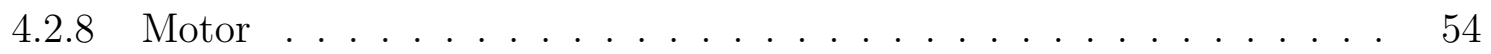

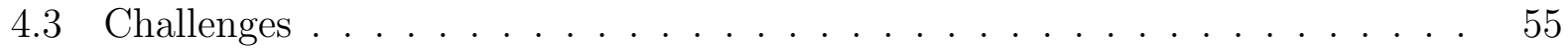

$4.3 .1 \quad$ Biological Challenges $\ldots \ldots \ldots \ldots \ldots$

4.3 .2 Technical Challenges $\ldots \ldots \ldots \ldots \ldots$

4.3 .3 Combined Challenges . . . . . . . . . . . . . . . . . . . . . . . . . 58 


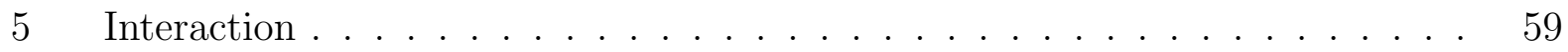

$5.1 \quad$ The Proteins $\ldots \ldots \ldots \ldots$

5.2 The World $\ldots \ldots \ldots \ldots$

5.3 Adding and Removing Agents . . . . . . . . . . . . . . . . . . 61

5.4 The Speed $\ldots \ldots \ldots \ldots$. . . . . . . . . . . . . . . . . . . . . . . . . . . . . . . 63

5.5 Travelling Through the Layers . . . . . . . . . . . . . . . . . . . . . 64

$6 \quad$ Results . . . . . . . . . . . . . . . . . . . . . . . . . . . . 68

$6.1 \quad$ Multiscale and Interaction Interface $\ldots \ldots \ldots \ldots$

6.1 .1 Protein Interaction $\ldots \ldots \ldots \ldots$

6.1 .2 Multiscale Interaction . . . . . . . . . . . . . . . . . . . 74

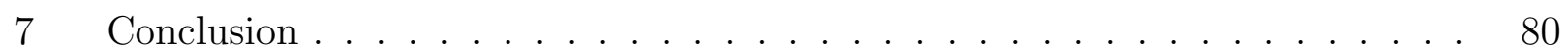

$8 \quad$ Future Work . . . . . . . . . . . . . . . . . . . . . . . . . . . . . . . . . . 82

Bibliography . . . . . . . . . . . . . . . . . . . . . . . . . . . . . 84

A $\quad$ Lindsay Platform . . . . . . . . . . . . . . . . . . . . . . . . . . . . . . . . 91

A.1 Lindsay Composer . . . . . . . . . . . . . . . . . . . . . . . . . . . . . . . . . 91

B $\quad$ Rendering \& Visualization $\ldots \ldots \ldots \ldots$

B.1 Protein Visualization . . . . . . . . . . . . . . . . . . . . . . . . . . . . . . . 95

B.1.1 Importing PDB Files . . . . . . . . . . . . . . . . . . . . . 96

C Importing 3D Models . . . . . . . . . . . . . . . . . . . . . 100

C.1 Importing PDB Structures Into Lindsay Composer . . . . . . . . . . . . 100

C.2 Model Appearance . . . . . . . . . . . . . . . . . . . . . . . . . . . 101 


\section{List of Tables}

$2.1 \quad$ Chemotaxis Proteins and Their Roles in the Pathway. . . . . . . . . . . . . . 18

$3.1 \quad$ A Chronological History of Chemotaxis Models and Their Emphasis . . . . . 27 


\section{List of Figures and Illustrations}

0.1 Snapshots of the four layers of resolution within our multiscale $E$. coli model. Each view progressively reveals more details of chemotaxis. . . . . . . . . . . xiii

0.2 Screen captures from the interactive "Cell Size and Scale" module of the Genetic Science Learning Centre [31], presenting a basic multiscale model displaying a magnification of an area, and as the magnification proceeds more minuscule objects are revealed. Size of Coffee bean to the size of an E. coli

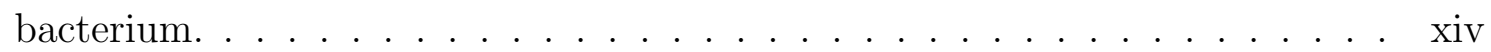

0.3 Agent-based modelling in action. Screen capture from Esmaeili's Journey Inside the Cell simulation [27]. Printed with permission from Afshin Esmaeili.] xv

0.4 The 4 divisional layers of the multiscale model . . . . . . . . . . . . xvi

1.1 A) Bacterium cell in a saturated or non-stimulus environment (Random Walk). B) Bacterium cell in an environment where the repellent stimulus is stronger on the right side (Represented by gradual increase in red colour alpha value from left to right), C) Bacterium cell in environment where attractant stimulus exists and comes from the right side (Represented by the gradual decrease in green colour alpha value from right to left). . . . . . . . . . . . . . . 3

1.2 Architecture of the connections between each of the layers, the interface and the data behind each of the layers. . . . . . . . . . . . . . . . . . 8

2.1 Screen captures of chemotactic movement toward an attractant stimulus shown at time step $1(\mathrm{~T}=1)$, and time step $2(\mathrm{~T}=2)$ during the simulation. $\mathrm{A})$ Gradient visualization under the naked-eye view (layer one), B) Zoomed in colony view (layer two) of the same scene. $\ldots \ldots \ldots . . \ldots 11$

2.2 Screen captures of chemotactic movement away from a repellent stimulus shown at time step $1(\mathrm{~T}=1)$, and time step $2(\mathrm{~T}=2)$ during the simulation. A) Gradient visualization under the naked-eye view (layer one), B) Zoomed in colony view (layer two) of the same scene. . . . . . . . . . . . 12

2.3 A) A tryptone soft agar plate in which motile cells can swim through waterfilled tunnels in the agar. Two chemotactic colonies are shown. As the cells grow, they establish attractant gradients through consumption energy sources saturated in the agar ${ }^{1}$. B) Representation of chemotactic ring in layer one of our multiscale model (naked-eye view). C) Representation of chemotactic ring in layer two of our multiscale model (colony view). . . . . . . . . . . . . . . 14

2.4 Flagella filaments of the E. coli bacterium. . . . . . . . . . . . . . . . . . . 15

2.5 A) The flagella filaments rotate CCW, they bundle together and propel the bacterium forward. B) The flagella filaments rotate $\mathrm{CW}$, breaking the bundle and causing the bacterium to tumble. . . . . . . . . . . . 16

2.6 A) The Motor location from outside the E. coli bacterium. B) The Motor location from inside the E. coli bacterium. . . . . . . . . . . . . . . . . . . . 16

2.7 Chemotactic Pathways: schematics and translations into our agent-based $E$. coli model. A.1) Excitation. A.2) Agent interactions for excitation. . . . . . 21 
2.8 Chemotactic Pathways: schematics and translations into our agent-based $E$. coli model. A.1) Adaptation. A.2) Agent dynamics during adaptation response. 22

4.1 Communication hierarchy between the components in the model. The interface and views run in the world, which is the simulation environment. The data model is situated behind the scenes. Transition only occurs with the immediate views beside them, such that the naked-eye view cannot transition to the molecular view, and vice versa. All data between the views is communicated via the interface, there is no direct communication between the actual

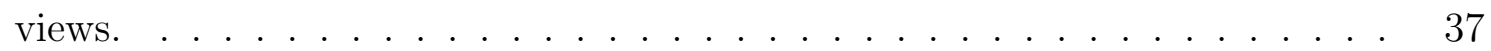

4.2 Brief overview on how the hybrid model system operates within our multiscale model. The first two layers, Naked-Eye View and Colony View, rely on mathematical modeling. The other two layers, Individual View and Molecular View, rely on agent-based modeling, along with an overlap between mathematical and agent-based in the Colony view and Individual view. . . . . . . . . . . 38

4.3 A) What the simulation environment looks like at the start of the simulation $(\mathrm{T}=0)$. B) and $\mathrm{C})$ What the simulation looks like at a time step after the simulation has been executed $(\mathrm{T}=1) \mathrm{B}$ ) shows the first layer (naked-eye) $\mathrm{C}$ ) shows the second layer (colony). . . . . . . . . . . . . . . . . . 42

4.4 A) E. coli gradient in the naked-eye view. B) Attractant gradient in the naked-eye view. C) Repellent gradient in the naked-eye view. . . . . . . . . . 43

4.5 A) E. coli chemotactic movement towards an attractant stimulus in the nakedeye view over several time steps (T). B) E. coli chemotactic movement away from repellent stimulus in environment in the naked-eye view over several time steps (T). . . . . . . . . . . . . . . . . . . . . . . . . 44

4.6 The agent-Based representation of the excitation response. A) The CheY agent is active and moving towards the CheAW Complex. B) Upon collision with the CheAW Complex, CheY becomes phosphorylated and changes conformation to CheY-P. C) and D), the CheY-P agent moves away from the complex and towards the motor at the end of the bacterium. Finally, E) and F), CheY-P collides with the motor, upon collision CheY-P is bounded to the motor until it is dephosphorylated by CheZ. . . . . . . . . . . . . . . . . 47

4.7 The agent-Based representation of the Adaption response. CheR, CheR-M, CheB, CheB-P surrounds the vicinity of the CheAW Complex and Receptor. When CheR-M collides with the receptor, the M attaches to the receptor and CheR-M changes conformation to CheR. It will then wander away and after a period of time it becomes CheR-M again and moves towards the receptor to attempt to methylate it again. CheB moves towards the CheAW Complex to become phosphorylated. Once phosphorylated, CheB changes conformation to CheB-P and wanders for a period of time, and then it approaches the receptor to demethylate it. Behind the scenes, the simulation tracks the methyl groups currently attached to the receptor, and if the receptor has too many groups it will refuse the CheR-M from methylating it further. . . . . . . . . . . . . . 48

4.8 A glimpse of what it should look like inside a real bacterium. Several thousands of proteins. . . . . . . . . . . . . . . . . . 49 
4.9 A table of our chemotaxis Proteins as agents in the molecular layer of the simulation. The agents meshes were imported using the associated PDB ID from the PDB (http://www.rcsb.org/.)] . . . . . . . . . . . . 50

5.1 The Simulation Toolbar in Lindsay Composer used to control elements (Attractant, Repellent, E. coli ) in the naked-eye and colony view of the Model, and the Time Control bar to control the speed of simulation. . . . . . . . . . 62

5.2 Process of adding new (pre-defined) agents from the Lindsay Composer's Skyroom into the Simulation Environment. Here, we are adding a CheB agent into the environment by selecting the Skyroom from the Composer's Toolbar and then dragging in our agent into the environment. . . . . . . . . . . . . . 63

5.3 Molecular View - Inside the cell in the cytoplasm. . . . . . . . . . . . . . . . 65

5.4 The molecular view with the particle system off and with the particle system

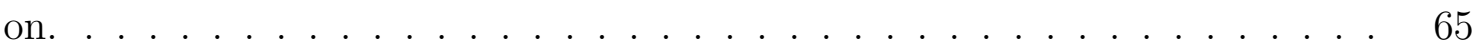

5.5 A) Layer one (naked-eye view) of our multiscale model, shown is an single $E$. coli colony with an uniform/no stimulus environment. B) Layer two (colony view) of our multiscale model, rather than the E. coli gradient, we see the actual bacterium cells in the environment. . . . . . . . . . . . . . . . . . 66

5.6 Molecular details of the chemotaxis pathway with the particle systems turned off to reduce the density of elements as compared to Figure 4 4.8, . . . . . . . 67

6.1 A capillary assay invented by W.Pfeffer in the 1880's. The test chemical diffuses from the capillary mouth, establishing a steep gradient that attracts bacteria to the entrance. The cells enter the capillary and are subsequently documented by colony counts. ${ }^{2} \ldots \ldots \ldots$. . . . . . . . . . . 69

6.2 A) Figure redrawn from Figure 2b, from Team Goettingen, in the 2012 iGEM competition (http://2012.igem.org/Team:Goettingen/Notebook/Results). The figure shows the result of 4 strains of $E$. coli growing in the presence of an attractant, where one can see the chemotactic ring formation and the general growth direction of the $E$. coli in the presence of an attractant stimulus. B) Movement of the $E$. coli colony (blue) towards an attractant over two time steps $(\mathrm{T}=1, \mathrm{~T}=2)$ in our model. $\ldots \ldots \ldots \ldots . \ldots . \ldots 70$

6.3 Continuous addition of attractant stimulus in different locations. With each new addition, the E. coli moves towards the new high concentration of the

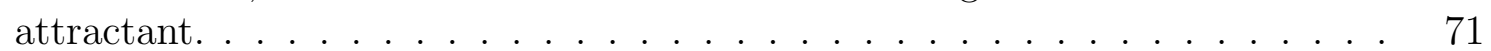

$6.4 \quad$ E. coli as a single agent in agar environment. . . . . . . . . . . . . . . . . . 72

6.5 Embedded Interface directly into the LINDSAY Composer Simulation Envi-

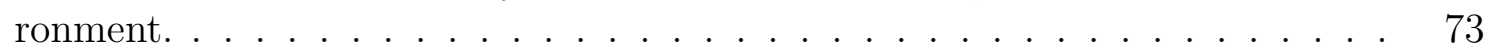

6.6 Snapshots of the four interfaces for each of our views within the multiscale $E$.

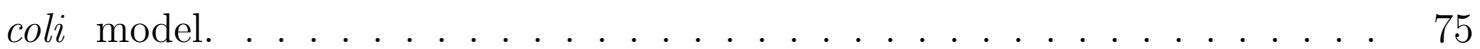

6.7 A) CheAW in the default state (On). B) CheAW in disabled (Malfunction) state (Off). . . . . . . . . . . . . . . . . . . . . 75

6.8 A) With CheAW active, we can see normal phosphorylation events do occur for CheB and CheY resulting in CheB-P and CheY-P. B) With CheAW inactive, no phosphorylation occurs. . . . . . . . . . . . . . . 76 
6.9 With CheZ disabled, no phosphorylation can occur, leading to all proteins such as CheY to be phosphorylated. . . . . . . . . . . . . . . . 76

6.10 Disabled receptor agent maintained through Eye (view 1) to Molecular (view 4) transitions in the multiscale model. . . . . . . . . . . . . . . . . . . 77

6.11 A) Disabled receptor, we can see how the $E$. coli colony ignores the repellent stimulus beside it as it does not distinctly run away as compared with B) where the receptor is functional and we can see that the E. coli colony moves away from the repellent. . . . . . . . . . . . . . . . . 78

6.12 Bacterium running straight into the repellent. With the receptor disabled, there is no signal to phosphorylate the CheY. This leads to a higher concentration of CheY which suppresses tumbling. . . . . . . . . . . . . . 79

A.1 Component hierarchy and structure of a cube (A) and the cube's visual appearance in a scene (B) viewed through a camera. The scene includes graphics, physics and transform information of a cube in simulation space. . . . . . . . 92

A.2 Components can communicate with each other. For example, physics component can update the graphics indirectly through a transform component. The update is bidirectional and the graphics component could update the transform which in turn will result in the physics to be updated. The Scene in the Figure illustrates the Physics, Transform, and Graphics interaction while the simulation is running. . . . . . . . . . . . . . . . . . 93

A.3 Screenshot of Lindsay Composer's simulation environment. The interface is composed of a toolbar, scene and simulation area. . . . . . . . . . . . . . . . 94

B.1 Three-Dimensional structure of Chemotaxis proteins and their corresponding PDB ID number. . . . . . . . . . . . . . . . . . . . . . . 96

B.2 Steps required to convert a PDB file to OGRE mesh. . . . . . . . . . . . . 97

B.3 Screenshot of mMaya import tab toolkit (A). mMaya's protein tab (B) allows for different visualization (mesh vs atoms) options to render a protein structure on the screen. . . . . . . . . . . . . . . . . . . . . . . . 98

B.4 Screenshot of a CheY Protein rendered on screen in Autodesk Maya after importing a PDB file through mMaya toolkit. . . . . . . . . . . . . . . . . . 99

C.1 A screenshot of OGRE media directories loaded by the Composer's resource

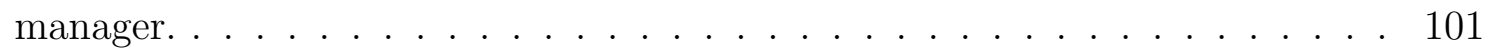

C.2 A example of the mesh and material files associated and required to render a Protein (CheY) in our LINDSAY Composer environment. A) The rendered result of the CheY protein in the simulation. B) The mesh file that defines the mesh data and points to the material file that defines rendering options. C) A snippet of the material script for the CheY protein. . . . . . . . . . . . 103 


\section{List of Symbols, Abbreviations and Nomenclature}

\begin{tabular}{|c|c|}
\hline Symbol & Definition \\
\hline $\mathrm{U}$ of $\mathrm{C}$ & University of Calgary \\
\hline $\mathrm{ABM}$ & Agent Based Modelling \\
\hline $\mathrm{ABS}$ & Agent Based System \\
\hline IBM & Individual Based Modelling \\
\hline CheY & Chemotaxis Protein Y \\
\hline CheY-P & Chemotaxis Protein Y with Phosphate Group \\
\hline CheZ & Chemotaxis Protein Z \\
\hline CheZ-P & Chemotaxis Protein Z with Phosphate Group \\
\hline CheB & Chemotaxis Protein B \\
\hline CheB-P & Chemotaxis Protein B with Phosphate Group \\
\hline CheR & Chemotaxis Protein R \\
\hline CheR-M & Chemotaxis Protein R with Methyl Group \\
\hline CheAW & Chemotaxis Protein $\mathrm{A}+\mathrm{W}$ \\
\hline $\mathrm{PDB}$ & Protein Data Bank \\
\hline $\mathrm{LC}$ & LINDSAY Composer \\
\hline OGRE & Object-oriented Graphics Rendering Engine \\
\hline $\mathrm{CCW}$ & Counter Clockwise \\
\hline $\mathrm{CW}$ & Clockwise \\
\hline ODE & Ordinary differential equations \\
\hline PDE & Partial differential equations \\
\hline $3 \mathrm{D}$ & Three-Dimensional \\
\hline $2 \mathrm{D}$ & Two-Dimensional \\
\hline
\end{tabular}




\section{Preface}

Growing up, I have witnessed the growth and evolution of technology. I found it extremely fascinating learning how technology was utilized in society. It was the use of technology to model different systems that captured my interest in this field. As technology evolved, so did its uses. I often saw simulations in many diverse fields except for Biology. It was not until I entered University that I first encountered the infusion of technology into Biology.

During these past years, learning about Biology and cellular processes has been interesting. However, these cellular processes are often described using mathematical models (Chapter 3.2). This made it hard for me to fully comprehend the concepts and I found myself struggling to understand the system as it involved complex differential equations [57]. To someone that has a weak background in mathematics - such as myself - this became an issue. In the process of searching for a simpler way of understanding the models, I discovered a technique known as agent-based modelling (explained in Chapter 3.3). This technique simulates actions and interactions of the autonomous agents to assess their effects on the system as a whole.

The idea of using agent-based modelling in biology intrigued me. Apart from agent-based modelling, I also wanted to incorporate the idea of multiscale into the model itself. Imagine a constant magnification of some targeted object, and with each level of magnification more details are revealed. Figure 0.1 demonstrates how I envisioned this concept. The Genetic Science Learning Centre [31] is an example of this multiscale idea. On their website [31], the animation named "Cell Size and Scale" delivers a great overview of multiscale as shown in Figure 0.2 .

Each individual learns differently, and the individual learning styles can generally be categorized into one of three styles: auditory, visual, and kinaesthetic [39]. For myself, I noticed that I learn best when I physically interact with the topic I am learning. Later, I learned 
that this meant I am a kinaesthetic learner. Although I excelled in biology, there was always an obstacle for me in comprehending the cellular processes as they are usually described with mathematical models. Of course, textbooks served as a great learning resource, and when I was in grade school, textbooks were essentially all we had. Imagine if we could bring the textbook to life, such that we could read, hear, visualize, and interact with the contents, this would essentially improve the ability to learn any topic by anyone as it covers all three learning styles. Now imagine learning about a cellular process by executing a program that simulates it, presents it visually, and provides us with the ability to interact in real-time, rather than simply reading a description of the process.

This idea led me to a former student of Christian Jacob, Afshin Esmaeili. Esmaeili's thesis served as the foundation of my thesis. His thesis involved the modelling of molecular details of the lac-operon pathway inside a prokaryotic cell [27]. Esmaeili utilized agent-based modelling with mathematical modelling to simulate a biological model. Figure 0.3 shows the use of agent-based modelling in action. Esmaeili's thesis was based on a highly visual artistic book by David Goodsell titled "The Machinery of Life" [32]. Esmaeili coined his thesis "A Journey Inside the Cell" as his thesis turned Goodsell's highly detailed coloured visual images of the cellular processes of the lac-operon into an interactive simulation.

I decided to extend Esmaeili's model in two ways. First, I incorporated another biological pathway into the model utilizing agent-based modelling and secondly I created a multiscale model. As shown in Figure 0.4, this multiscale model consists of four levels. Each one of these levels represents a different layer in the model that is distinguished by its magnification. These levels correspond to different details seen at different magnification levels. Imagine looking at a colony of cells with the naked eye (naked-eye view), then under a microscope with 10x zoom (colony view), then 20x (individual view), and 100x (molecular view) as shown in Figure 0.1 . 


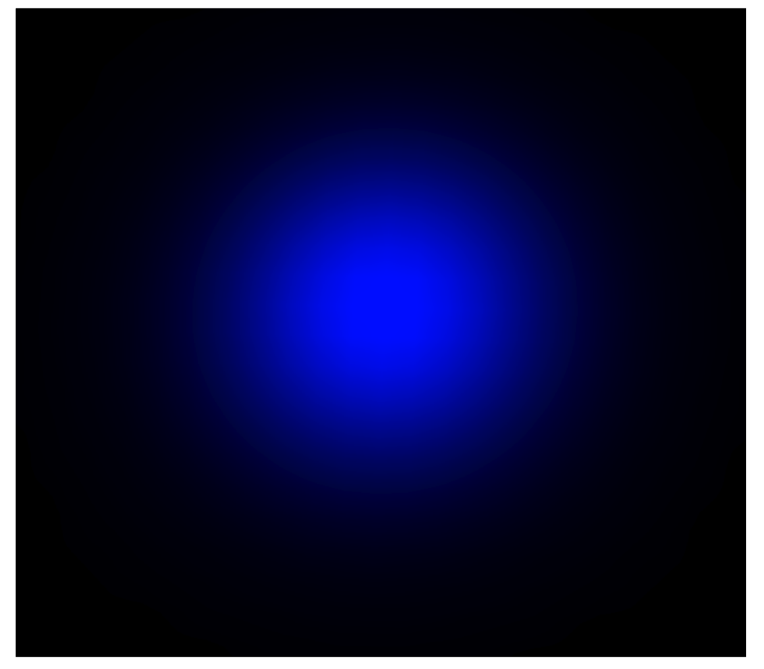

Naked-eye View: agar plate

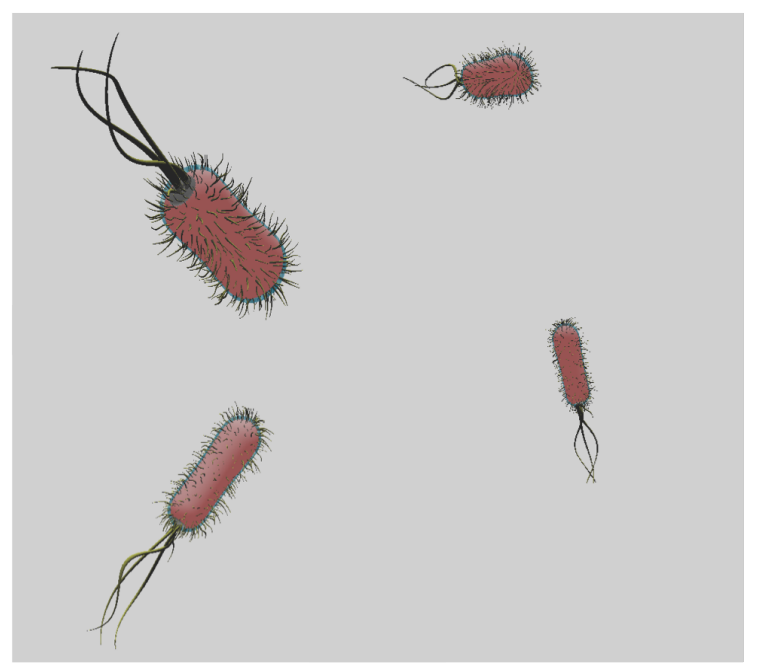

Individual View: E. coli

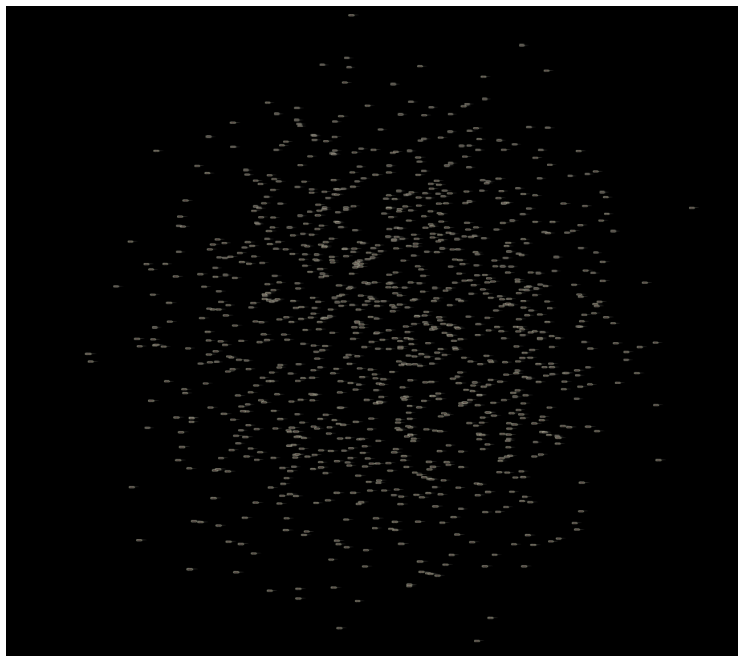

Colony View: cell cluster

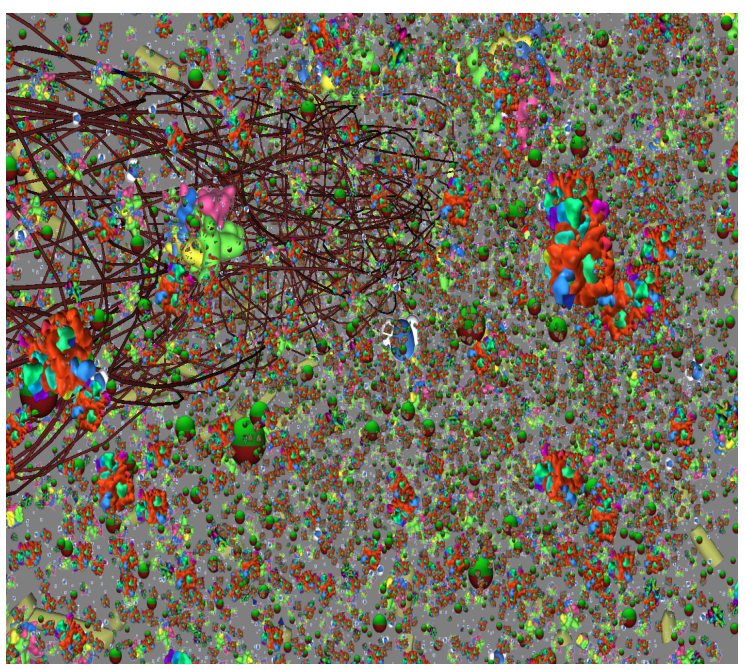

Molecular View: cytoplasm

Figure 0.1: Snapshots of the four layers of resolution within our multiscale E. coli model. Each view progressively reveals more details of chemotaxis. 


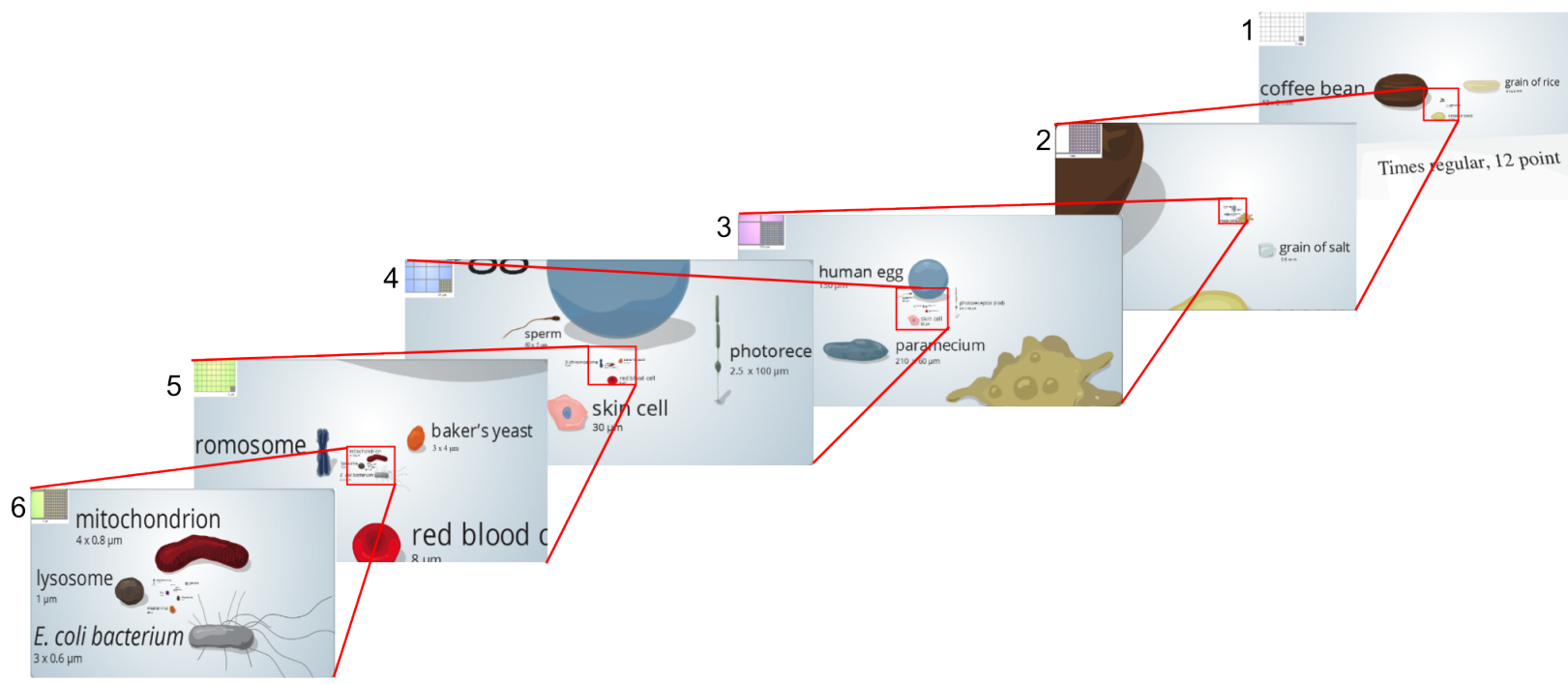

Figure 0.2: Screen captures from the interactive "Cell Size and Scale" module of the Genetic Science Learning Centre [31], presenting a basic multiscale model displaying a magnification of an area, and as the magnification proceeds more minuscule objects are revealed. Size of Coffee bean to the size of an E. coli bacterium. 


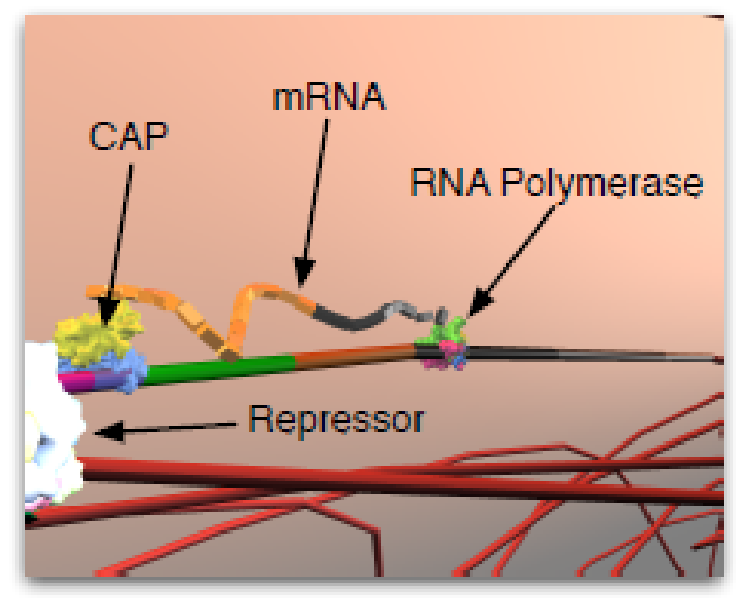

A

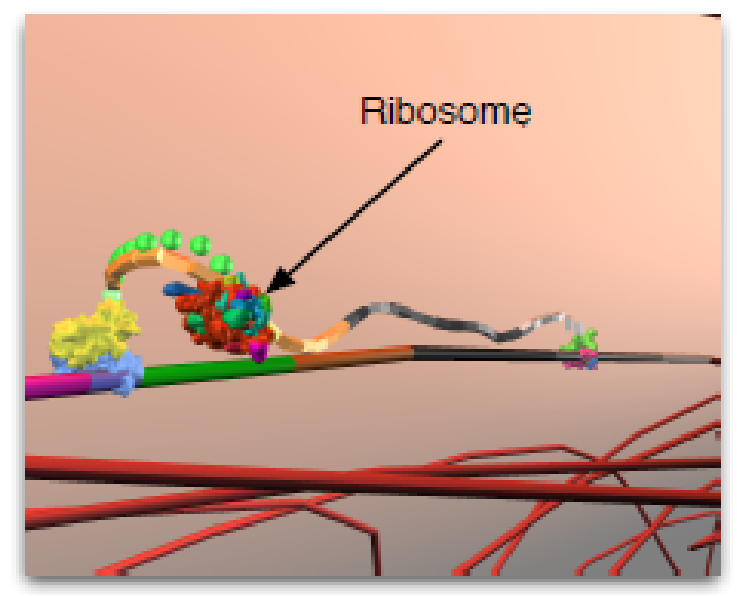

B

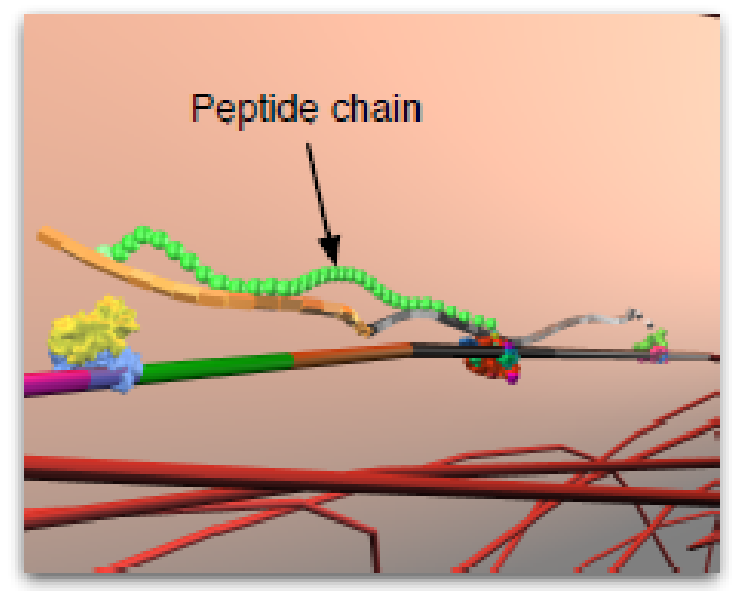

$\mathrm{C}$

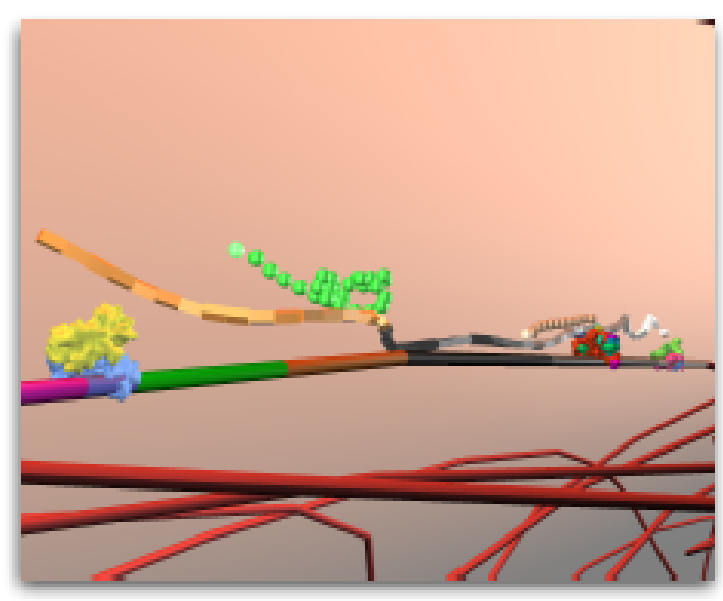

D

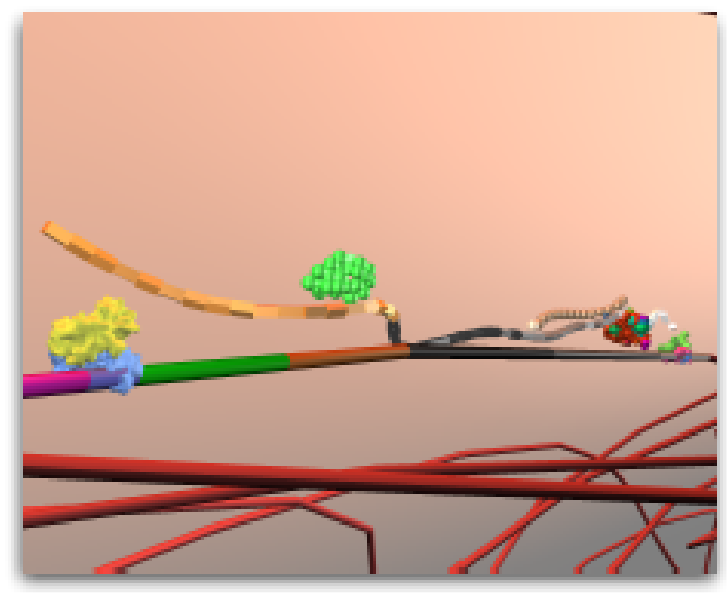

E

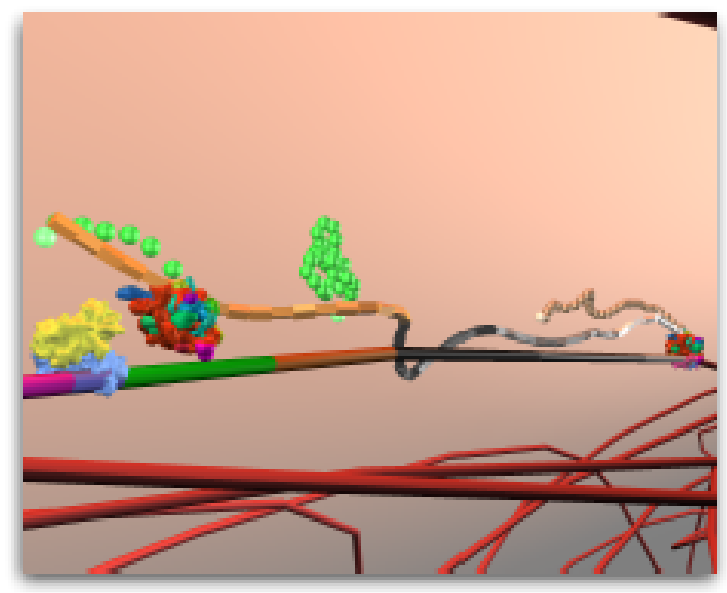

F

Figure 0.3: Agent-based modelling in action. Screen capture from Esmaeili's Journey Inside the Cell simulation [27]. Printed with permission from Afshin Esmaeili. 


\section{Layer 1}

\section{Naked-Eye View}

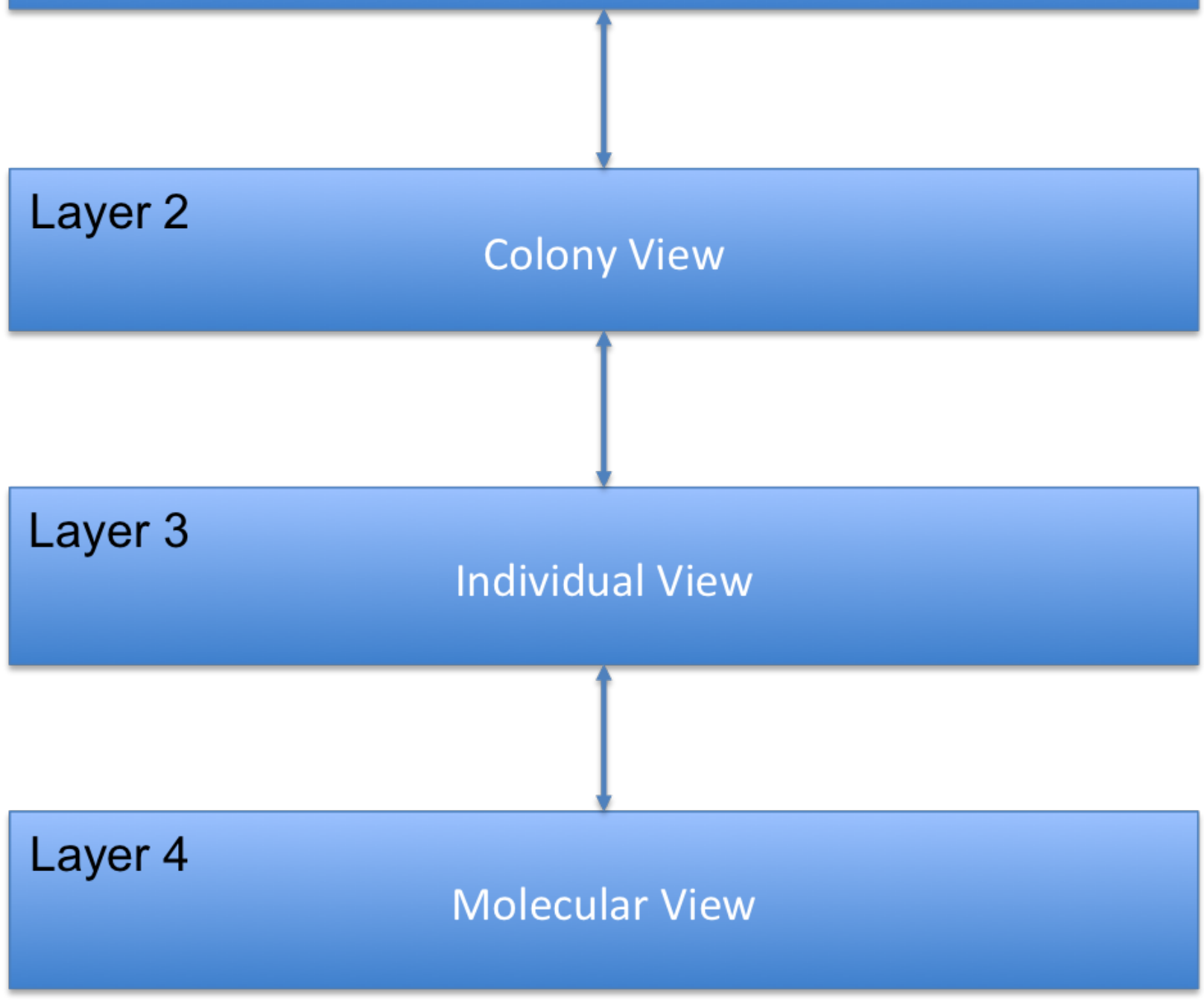

Figure 0.4: The 4 divisional layers of the multiscale model 


\section{Chapter 1}

\section{Introduction}

\subsection{The Existing Project}

Afshin Esmaeili's thesis focused on visualizing the cellular processes inside a prokaryotic cell [27]. His research focused on the lac-operon pathway. Unlike most simulations where the cellular processes are played out similar to a movie - such as the Bio-Visions Project [15] Esmaeili's model allowed for interactions with the cellular processes during the simulation.

The Bio-Visions project [15] targets education through the use of animations to visualize the beauty of biological processes. Utilizing powerful animation and graphic rendering, the Bio-Visions project created appealing animations illustrating how biological processes function. However, it lacked interactivity with the user.

"A Journey Inside the Cell" took the missing component and incorporated it into the simulation. The simulation was created through a combination of agent-based and mathematical modelling. This hybrid approach of switching between two modelling methods dramatically reduces computation resource cost to execute the model [27]. The two modelling methods will be further discussed in Chapter 3 .

In Esmaeili's model, the molecular level of the cellular process of the lac-operon pathway was extensively modelled. The detail in the molecular level is startlingly impressive; however, this was just the beginning. There are many more pathways that can be incorporated into the cell, and with the evolution of technology new methods can be used to simulate the model. 


\subsection{The Extension}

"A Journey Inside the Cell" focused on the lac-operon pathway. This journey is a beautiful master piece of work that brings the cellular process of the lac-operon to life. The activity of transcription, translation, and protein folding is visually projected in a way that captivates the user and enhances the overall experience of learning about the lac-operon. This model is fully interactive which differentiates it from other models of the cellular processes.

My extension of this work was to incorporate the journey inside with the journey outside of the cell. My primary focus for this extension is the chemotaxis pathway. The chemotaxis pathway is the reason why cells move in an environment. A cell is not stationary; it is a living organism that is constantly moving around in its environment (Figure 1.1). This motion is known as chemotaxis. The precise functionality of chemotaxis is further described in Chapter 2.3 .

The secondary focus was to create a multiscale interactive visual. This multiscale aspect can be thought of as a magnification of the cell from what we see with our naked eye down to its molecular level. By using a multi scale approach, we can observe not only the general behaviour of chemotaxis at the population level in an environment, but also navigate down to the cellular level to observe the processes in the individual cells that affects chemotaxis (Figure 0.1).

\subsection{Objectives}

Two objectives were planned and implemented for this project. As mentioned in the previous section, the foremost objective was the implementation of the chemotaxis pathway into our existing model.

The other primary objective was to implement the multiscale aspect as seen in Figure 0.4. Other than our primary objectives, a set of features were also implemented: 


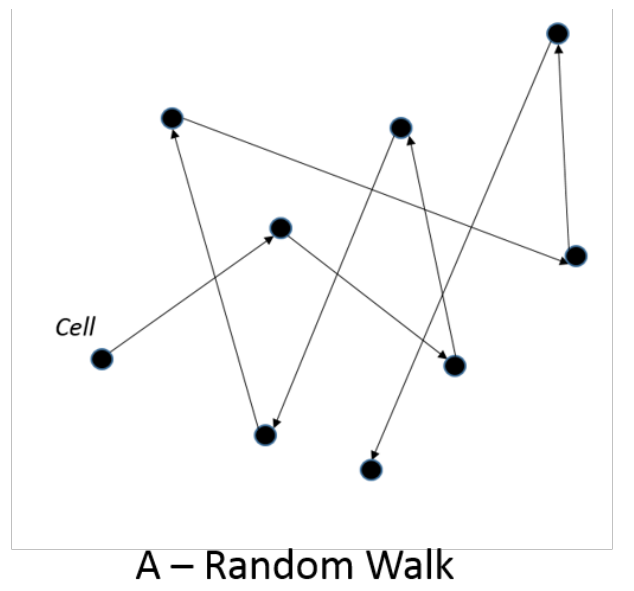

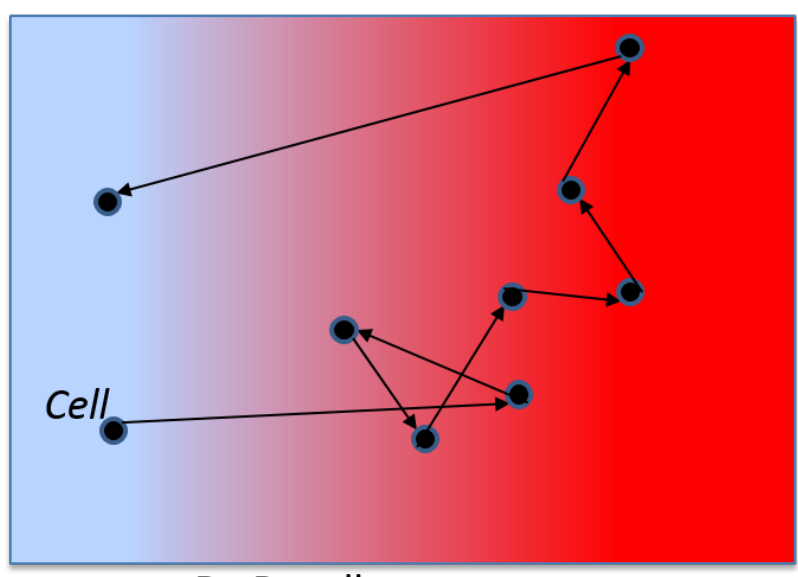

B - Repellent

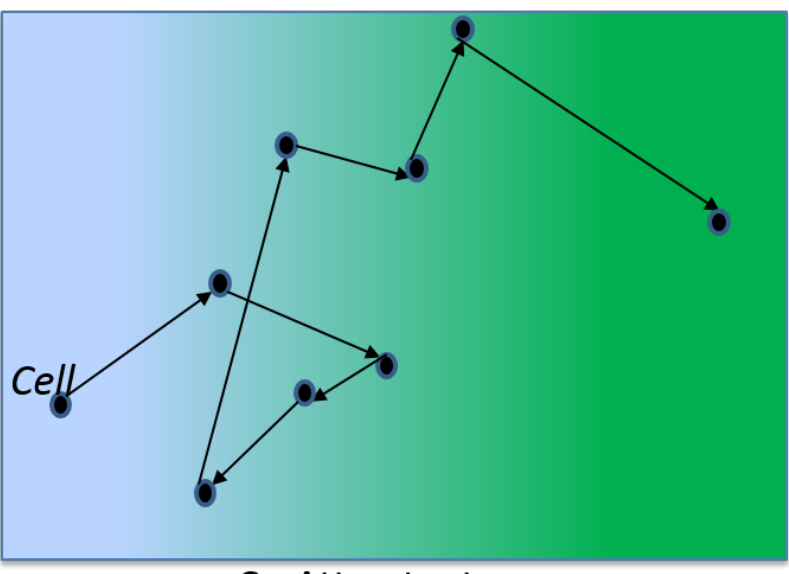

C - Attractant

Figure 1.1: A) Bacterium cell in a saturated or non-stimulus environment (Random Walk). B) Bacterium cell in an environment where the repellent stimulus is stronger on the right side (Represented by gradual increase in red colour alpha value from left to right), C) Bacterium cell in environment where attractant stimulus exists and comes from the right side (Represented by the gradual decrease in green colour alpha value from right to left). 
1. Interactive - The model will allow for interaction with the user in real-time.

2. Visual - The model will present the biological pathway using three-dimensional (3D) rendered graphics in our lab's simulation environment LINDSAY Composer 2.0.

3. Biologically Accurate - The model will present a biologically accurate representation of the biological pathway.

4. Robust - The model will allow for dynamic changes that will be reflected in real-time in the simulation.

The objectives and the feature's mentioned above have been addressed and are described throughout this thesis. The thesis is composed of eight chapters and three appendices. In each chapter, we explore a component that contributes greatly to the successful creation and implementation of our multiscale model.

Chapter 2 explores the biology behind our model, the model organisim chosen for this work and a detailed breakdown of the biological background of the chemotaxis pathway. Next in Chapter 3, we dive into the exploration of the fundamental works that contributed to our model. Previous models of chemotaxis are explored and the two modeling methods, the combination of the two modeling methods, and multiscale modeling are discussed.

In Chapter 4, we introduce the model's architecture and the implementation of each of the layers in the model, and the hybrid implementation of the two modeling methods. Next, in Chapter 5, the interactive ability of the model is discussed, with results presented in Chapter 6 .

The thesis concludes with a summary in Chapter 7 and future work being discussed in Chapter 8 .

Appendix A presents the simulation environment Lindsay Composer (LC). Appendix B describes the rendering and visualization techniques, tools and engines utilized in the 
implementation of our multiscale model. Appendix C describes the process of importing three-dimensional (3D) models into our model simulation.

\subsubsection{Milestones}

Throughout the development of this model, several challenges were encountered (discussed in Chapter 4.3. Each milestone presented a set of obstacles, and each of these obstacles were broken down. In this section, I will mention the milestones that were set, and eventually achieved.

\section{Milestone 1}

The first milestone was to learn, understand and use the simulation tool that our model is built upon. This tool is known as the LINDSAY platform version 1.0 [34]. With the support from our research lab, documentation and small projects, this first milestone was achieved. Esmaeili's model was built on version 1.0 of the LINDSAY platform, and his model and code was a very valuable resource in learning this tool.

\section{Milestone 2}

The next milestone that we had to achieve was to convert Esmaeili's model from version 1.0 of the LINDSAY platform to version 2.0. In version 2.0, the LINDSAY platform was optimized to handle data access more efficiently. Through countless hours of reading and testing, the conversion was eventually accomplished. The support from our research lab was crucial for the completion of this milestone.

\section{Milestone 3}

The third milestone was learning and understanding the biological pathway and process of chemotaxis in E. coli. With 40 years of research and studies, 
the information available is enormous, but as will be discussed in Chapter 4.3 , this amount of information served both as something good and bad.

\section{Milestone 4}

Technical implementation of the chemotaxis pathway and its cellular process involved the creation of four distinct simulations, one for each of the views (Figure 0.4). We have implemented a model of a colony of bacterial cells, which we can visualize and interact with at distinct, yet computationally interconnected levels.

\section{Milestone 5}

The final milestone was bringing together the implementation of the chemotaxis pathway on each of the proposed layers in the model to create a single multiscale model of chemotaxis.

\subsection{Additional Components Added to The Original Model}

In this section, the additional components added on top of the original model will be presented. Technical specifications will be discussed in Chapter 4 . This section will summarize my contributions to the overall model.

My first contribution is the addition of a chemotaxis pathway model. With the addition of the chemotaxis pathway, the simulated $E$. coli now has the ability to move throughout its environment.

The multiscale is the next major addition to the model. Unlike the previous model, this updated version allows us to transition between four layers of detail (Figure 0.1).

1. Naked-eye (naked-eye view) - $1^{\text {st }}$ layer

2. Microscopic (colony view) $-2^{\text {nd }}$ layer 
3. Individual cell (individual view) - $3^{\text {rd }}$ layer

4. Molecular (molecular view) $-4^{\text {th }}$ layer

Several interaction components (Chapter 5) have been added to allow the user to interact with the chemotactic pathway in the bacterium. Each layer is connected to an interface that acts as the controller for interaction in each layer (Figure 1.2). Each of the components are able to dynamically affect the cell in real-time in the simulation.

The time in our simulation can be controlled as well. As we can control the time in our simulation, the iteration time between time steps is a relative time rather than an exact time. Throughout the thesis, a time step $(\mathrm{T})$ is used as a sequential measurement of some relative time after the start of the simulation. Such that, time step $0(\mathrm{~T}=0)$ is the start of the simulation, and then time step $1(\mathrm{~T}=1)$ denotes a relative time after time step 0 .

The next important addition is the the data model component, which is a centralized module that brings together information to share between the layers. During the transition to another layer, the layer can load data from this module and process the retrieved data before the layer is rendered on the screen. This centralization of the data allows the layers to communicate and synchronize information. 


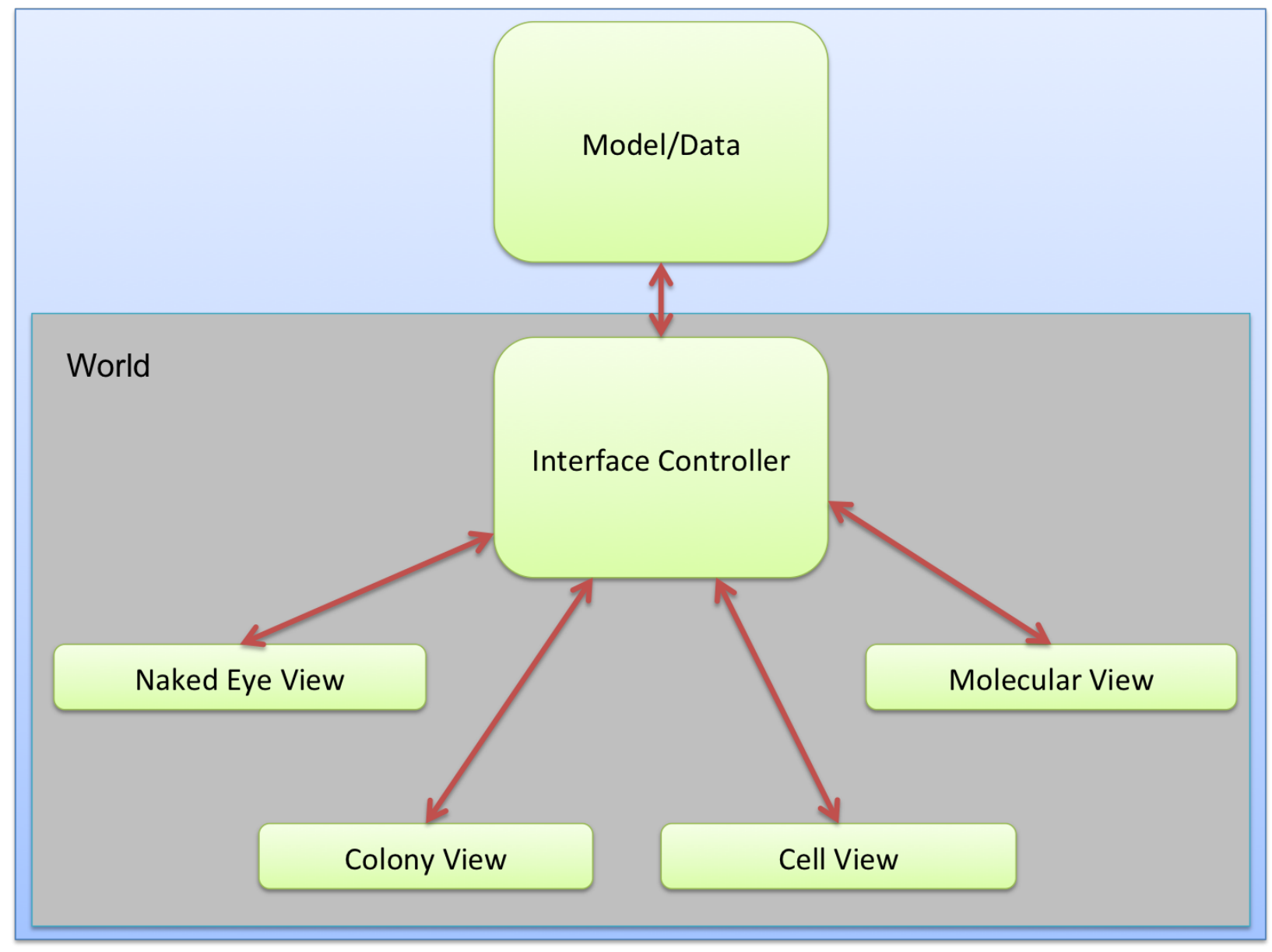

Figure 1.2: Architecture of the connections between each of the layers, the interface and the data behind each of the layers. 


\section{Chapter 2}

\section{BIOLOGY: Escherichia coli and Chemotaxis}

The prokaryotic cell we modelled is known as Escherichia coli (E. coli ). E. coli is used for this model because it is a significantly researched organism in Biology, Moreover, it is the organism used in the model that served as the starting point for our model [27]. The popularity of $E$. coli in research is due to its single cell composition and ease of manipulation in different environments [13. In the following sections, E. coli and the chemotaxis pathway will be further discussed.

\subsection{Escherichia coli}

E. coli was first discovered in 1885 by Theodor Escherich, a German bacteriologist [28]. It was first named as Bacterium coli, but was renamed Escherichia coli to honour Escherich's contributions to the study of this bacterium. Most strains of E. coli are harmless as we have billions naturally residing within our intestinal tracts [64]. However, Escherich's research discovered that certain strains of E. coli cause diarrhea and gastroenteritis [28].

E. coli has been the centre of many biological discoveries due to its ease of growth and adaptability to different conditions and manipulation of its genome [13]. The genome of $E$. coli encodes for 4300 proteins, a relatively small number compared to other organisms [64]. It is also one of the first organisms to have its entire DNA sequenced [64]. The above points make $E$. coli a relatively easy organism to work with, making it one of the most researched organism in the world. 


\subsection{Chemotaxis}

"Chemotaxis is the biasing of movement towards regions that contain higher concentrations of beneficial, or lower concentrations of toxic chemicals." [62]

Chemotaxis was first discovered in 1880 by Theodor Wilhelm Englemann [13]. It is a universal attribute of motile cells and organisms, and dictates the movement of the cell or organism in the presence of a stimulus. This movement of the organism towards favorable conditions is shown in Figure 1.1. Chemotaxis is a vital factor for the survival of an organism.

The stimulus - often a chemical (hence "chemo") - is either an attractant (positive for the organism), or a repellent (negative for the organism). These attractants and repellents are commonly referred to as chemo-attractants or chemo-repellents [13]. In the case of a chemoattractant, the organism will swim towards a high gradient area of the source (Figure 2.1), thereby exhibiting a positive chemotactic response. Correspondingly, chemo-repellents will cause the organism to flee and thereby exhibiting a negative chemotactic response (Figure 2.2 .

The context of a stimulus in this thesis refers to the sudden addition or change of chemoattractant or repellent in the bacterium's environment. It is assumed that before the addition of the stimulus, its environment either contains no chemo-attractants or repellents or the environment is already at saturation.

\subsubsection{Chemotaxis and E. coli}

Chemotaxis has been studied in E. coli for over 40 years [13]. E. coli is a motile bacterium and chemotaxis is important for its survival. Similar to any other motile cell, in the presence of chemo-attractants $E$. coli will move towards the higher concentration area of the source and conversely with chemo-repellents, E. coli will move towards a lower concentration area. Because E. coli cells are constantly moving and replicating, the colony of cells expands in 

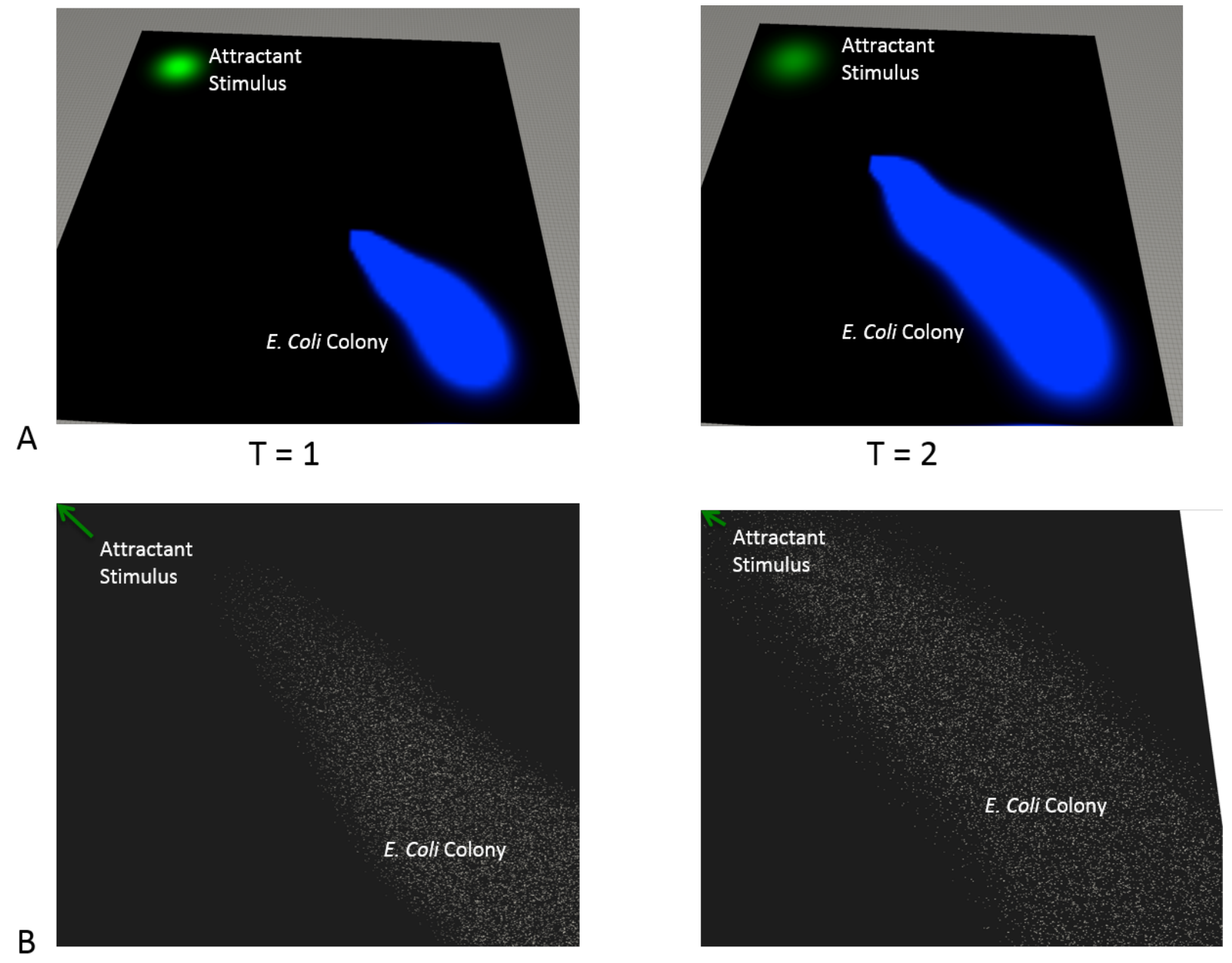

Figure 2.1: Screen captures of chemotactic movement toward an attractant stimulus shown at time step $1(\mathrm{~T}=1)$, and time step $2(\mathrm{~T}=2)$ during the simulation. A) Gradient visualization under the naked-eye view (layer one), B) Zoomed in colony view (layer two) of the same scene. 

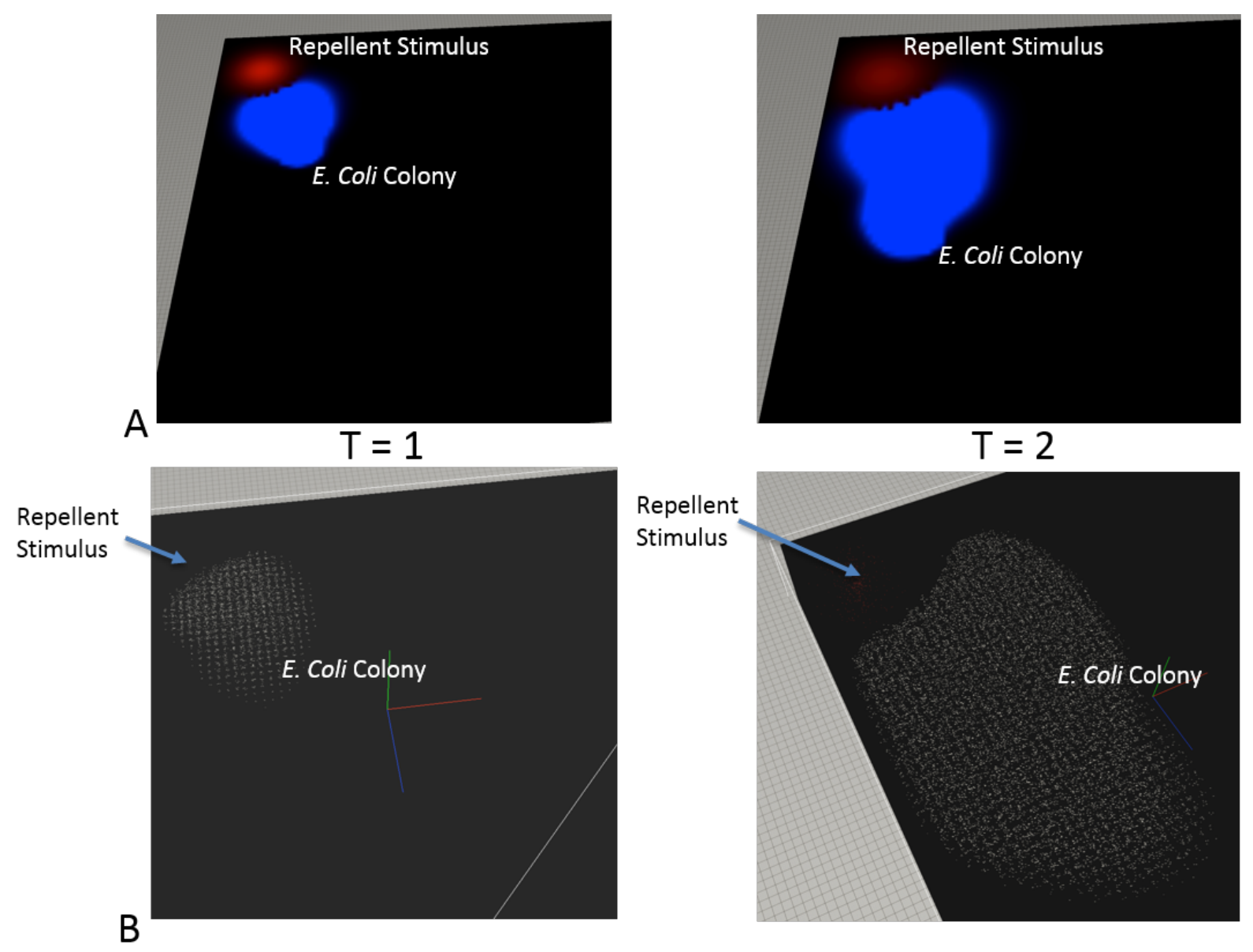

Figure 2.2: Screen captures of chemotactic movement away from a repellent stimulus shown at time step $1(\mathrm{~T}=1)$, and time step $2(\mathrm{~T}=2)$ during the simulation. A) Gradient visualization under the naked-eye view (layer one), B) Zoomed in colony view (layer two) of the same scene. 
elliptical shapes known as chemotactic rings as Figure 2.3 demonstrates. Depending on the stimulus, the preferred position of the bacterium is apparent as Figure 2.1 and Figure 2.2 shows.

Since the discovery of chemotaxis, no significant progress was made in chemotaxis research until the 1960s by Julius Adler [13]. Adler reported that specific receptors in bacteria are used to recognize the chemical stimulants in the environment [2, 3, 4, 5, 6]. There are 5 different types of receptors known as the methyl-accepting chemotaxis proteins (MCPs): Tsr, Tar, Tap, Trg, and Aer. Each of the MCPs detects different chemicals and these MCPs are usually bundled into clusters at the poles of the bacterium [4, 56]. For simplicity, in our model we include one generic receptor which subsumes all MCP properties, such that this one receptor will detect chemicals that can be detected by the MCPs. Adler's work was a significant leap in chemotaxis research.

\subsection{Chemotaxis in E. coli}

The pathway of chemotaxis in E. coli is composed of two distinct networks [33]. The two responses that drive the cellular process of chemotaxis are known as the Signal Transduction Cascade (Excitation) and Methylation (Adaptation) [13, 33, 62. Together, the responses control the chemotactic behaviour of $E$. coli . This chemotactic behaviour is a series of runs and tumbles the bacterium performs during its life-span. The movement of the bacterium is propelled by flagella filaments as seen in Figure 2.4 that extend from the cellular membrane [7]. Each flagella filament is controlled at its base by a motor inside the cell membrane [33] as shown in Figure 2.6. The rotation of the flagella will determine whether the bacterium runs or tumbles as shown in Figure 2.5.

A "run" is defined by the bacterium swimming in a forward motion. This forward motion

\footnotetext{
${ }^{1}$ Printed with permission from Dr. John S. Parkinson Lab http://chemotaxis.biology.utah.edu/ Parkinson_Lab/projects/ecolichemotaxis/ecolichemotaxis.html
} 


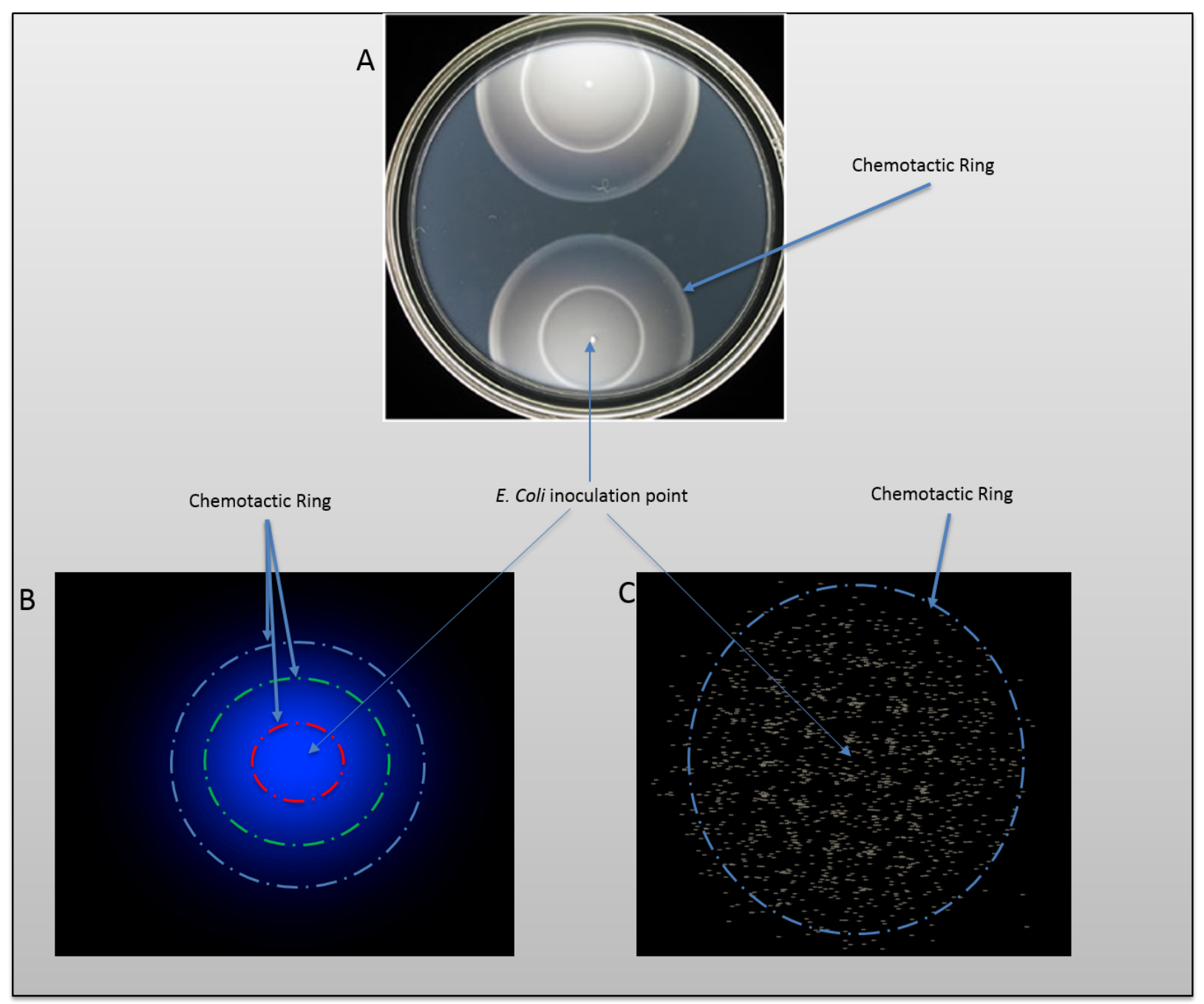

Figure 2.3: A) A tryptone soft agar plate in which motile cells can swim through waterfilled tunnels in the agar. Two chemotactic colonies are shown. As the cells grow, they establish attractant gradients through consumption energy sources saturated in the agar 1 . B) Representation of chemotactic ring in layer one of our multiscale model (naked-eye view). C) Representation of chemotactic ring in layer two of our multiscale model (colony view). 


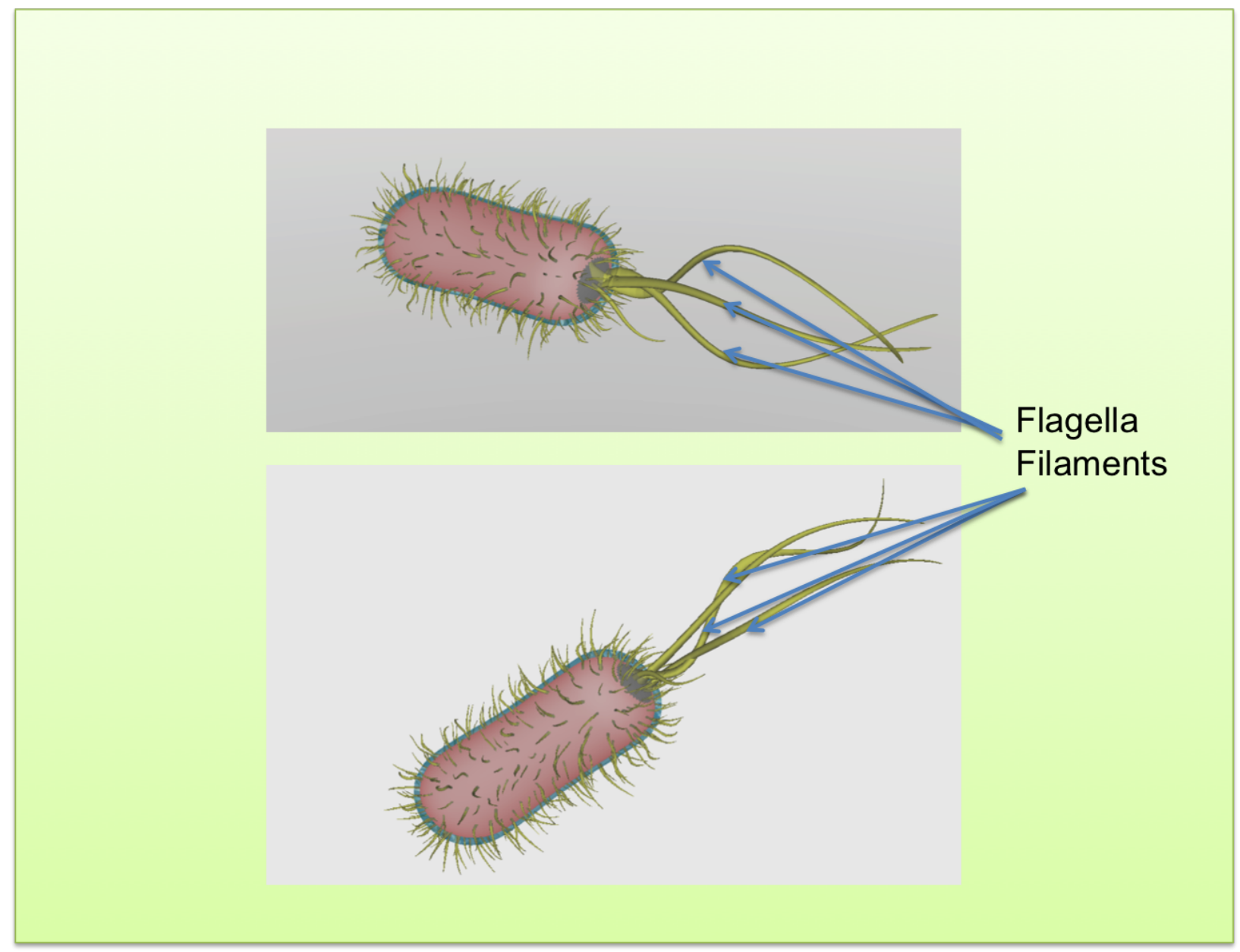

Figure 2.4: Flagella filaments of the E. coli bacterium. 


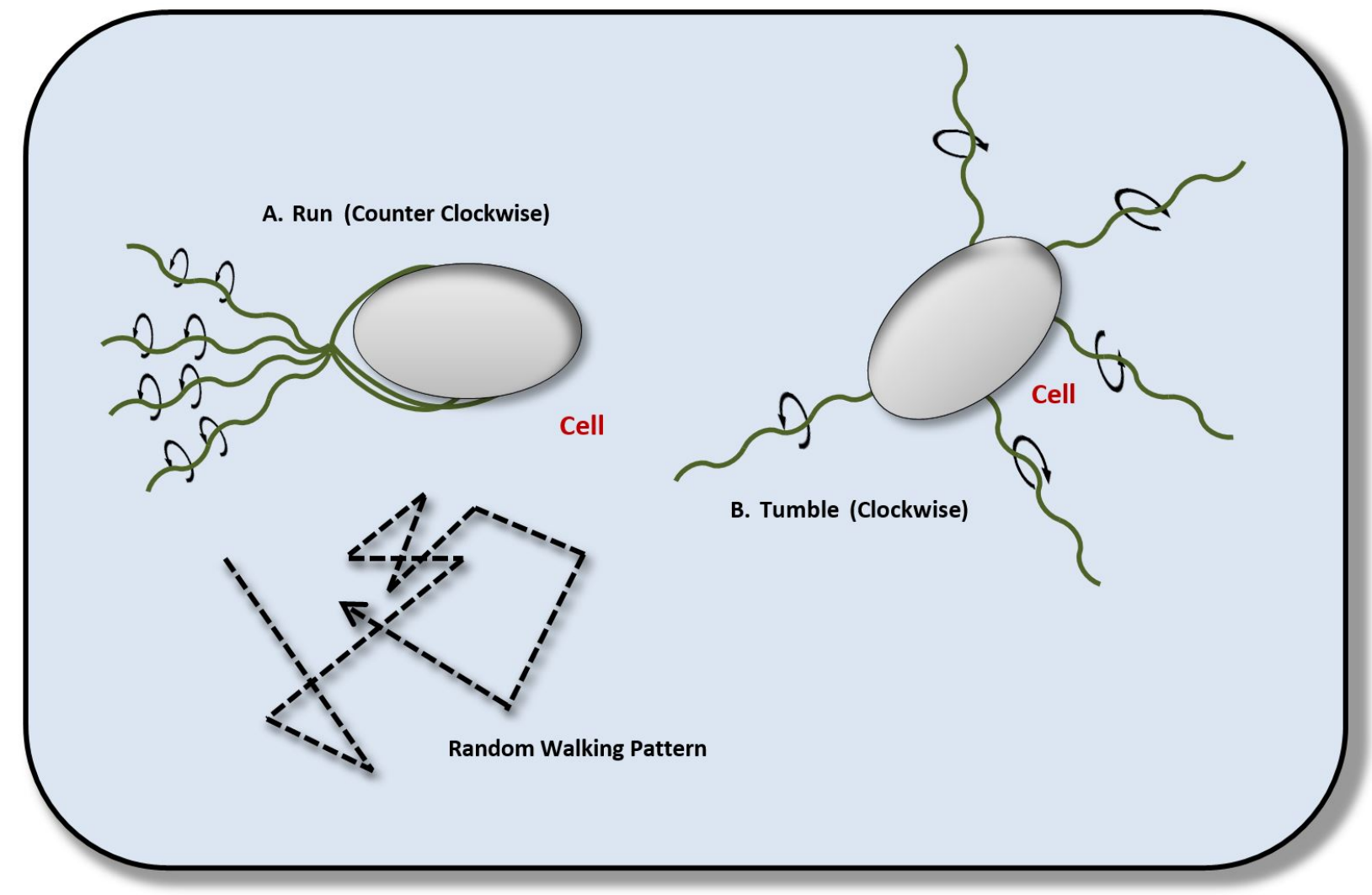

Figure 2.5: A) The flagella filaments rotate CCW, they bundle together and propel the bacterium forward. B) The flagella filaments rotate $\mathrm{CW}$, breaking the bundle and causing the bacterium to tumble.

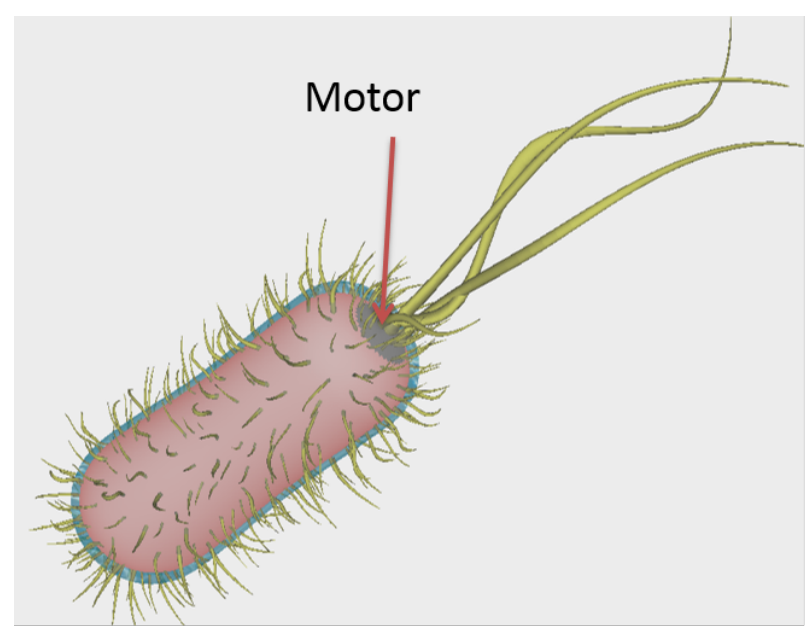

A

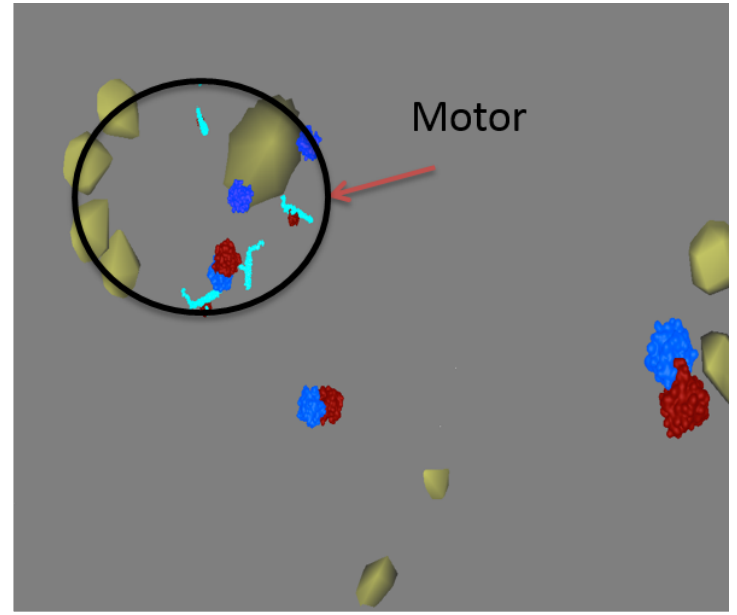

B

Figure 2.6: A) The Motor location from outside the E. coli bacterium. B) The Motor location from inside the $E$. coli bacterium. 
occurs when the flagella on the bacterium rotate counter clockwise (CCW) [13]. During this rotation, the flagella bundle up together to propel the bacterium forward as demonstrated by Figure 2.5A.

A "tumble" is defined by the bacterium changing its direction of travel. During the tumble phase, the flagella rotate clockwise (CW) [13]. This rotation causes the flagella to break from the bundle, which results in the loss of forward propulsion leading to a tumble (Figure 2.5B).

In the absence of a stimulus, the runs and tumbles of the bacterium are balanced. There is no defined location where the bacterium should travel towards, this is known as random walk as seen in Figure 1.1A. In the case of random walk, there is no external disturbance to bias the rotations of the flagella [7]; hence, as shown in Figure 2.3, the E. coli growth and movement is circular rather than elliptical.

In the presence of a stimulus - whether it be an attractant or repellent - the rotation bias of the flagella is affected by it. Molecules of the stimulus are picked up by the receptors protruding out of the cell membrane. This stimulus then triggers the Signal Transduction Cascade in the chemotaxis pathway which inadvertently also affects the Methylation response in the pathway.

With or without a stimulus, E. coli is constantly comparing the concentration of its current location to its previous location. This concept which is reported by Segall et al. [54 implies that the concentration detected by the E. coli is temporal in that it possesses a memory that compares present and past information.

The proteins involved in chemotaxis are summarized in Table 2.1. Each protein plays a specific role in one of the two responses in the chemotaxis pathway. 


\begin{tabular}{l|r}
\hline Protein & Role \\
\hline Chemotaxis Protein A (CheA) & Phosphorylation protein \\
\hline Chemotaxis Protein B (CheB) & Demethylation protein \\
\hline Chemotaxis Protein Y (CheY) & Signal transmission protein \\
\hline Chemotaxis Protein Z (CheZ) & Dephosphorylation protein \\
\hline Chemotaxis Protein R (CheR) & Methylation protein \\
\hline Chemotaxis Protein W (CheW) & Binds with CheA to form CheAW complex \\
\hline
\end{tabular}

Table 2.1: Chemotaxis Proteins and Their Roles in the Pathway.

\subsubsection{Excitation Response - Signal Transduction Cascade}

Signal transduction or the excitation response is responsible for directly affecting the motion of the bacterium. In this response, a series of signals are transferred downstream from the receptor to the motor of the flagella.

The receptor's activity varies depending on the current biochemical reactions happening inside the cell [7]. To keep our model simple, we assume that receptors can only be active or inactive. This corresponds to whether they activate the CheA and CheW complex downstream from the receptor [47]. We will refer to the complex made of CheA and CheW as CheAW. The complex CheAW is bound to the end of the receptor inside the cell membrane. The receptor's inactive state corresponds to an increasing attractant signal [47], such that with an increase in attractant concentration the receptor becomes inactive and thereby suppressing the tumbling of the bacterium.

Figure 2.7A.1 is a schematic representation of the Signal Transduction Cascade which is described here. CheAW's state is dependent on the receptor. An active state would result in phosphorylation of $\mathrm{CheY}$ and $\mathrm{CheB}$ (Figure 2.7A.1, and 2.8A.1). CheB's role will be further discussed in chapter 2.3.2. CheZ's role is to dephosphorylate CheY-P to return it to the unphosphorylated state CheY. The rotation of the flagella is dependent on the concentrations of CheY and CheY-P. A CCW rotation results if the CheY concentration is greater. Likewise, CW rotation occurs if the CheY-P concentration is greater.

Increasing attractant or decreasing the repellent concentration will bias the bacterium 
into swimming in smooth arcs by suppressing CheY phosphorylation and thereby increasing the CheY concentration. Similarly, an increase in repellent or decrease in attractant concentration results in an increase of CheY-P leading to an increase in tumbling to change the direction of the cell.

\subsubsection{Adaptation Response - Methylation}

Adaptation is the process of the cell returning to its normal state of behaviour. In the normal state, the cell again searches for a favorable direction to travel in. Without methylation, the cell would continuously travel in a straight line regardless of the conditions. Methylation ensures that there is a recovery condition in the cell so that it may continue to query the temporal environment around itself for a better location to travel towards.

Methylation involves again the CheA-CheW complex (CheAW) and also CheR and CheB. When CheB is phosphorylated by CheAW, it is able to use its phosphatase group to demethylate the receptor [13, 6]. After demethylation, phosphorylated CheB (CheB-P) returns to its normal state CheB. Regardless of conditions inside or outside the cell, CheR will constantly methylate the receptor. Recall that the receptor has two states. In its active state, the receptor is methylated and in its inactive state, the receptor is demethylated. The adaptation response is to ensure that the receptor can be active again after a stimulus molecule is bound to the receptor. Refer to Figure 2.8A.1 for a schematic representation of the Adaptation process.

\subsubsection{Negative Feedback Loop - Adaptation}

Negative feedback occurs when the result of a change in the process also results in a gradual return to an equilibrium state [7], or the state the process was in before a change occurred. This is precisely how adaptation in chemotaxis functions. This process is responsible for the ability of the bacterium to perform a series of tumbles and runs throughout its life- 
span. Negative feedback is responsible for the robustness property of a system [7] and it is a stabilizing factor in the system. Robustness allows the system to cope with external disturbances [7]. In the case of chemotaxis, the disturbances would be the stimulus in the environment.

The process of negative feedback is directly correlated to CheB since CheB is responsible for demethylation and demethylation is proportional to the activity of CheAW [52]. The activity of CheAW is directly related to the activity of the receptor [52].

To understand how the negative feedback mechanism works, the excitation and adaptation response must be looked at together. Recall that during the excitation response, the receptor is inactivated which inactivates the CheAW complex, and the adaptation is the recovery of the inactive receptor back to its active state. Keep in mind that an active receptor is a methylated receptor and in order for the receptor to be reactivated, the methylation must occur faster than the demethylation process.

This is where the negative feedback loop occurs, CheB-P demethylates the receptor, but CheB-P exists only if CheB is phosphorylated by CheAW. This can only occur if CheAW is active. In Chapter 2.3.1, we mentioned that the binding of a stimulus molecule to the receptor inactivates the receptor, which inactivates CheAW. Phosphorylation does not occur when CheAW is inactive. This leads to a decrease in CheB-P concentration, which results in a faster methylation process than demethylation. This results in the reactivation of the receptor, and thereby reactivating CheAW. Consequently, CheB-P and CheR alternate in deactivating and reactivating the receptors (Figure 2.8).

Now that we know about the biology of chemotaxis, we can proceed to the model. In the following Chapter we will look at various models developed over a span of half a century that describe the biological process of chemotaxis. 


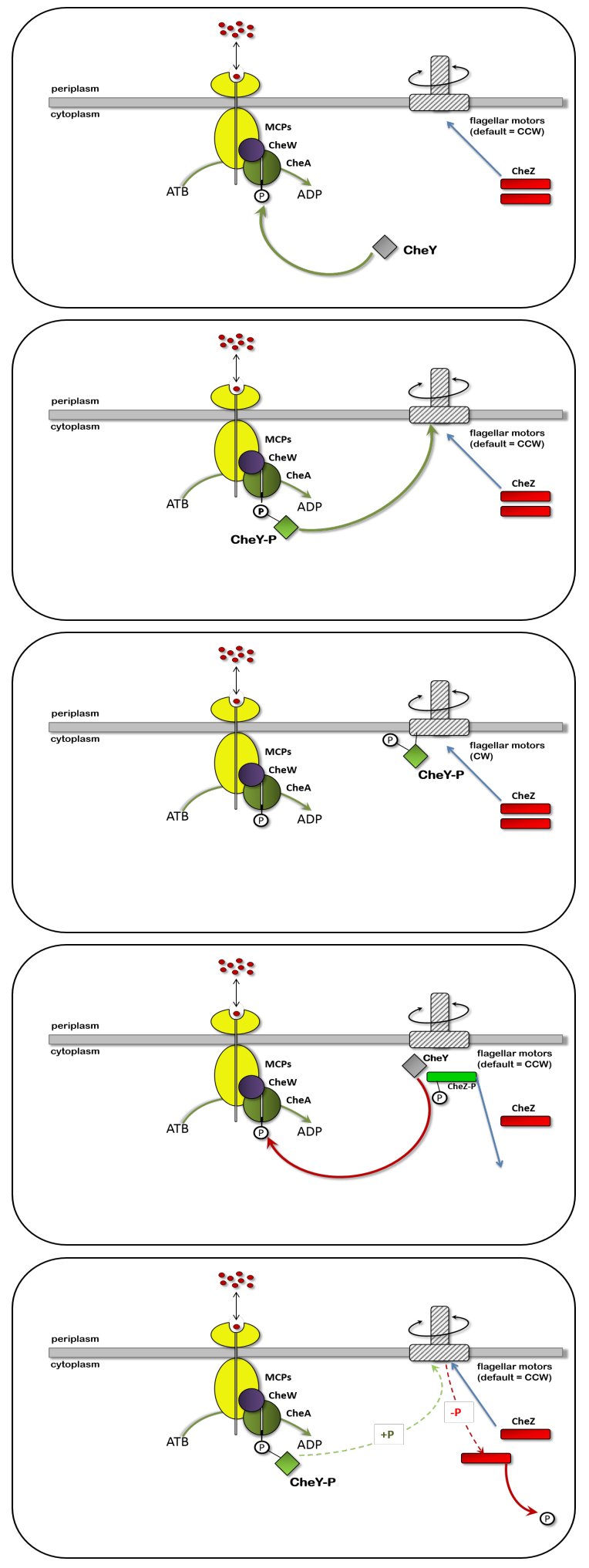

A.1
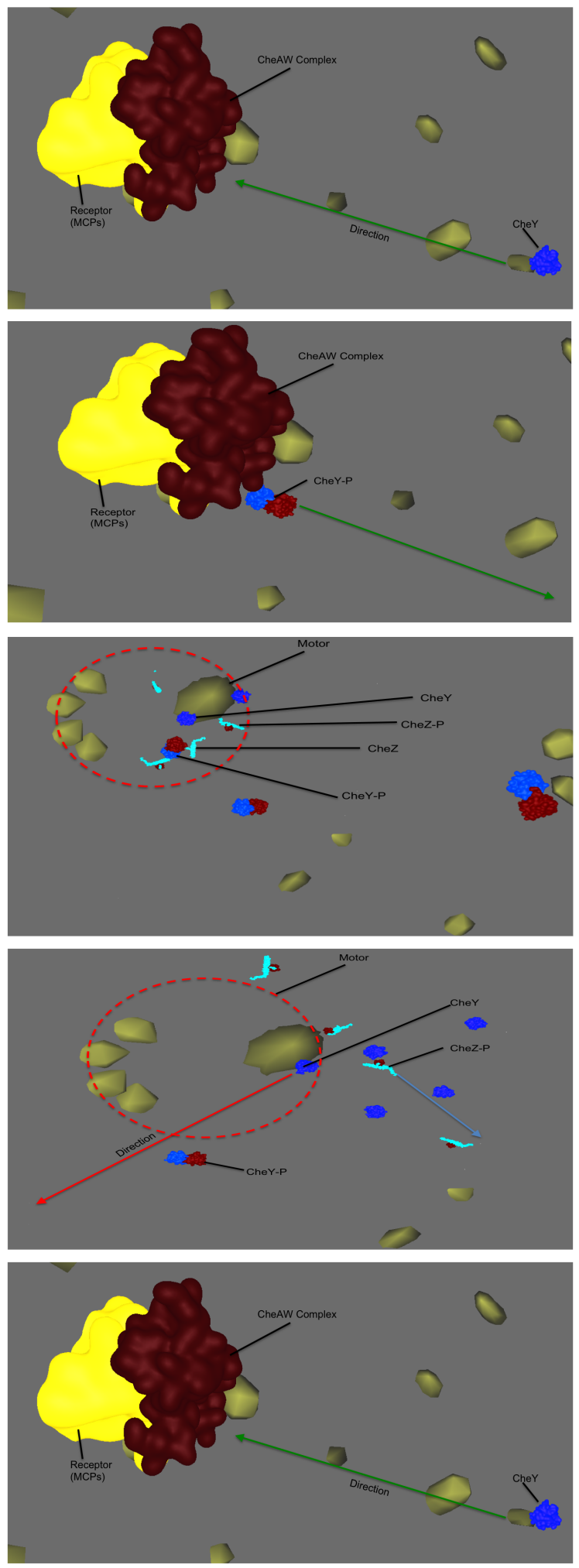

A.2

Figure 2.7: Chemotactic Pathways: schematics and translations into our agent-based E. coli model. A.1) Excitation. A.2) Agent interactions for excitation. 


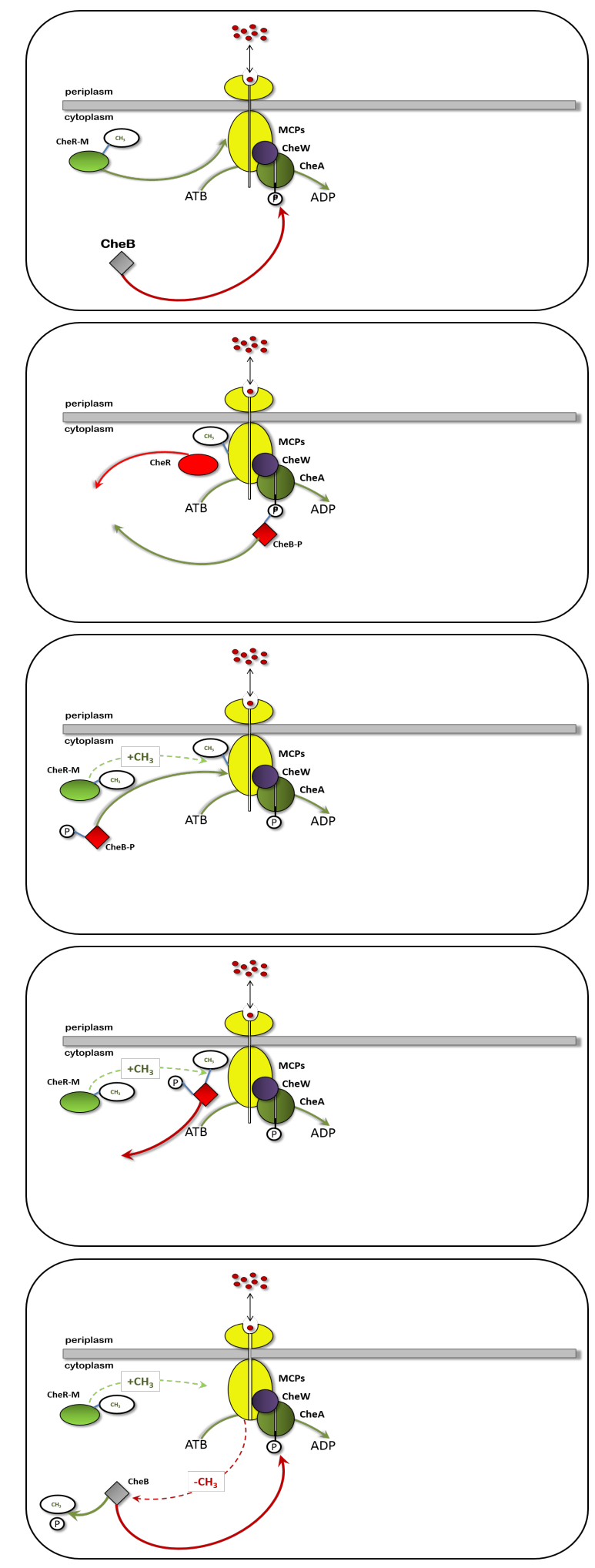

A.1
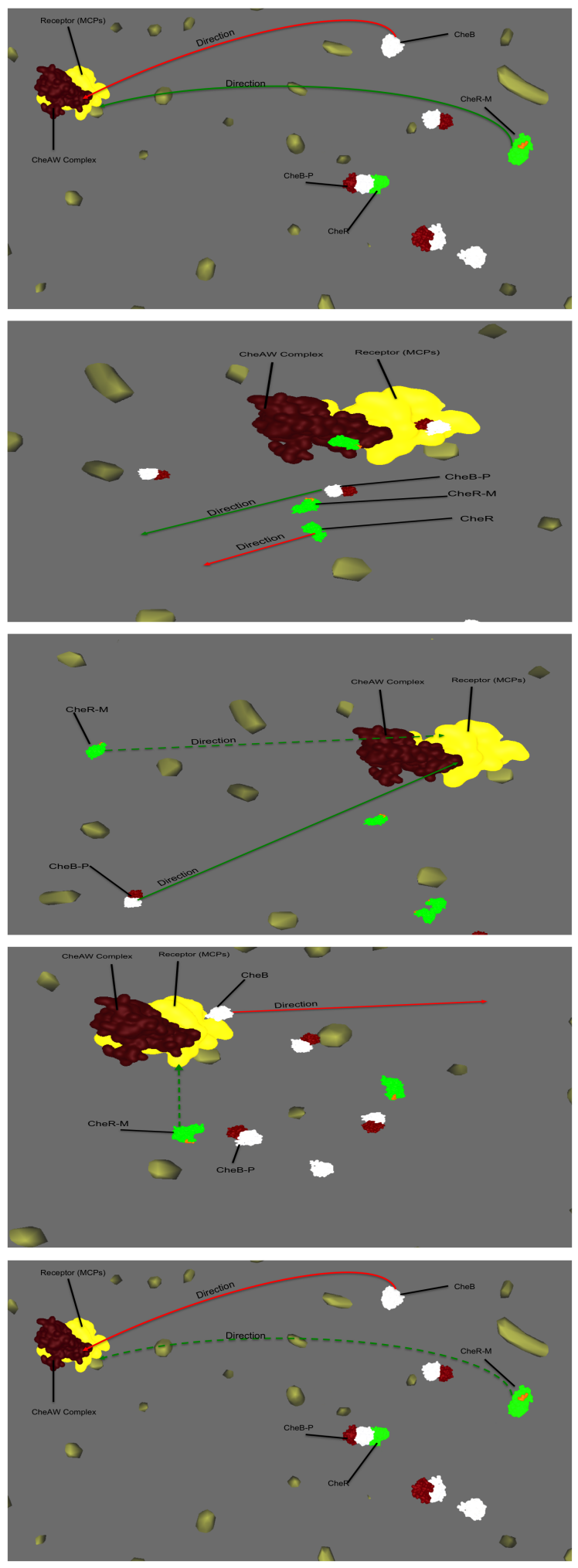

A.2

Figure 2.8: Chemotactic Pathways: schematics and translations into our agent-based E. coli model. A.1) Adaptation. A.2) Agent dynamics during adaptation response. 


\section{Chapter 3}

\section{Chemotaxis Models}

Modelling systems involves one of two conventional methods: mathematical or agent-based. The method of choice is dependent on the system or process being modelled. In recent years, a new method known as hybrid modelling is being utilized to model complex systems. This method is precisely what we are using to create our chemotaxis multiscale model. This method combines both the mathematical and agent-based methodologies and switches between these two techniques as required during the simulation of the model. Each of these methods and its relevant work pertaining to chemotaxis will be explored in this chapter.

\subsection{Previous Models of Chemotaxis}

Over the years, much research into E. coli and chemotaxis have generated several models as shown in Table 3.1. These models are vital in contributing to the understanding of chemotaxis. It is important to note that every model developed expands upon a previous model. Although all these models are of chemotaxis, they differ in their comprehensiveness and coverage of the pathway [29]. Some models cover the general principles while others cover a particular part in the pathway. They also differ in the depth of details covered. [29]

Chemotaxis models usually fall into one of two categories: fine-tuned or robust. In a fine-tuned model, the biochemical parameters must be precise. The model itself depends on these parameters to be accurate [7]. The robust model on the other hand is able to deal with a wide range of parameters [7].

Keller and Segel [35] in 1970-71 proposed a mathematical model that was originally developed to model and analyze the movement of slime molds. However, the model they developed now serves as the foundation for modelling chemotaxis at the population level. 
This model can be found as the basis in nearly every model developed for chemotaxis at the population level.

In 1986, Albert Goldbeter, Lee Segel, and colleagues [36, 7] proposed a theoretical model for the adaptation process. This theoretical model formed the important basis for later theoretical work on the adaptation response. Goldbeter and Segels' model is often found as the foundation block in fine-tuned models where the parameter is based on a specific value.

The first attempt at a computer model was by Bray et al. in 1993 [20]. They developed the Bacterial Chemotaxis Program (BCT), and the first version only modelled the excitation response. In 1995, an updated version of the BCT was released, it still only modelled the excitation response, but incorporated a more biologically accurate representation of the receptor complexes [19], and the binding affinities were optimized by an evolutionary algorithm [29]. Bray et al. [21] continued to evolve their model, in 1998 receptor clustering and sensitivity was improved. In 2002, Bray et al. attempted to incorporate the amplification process of the receptors into the model. Since then, there have not been updates on BCT as focus has shifted towards StochSim, an improved version of BCT that includes stochastic features.

In 1997, Barkai and Leibler [12] worked on a model for the adaptation response in chemotaxis. Their proposed model differed from previous models in the way that it was not a finetuned model [7]. Barkai and Leibler's model took into account a wide range of possible values for biochemical parameters rather than just one specific value. Their model included several methylation sites and reproduces many observations of the dynamic behaviour of chemotaxis [12. Barkai and Leibler, using a three-component model showed that the adaptation process is robust rather than fine-tuned [7].

In 1999, Morton-Firth et al. [49], along with the BCT team, developed the first stochastic simulation model of bacterial chemotaxis. This program became known as StochSim, an improved version of the BCT program. StochSim was able to incorporate both the excitation 
and adaptation responses into its model. From 2001 to 2003, Steve Andrews working with the original BCT team, developed the Smoldyn [9] program. Smoldyn simulates cell-scale biochemical reactions to capture natural stochasticity data. The program was developed to provide a more realistic way to simulate the diffusion of signalling molecules through the cytoplasm [9].

Following in 2005, Emonet et al. developed the first known multiscale model of chemotaxis [26]. Utilizing StochSim as its core engine, Emonet et al. developed AgentCell. AgentCell utilized concepts of agent-based modelling to represent chemotactic responses at the population and single cell level. AgentCell was able to accurately produce validated results under both stimulated and unstimulated conditions.

Bray et al. developed E. solo [23] in 2007, a model that uses ordinary differential equations to model the signalling reactions in the pathway. E. solo then provided a graphical display of the bacterial movement in a two-dimensional environment.

Table 3.1 summarizes the models developed in chronological order and their emphasis on the chemotactic pathway.

\begin{tabular}{l|l|l}
\hline Model & Summary & Emphasis \\
\hline Keller and Segel [35] & $\begin{array}{l}\text { Model proposed to describe the chemo- } \\
\text { tactic response at the population level. }\end{array}$ & Chemotactic Motion \\
\hline Knox, Goldbeter, Segel [36] & $\begin{array}{l}\text { Proposed theoretical model on the } \\
\text { adaptation response of the Chemotaxis } \\
\text { Pathway. }\end{array}$ & Adaptation \\
\hline Bray et al. 20] & $\begin{array}{l}\text { First attempt at a computer model, } \\
\text { known as the BCT model. The Excita- } \\
\text { tion response is simulated, but not the } \\
\text { methylation adaption process. CheR } \\
\text { and CheB are present but are not ac- } \\
\text { tively used. }\end{array}$ & \\
\hline
\end{tabular}




\begin{tabular}{|c|c|c|}
\hline Bray and Bourret 26] & $\begin{array}{l}\text { Version } 2 \text { of the BCT program. It } \\
\text { incorporates a more biologically ac- } \\
\text { curate model of the receptor com- } \\
\text { plex (CheA/CheW ligand) and bind- } \\
\text { ing affinities of the receptor optimized } \\
\text { by an evolutionary algorithm. }\end{array}$ & Chemoreceptor Formation \\
\hline Hauri and Ross 33. & $\begin{array}{l}\text { Parameters used in model based on } \\
\text { experimental measurements, however } \\
\text { they are adjusted (fine-tuned). This } \\
\text { was the first model to simulate exact } \\
\text { adaptation in both attractant and a re- } \\
\text { pellent stimuli. }\end{array}$ & Excitation and Adaptation \\
\hline Barkai and Leibler 12$]$ & $\begin{array}{l}\text { Model was based on robustness. The } \\
\text { model was a three-component model } \\
\text { showing that adaptation is a robust } \\
\text { property and that adaptation time is } \\
\text { inversely proportional to receptor ac- } \\
\text { tivity. }\end{array}$ & Robustness and Adaptation \\
\hline Spiro et al. 57 & $\begin{array}{l}\text { Simplified three-component model, ad- } \\
\text { justed by trial and error to simulate } \\
\text { the response to temporal variation of } \\
\text { aspartate. }\end{array}$ & $\begin{array}{l}\text { Excitation, Adaptation and Receptor } \\
\text { Sensitivity }\end{array}$ \\
\hline Bray et al. 21] & $\begin{array}{l}\text { Receptor clustering and sensitivity al- } \\
\text { gorithms improved }\end{array}$ & Receptor Sensitivity \\
\hline Levin et al. 40 & $\begin{array}{l}\text { Methylation state reactions are incor- } \\
\text { porated into the BCT program. Also } \\
\text { known as the adaptation network in- } \\
\text { side the chemotaxis pathway. }\end{array}$ & Adaptation \\
\hline Morton-Firth et al. 49] & $\begin{array}{l}\text { First stochastic simulation of chemo- } \\
\text { taxis. Utilizing the StochSim program. } \\
\text { Incorporation of both ligand binding } \\
\text { and the methylation network. Simu- } \\
\text { lation looked into local vs global adap- } \\
\text { tation. }\end{array}$ & $\begin{array}{l}\text { Excitation, Adaptation, Stochastic } \\
\text { Simulation }\end{array}$ \\
\hline Bray et al. & $\begin{array}{l}\text { Attempted inclusion of amplification } \\
\text { phenomena in bacterial sensing }\end{array}$ & Receptor Sensitivity \\
\hline
\end{tabular}




\begin{tabular}{|c|c|c|}
\hline Shimizu et al. 55 & $\begin{array}{l}\text { Extension of StochSim allowing inter- } \\
\text { actions between neighbouring receptor } \\
\text { complexes. Looked in the analysis of } \\
\text { energy patterns in the pathway. }\end{array}$ & Receptor Clustering and Sensitivity \\
\hline Mello and Tu 44 & $\begin{array}{l}\text { Deterministic model of the StochSim. } \\
\text { Used to determine conditions under } \\
\text { which the system achieves perfect } \\
\text { adaptation. }\end{array}$ & Adaptation \\
\hline Lipkow et al. 41 & $\begin{array}{l}\text { Another Stochastic model of the signal } \\
\text { transduction network in chemotaxis } \\
\text { using the Smoldyn program. Simu- } \\
\text { lated the movement of CheY molecules } \\
\text { and their contact with the Flagella } \\
\text { complex. }\end{array}$ & Excitation \\
\hline Emonet et al. [26] & $\begin{array}{l}\text { Multiscale model implementation in } \\
\text { AgentCell, an agent-based program } \\
\text { that uses stochastic modelling. An ex- } \\
\text { tended add-on of the StochSim pro- } \\
\text { gram, the simulation reproduced mo- } \\
\text { tions of single cells and diffusion mo- } \\
\text { tions of populations under different } \\
\text { conditions. }\end{array}$ & Hybrid Modelling, Multiscale \\
\hline Bray et al. 22 & $\begin{array}{l}\text { E. Solo model which uses ordinary dif- } \\
\text { ferential equations (ODE) of the sig- } \\
\text { nalling reactions in the pathway and } \\
\text { gives a graphical display of the bacte- } \\
\text { rial motion in a } 2 \mathrm{D} \text { environment }\end{array}$ & Excitation \\
\hline
\end{tabular}

Table 3.1: A Chronological History of Chemotaxis Models and Their Emphasis

\subsection{Mathematical Models}

The mathematical method of modelling has enormous success in various other disciplines such as physics, astronomy, business, and engineering [27]. Modelling a biological system is a difficult task due to the high level of complexity and lack of quantitative information [27]. However, with advancements in experimental methods and technological improvements, 
mathematical modelling has become prominent in modelling biological systems [60].

Mathematical modelling of biological systems usually involves complicated mathematical equations. These equations fall into one of two categories: Ordinary Differential Equations (ODE) or Partial Differential Equations (PDE) 63]. The type of differential equation used is dependent on the biological process being modelled. The difference between ODE and PDE is their number of independent variables and derivatives [63]. Ordinary equations deal with functions containing one independent variable and its derivatives. In contrast, partial equations deal with functions that contain more than one independent variable.

Mathematical modelling of chemotaxis uses both types of differential equations depending on which part of the pathway the model is describing. One of the earliest mathematical models of chemotaxis is by Keller and Segel [35]. This mathematical model became known as the Keller-Segel model. Their model serves as the foundation block of all other models to be formulated in the later years. The Keller-Segel model considers both the bacteria and the chemotactic substrate in a continuum limit, which results in a coupled system involving two PDEs [30]. This model describes the density or growth rate of bacteria given a specific stimulus concentration in the environment.

$$
\begin{gathered}
\frac{\partial y}{\partial x}=D_{n} \frac{\partial^{2} n}{\partial x^{2}}-\frac{\partial}{\partial x}\left(n X(S) \frac{\partial S}{\partial x}\right) \\
\frac{\partial S}{\partial t}=D_{S} \frac{\partial^{2} S}{\partial x^{2}}-k(S) n
\end{gathered}
$$

The two PDEs in the Keller-Segel model solve for $n$ and $S$ respectively, where $n \equiv n(x, t)$ is the density of cells and $S \equiv s(x, t)$ is the concentration of the stimuli in the environment. $D_{n}$ and $D_{s}$ are diffusion constants of cells and of the stimuli. The chemotactic sensitivity $(X(S))$ controls the strength of chemotaxis which is dependent on the concentration of substrate $S$ which is consumed by the cells at rate $k(\mathrm{~S})$.

In 1997, Barkai and Leibler proposed a mathematical model using ODEs [12]. 


$$
\frac{\partial m}{\partial t}=\frac{k_{R} R T^{I}}{K_{R}+T^{I}}-\frac{k_{b} B_{p} T^{A}}{K_{B}+T^{A}}
$$

The original model proposed by Barkai and Leibler is a three-state model. However, a simplified version of it known as the two-state model is often used in modelling. This two-state model assumes that CheR $(R)$ binds only to inactive receptors $\left(T^{I}\right)$ and that phosphorylated CheB $\left(B_{p}\right)$ binds only to active receptors $\left(T^{A}\right)$. In equation $(3.3)$, we can see a simplified version of the model that describes receptor activity $(m)$. The symbols $k_{B}$ and $k_{R}$ are the rate constants and $K_{B}$ and $K_{R}$ are the Michaelis constants for receptor demethylation and methylation. Our model uses this simplified form of Barkai and Leibler's model to reproduce the adaptation response in chemotaxis.

This model by Barkai and Leibler was used heavily in later years due to its robust properties [7]. More recently, Bray et al. [22], developed a deterministic model that solves ODEs that represent the signalling reactions in the pathway and predicts the bacterium motion in a two-dimensional (2D) environment.

The applicability of mathematical modelling depends on the number of particles in the system [30]. For example, in the Keller-Segel model the particles interacting with each other are unicellular organisms denoted by $(\mathrm{n})$ and the molecules of the stimuli denoted by $(\mathrm{S})$, both represented by a large number of particles [35]. If a system only contains a few particles, a better approach would be the agent-based approach, due to its ability to capture low-level details that would otherwise be overlooked in mathematical modelling.

\subsection{Agent-based Models}

Biological systems are traditionally modelled through mathematical modelling. However, in recent years a new technique is being adopted to describe biological systems. Agentbased modelling (ABM) is already used in numerous fields such as business (management, stock analysis, supply chains), terrorism (social determinants, prediction targets), military 
(command and control, attack and defence strategies) and Engineering and Planning (human movement, evacuation modelling) [43]. Now with advances in technology, we are able to use ABM in Biology as well. Some examples of ABM in Biology include: cancer research [1, immunology [24] developmental systems [42] emergent properties [30] and many more.

Agent-based modelling is also known as agent-based system (ABS) or individual-based modelling (IBM). The agent-based approach simulates the interactions of agents with other agents and their environment. These interactions often give rise to complex patterns, often referred to as the emergent phenomenon [18, 43]. This emergent property is not identifiable through individual agents, and it cannot be reduced to a system's parts as the phenomenon is dependent on the interactions between the agents [18, 43].

One of the most important aspects of ABM is the agent itself. The definition of an agent is a on going debate. Most modellers consider any independent component (software, object, model, etc.) with some sort of behaviour to be an agent [18, 43]. The behaviour can range from primitive reactive protocols to adaptive intelligence. The dispute revolves around the definition of the behaviour of an agent, such that in order for the independent component to be considered an agent, it must have adaptive intelligence [45]. Adaptive intelligence results in the agent having the ability to learn from the interaction with the environment and change its behaviour accordingly [50]. We can see that the definition of an agent revolves around the same basic concepts; it must be an independent component, and there must be some behaviour associated with the agent.

Casti [14] proposed that an agent should contain both base-level rules for behaviour and a higher-level protocol to "change the rules" (adaptive intelligence). The base-level rules provide a reaction to the environment while the higher-level protocol adapts to the environment. Essentially, the agents must be able to make independent decisions. This independent decision making is a fundamental feature of agents to ensure their behaviour is autonomous. 
It is generally accepted that an agent should also have the following properties [43]:

\section{An agent is identifiable}

Independent component of a system and self-contained. To be identifiable, the agent has a boundary and one can easily identify whether something is part or not part of an agent.

\section{An agent is situated}

Living in an environment where it has protocols on interaction with other agents and also protocols to respond to the environment.

\section{An agent is goal-directed}

It should have a goal that is associated with the behaviours.

\section{An agent is autonomous and self-directed}

The agent can function independently in the environment.

\section{An agent has behaviour rules}

The behaviour rules are a set of protocols that govern how the agent interacts with the environment and other agents.

An agent is not to be confused with particles in a system, otherwise known as a particle system. The difference is that an agent is diverse, heterogeneous, and dynamic in its behaviour in a system [30]. Agents differ by sophistication; sophistication of the agent is based on how much information is used by the agent before a decision is made. Adaptive or higher-level usually requires some form of memory, while base-level is a set of rules that govern the general behaviour and decision-making capabilities of an agent [30].

When one utilizes ABM, one must be mindful that $\mathrm{ABM}$ is a method that has high computation costs associated with its usage. The number of agents one can usually model in an ABM approach is the limiting factor. With each agent having its own set of properties 
such as interaction rules, physics, and graphics, the complexity and computation cost of the model increases exponentially as the number of agents in the model increase.

\subsection{Hybrid Models}

Modelling methods that utilize both mathematical modelling and ABM techniques are commonly referred to as hybrid modelling. There are two approaches to utilizing hybrid modelling. Both approaches involve a switching mechanism between the two methods. A model of the spread of an epidemic disease [17] can be seen using the first approach. This first approach initially considers infected components (individuals) as agents, but switches to the mathematical model when the number of infected in the model simulation rises above a threshold. The second approach involves the description of some system components as agents such as a bacterium cell, and other components as mathematical descriptions such as external chemicals (stimulus in chemotaxis) [30].

The use of hybrid models is becoming widespread, allowing for more complex systems to be modelled and simulated such as biological systems and their cellular processes. Hybrid modelling is already used in various areas such as tumor growth [51, and forest dynamics [37]. In terms of chemotaxis, some hybrid models already exist such as the model developed by Dallon and Othemer [25] which models chemotaxis of slime mold. The use of hybrid modelling allows for faster simulations than purely agent-based models which would treat every component as individual agents.

Hybrid modelling brings together the advantages of ABM and mathematical modelling. However, similar to purely agent-based models, it is often hard to obtain quantitative results, but with the correct balance between ABM and mathematical modelling in the hybrid model, it becomes easier to obtain quantitative results. 


\subsection{Multiscale Modelling}

Multiscale modelling involves modelling a system at different levels of detail. A basic example can be seen in Figure 0.2, and a complex example can be seen in the LINDSAY Virtual Human project [34]. Essentially, multiscaling allows for the ability to fly-through a simulation of a model and view the details of the model at different levels of magnification. The user would be able to interactively move into different layers of the model to reveal more details, such as the four layers in our model (Figure 0.1) or the "Cell Size and Scale" module presented by the Genetic Science Learning Centre (Figure 0.2 .

A relevant multiscale model was presented by Emonet et al. [26] with AgentCell. Their model, utilizes ABM and multiscale modelling. AgentCell accurately modelled chemotaxis in both a population level view and an individual bacterium level. AgentCell's population level and individual cell level are modelled as separate models that are simulated independent of each other. Our model incorporates the population model, individual cell model, and the molecular model into one model. In the next chapter, we present and describe our Multiscale E. coli chemotaxis model. 


\section{Chapter 4}

\section{The Escherichia coli Chemotaxis Model}

We have developed a generalized model of the chemotaxis pathway in E. coli. The model contains the key attributes and characteristics of the pathway which controls the locomotion of bacteria in a defined environment. This model was created using the hybrid-technique which combines mathematical and agent-based modelling methods.

The ability to switch between these two methods creates a smooth simulation. The mathematical model handles all the calculations related to the intracelluar and extracellular signals, and concentrations of molecules while the ABS handles the cell to cell interactions in low-level detail. This hybrid approach that our model utilizes ensures it is relatively computationally inexpensive as compared to a pure ABS approach. As previously mentioned in Chapter 3, ABM works well for small systems. However, we are modeling a biological pathway and the cellular processes, and to lower the computational resources required to run our model, the hybrid approach is needed.

Apart from modelling the cellular process in the chemotaxis pathway, we also have the multiscale aspect to consider. Each transition between our multiscale views is connected by a transition interface. Depending on which view is being transitioned to, there may be a switch between the ABS and mathematical model. This interface ensures that the next view is properly prepared before being rendered on the screen. This is important for our model as switching between different views during the simulation requires consistency.

\subsection{Architecture of the Model}

The architecture is separated into the following list of components. Figure 4.1 illustrates how the communication occurs between the components. 
Data Model: Contains the relevant data that is used in the simulation, and shared between the views of the simulation. All data pertaining to the chemotaxis model is synchronized in this module for central access.

Naked-eye View: In the first view, the simulation works based on a colour scheme. The colours are drawn onto the surface based on a grid system that covers the surface of the environment in the simulation.

Colony View: In the second view, the E. coli cells and stimulus is represented by a particle system. The particle system is used to generate the illusion of thousands or millions of cells and molecules in the environment.

Individual View: In the third view, the $E$. coli bacterium is an agent with its own distinct properties. The bacterium will follow a gradient similar to the naked-eye and colony view, but in this view we see the runs and tumbles of a single bacterium in detail.

Molecular View: The fourth view contains the molecular details of the chemotaxis cellular process. Each protein involved is an individual agent and they interact with other agents and the environment.

World: The world is the simulation environment. The world is generated by our LINDSAY Composer 2.0. This world is where all our cells, particles, and agents live.

Gradient: The gradient is a system used in our model to direct the E. coli movements. The first view makes it most obvious through the colours of red and green (repellent and attractant).

Flagella: The flagella rotation is based on the current trajectory of the bacterium. In the first and second view, the flagella is hidden as we are looking at the general chemotactic behaviour of the bacteria colony. In the third view, the flagella will rotate $\mathrm{CCW}$ if it is running straight or rotate $\mathrm{CW}$ if it is in the midst of a tumble. In the fourth view, we focus on the proteins affecting the flagella. 
Interface: The interface is the component responsible for the interactions between the user and the model.

Transition: The transition component is responsible for the processing and the transfer of information between the views within the model.

\subsubsection{Hybrid Modeling in Our Model}

In our model, the hybrid system works by changing the focus on the modeling system depending on the layer of detail we are currently in. In Figure 4.2, we can see how the different modeling systems are utilized within the different layers to contribute to the different behaviors and the resulting visualization from it. In the naked-eye and colony view, the mathematical modeling system is being utilized. Moreover, within these two views, we are observing a general behavior of chemotaxis. To generate the visuals, equations are used in calculations for the following components; stimulus concentrations, diffusion of stimulus, number of E. coli bacterium, and the rate of death of the E. coli bacterium itself, all of this information is fed into the graphics engine which then produces the visuals seen on the screen. For the next two layers, the individual and molecular view, the modeling system focus is changed to an agent-based approach as we are now observing the low-level details of chemotaxis.

Recall in Chapter 3.4, we mentioned two different approaches to hybrid modeling. Here in our model, we utilize a similar method to the first approach (Figure 4.2). When we are observing high-level details of chemotaxis, we use mathematical modeling. When we transition into the individual and molecular views, we switch to agent-based modeling as we are now observing low-level details. A transition between mathematical and agent-based modeling occurs during the transition between the colony and individual view (Figure 4.2). Although for the individual and molecular view the focus is on the low-level details of chemotaxis, mathematical modeling usage in these two views would contribute to a reduce of computation resources required to generate the visualizations. Ideally, the mathematical model will 
run in all four views to maximize the consistencies between all four views.

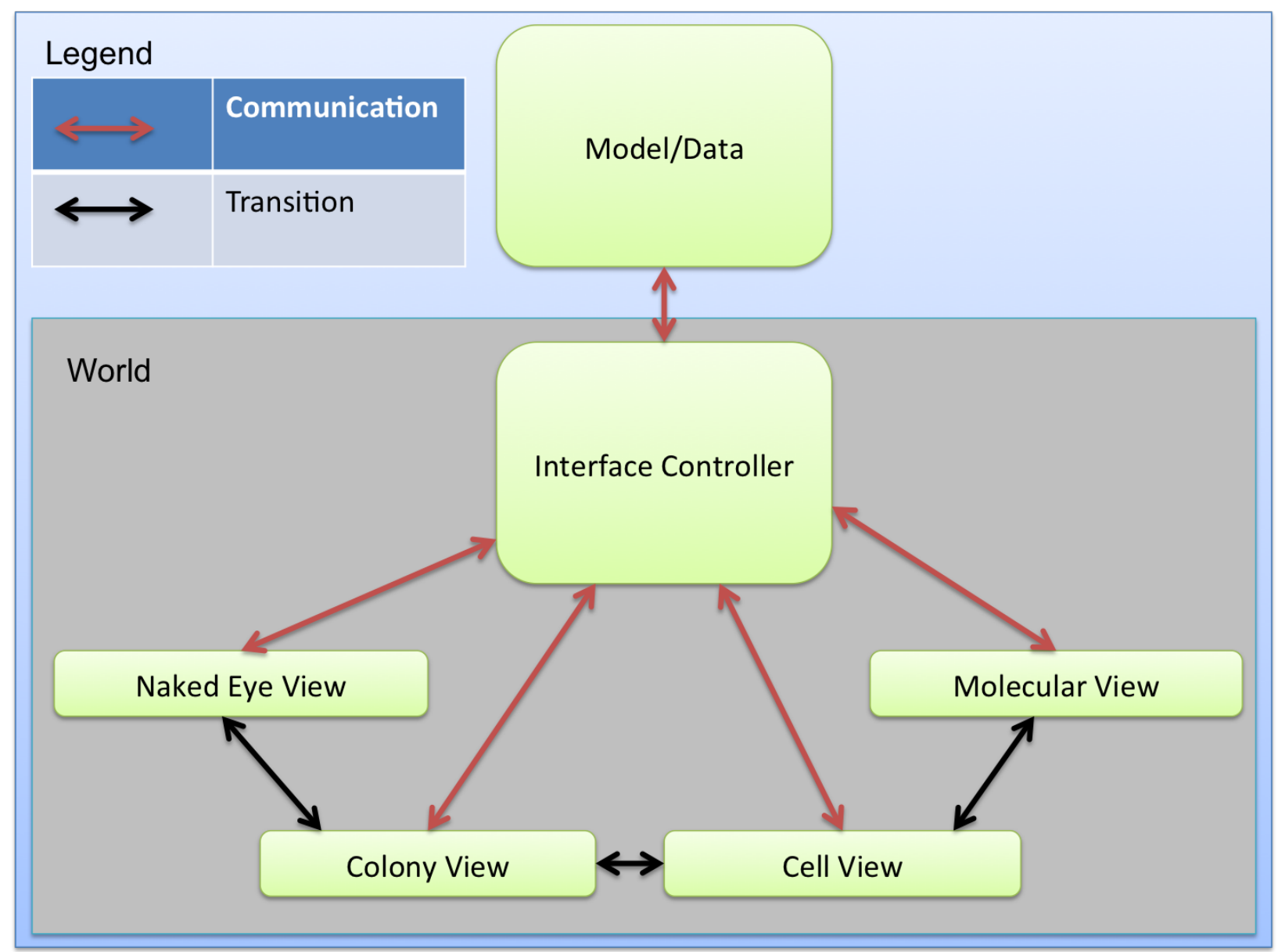

Figure 4.1: Communication hierarchy between the components in the model. The interface and views run in the world, which is the simulation environment. The data model is situated behind the scenes. Transition only occurs with the immediate views beside them, such that the naked-eye view cannot transition to the molecular view, and vice versa. All data between the views is communicated via the interface, there is no direct communication between the actual views.

\subsection{Layers of the Model and Functionality}

In our model, we have implemented four different layers, know as views. As seen in Figure 0.1, each layer of detail is a different magnification of the chemotaxis process (Chapter 1.4). Although each layer was built individually, and their functionality is independent of other layers, the data and information used by each layer is shared via a central data model. Any 


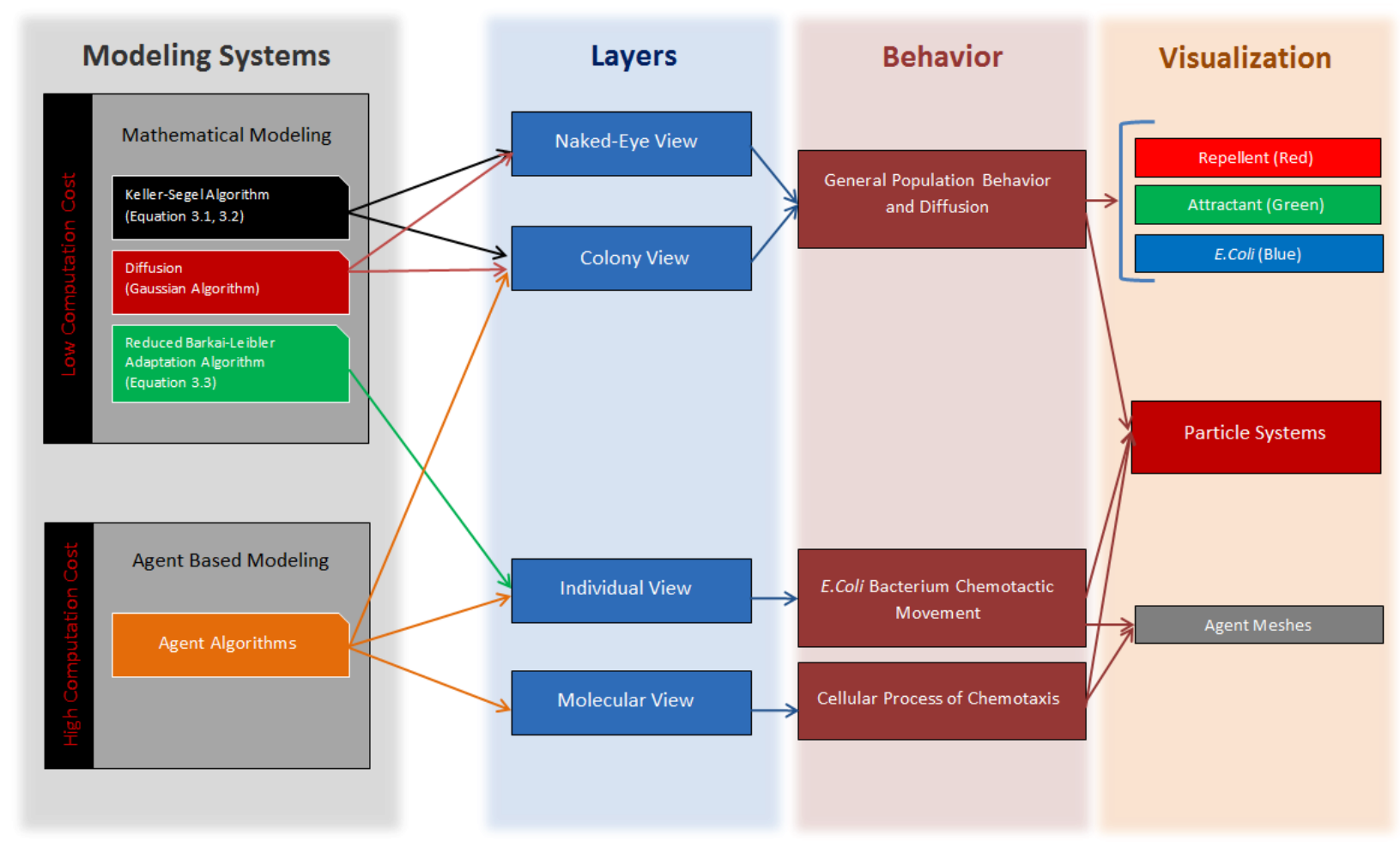

Figure 4.2: Brief overview on how the hybrid model system operates within our multiscale model. The first two layers, Naked-Eye View and Colony View, rely on mathematical modeling. The other two layers, Individual View and Molecular View, rely on agent-based modeling, along with an overlap between mathematical and agent-based in the Colony view and Individual view. 
modification such as the use or removal of data is communicated to this central data model, this is to ensure consistency during the transition between the different layers.

Each layer, starting from the top layer (naked-eye view) to the bottom layer (molecular view) progressively increases in complexity. In the following subsections we will look at each of the layers more in depth.

\subsubsection{Layer One and Layer Two}

The purpose of the first layer is to relay a visual representation of what an individual would see at the naked-eye level (naked-eye view). Figure 4.3A shows what we would see initially and Figure $4.3 \mathrm{~B}$ and Figure $4.3 \mathrm{C}$ shows what we expect to see after some time into the simulation. In a real lab environment, we would only see the E. coli colony, this is represented in our model by a blue colour to depict E. coli as can be seen in Figure 4.4A. However, to take this one step further, we can also see how the stimulus is diffusing in the environment.

The naked-eye view utilizes three colours: red, green, and blue. Blue represents the $E$.

coli colony(Figure 4.4A), green represents an attractant stimulus (Figure 4.4 B), and red represents a repellent stimulus (Figure 4.4C). As we can see with Figure 4.5, the E. coli can be seen moving in the environment in response to a stimulus. The stimulus will diffuse in a uniform manner, and over time the stimulus will saturate the environment.

As one would expect, with an attractant stimulus in the environment, the motion of the bacteria will be biased towards the location of the attractant as it contains the higher concentration of the attractant (Figure 4.5A). The same is true for a repellent stimulus, but rather than moving towards the stimulus, the E. coli colony will attempt to move away from the repellent (Figure $4.5 \mathrm{~B}$ ). Once the stimulus is fully diffused and saturated in the environment, the $E$. coli colony enters a random walk phase and waits for the next stimulus or eventual death.

The second layer (colony view) is similar to the first layer. The difference is rather than a coloured gradient displayed in the environment as we see in Figure $4.3 \mathrm{~B}$, the bacterium 
cells are displayed as shown by Figure $4.3 \mathrm{C}$. Each bacterium is a particle in the system. The properties have been set to mimic a random walk for each bacterium.

Both the first and second layers utilize a similar algorithm to simulate the motion of chemotaxis at the population level. The algorithm (Algorithm 1) in both views work via a diffusion algorithm. The simulation environment utilizes a grid based system. Initially, the user selects a location in the simulation environment. This selection is then translated into a $\mathrm{x}$ and $\mathrm{y}$ coordinate represented on a grid. The initial location is set to 100, a value that represents a percentage of the concentration at that particular grid cell, whether this be for the stimulus or the E. coli colony inoculation point. Both the stimulus and bacteria colony use the same diffusion algorithm except with different diffusion rates.

The diffusion algorithm (convolution algorithm) works by constantly diffusing each grid cell that contains a value of either the stimulus or E. coli into its adjacent cells (3x3 Gaussian kernel). In the naked-eye view (first layer), the value in each of the cells is converted to a percentage of the alpha value for each of the colours (red, green, blue). Whereas the initial value is 100 and the fully saturated diffused value is 0 , which corresponds to the alpha values of 1 to 0 for each of the colours as seen in Figure 4.4.

In the colony view (second layer), this value corresponds to how many particles should be generated for that particular cell in the grid. Essentially, each grid cell contains 3 values: an attractant, repellent, and E. coli value. The algorithm extracts these value's from each grid cell and converts it to either an alpha value for colour (naked-eye view) or the number of particles to be generated (colony view).

To mimic tumbling in chemotaxis, $E$. coli diffusion works by selecting a preferred direction (toward or away) from the current environment stimulus. From here, it will select a random grid cell that is in the area of the preferred direction as the new origin point for the bacterium. The difference between the stimulus diffusion and the E. coli diffusion is that in order to mimic the movement of $E$. coli, each new origin point of the bacteria has a set value of 


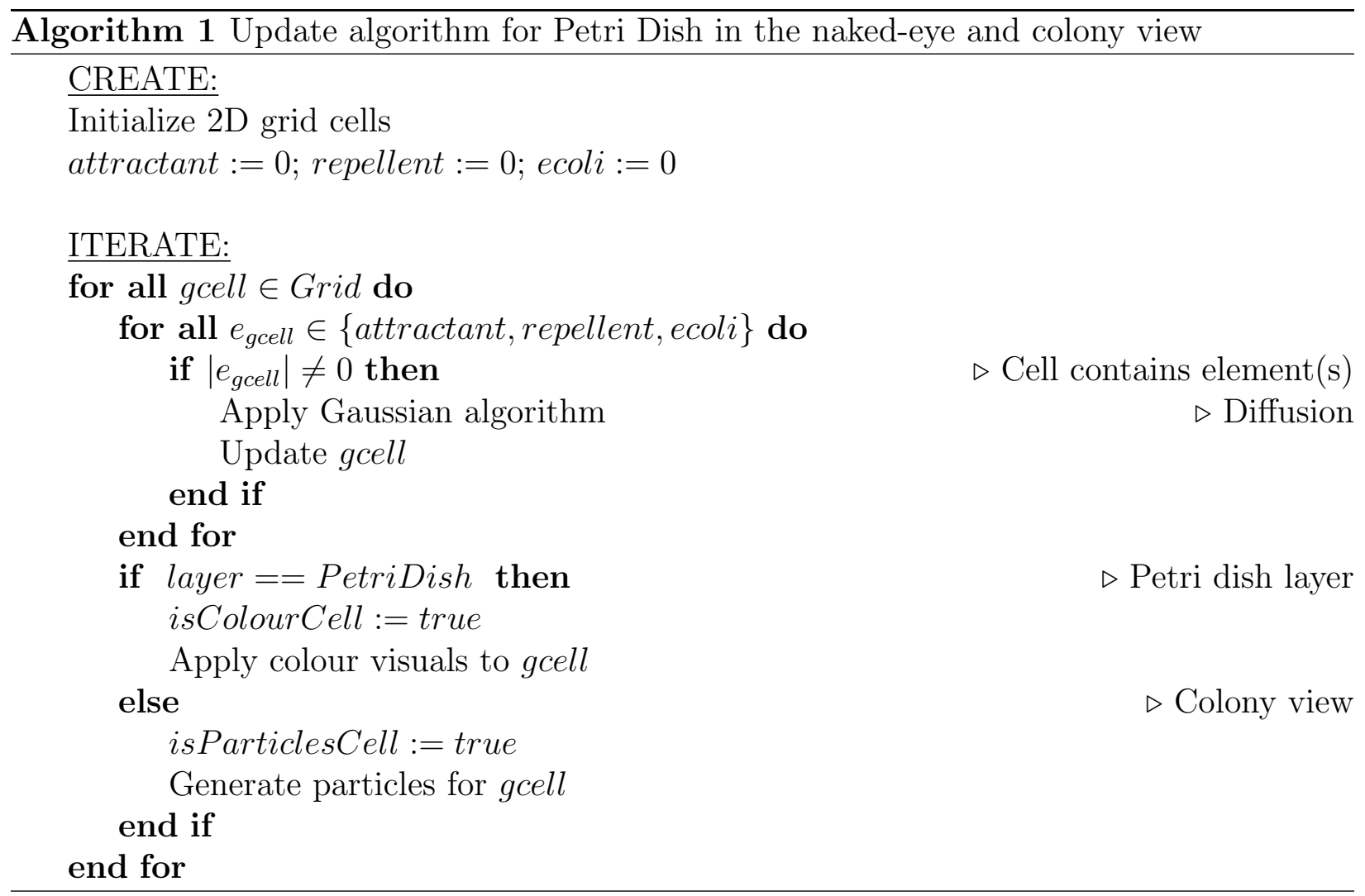

100 and then the diffusion algorithm is applied. The algorithm used in the naked-eye and colony view (Algorithm 1) removes the low-level details involved in the chemotaxis pathway. In the naked-eye and colony view, the interest is the population's general movement; hence the low-level details are abstracted into a general form to mimic the behaviour.

\subsubsection{Third Layer}

In the third layer (individual view), the algorithm is more complex than for the previous views. The algorithm implemented is a modified two-state chemotaxis model (Algorithm 2). Here, we have combined a two-state methylation model [7] with the two-state signal transduction cascade model [20]. The methylation model is used to mimic exact adaptation, while the signal transduction model is the driving algorithm for the run or tumble of the $E$. coli bacterium.

In this view, we modeled exact adaptation utilizing a two-state methylation model. How- 

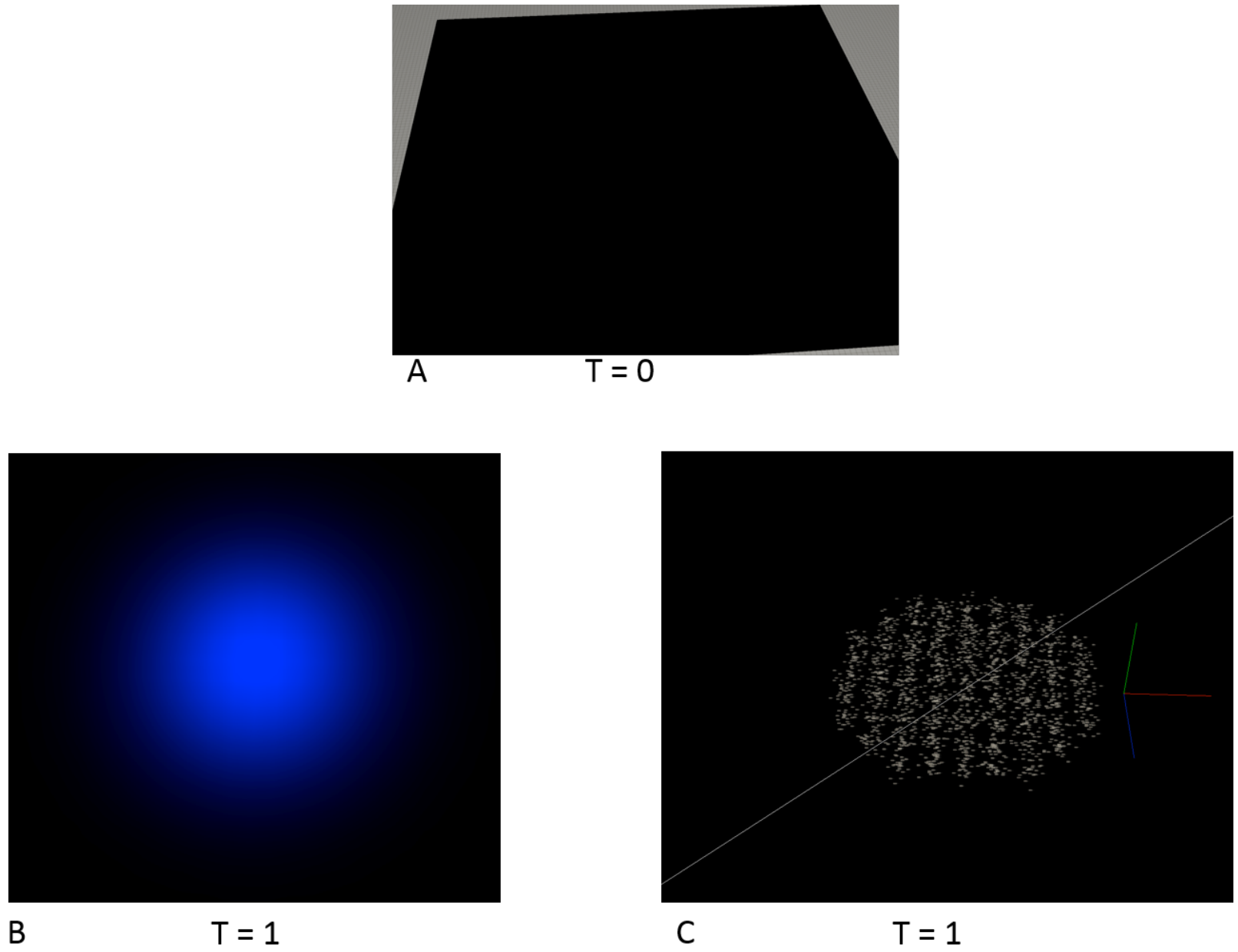

C

$\mathrm{T}=1$

Figure 4.3: A) What the simulation environment looks like at the start of the simulation (T $=0$ ). B) and C) What the simulation looks like at a time step after the simulation has been executed $(\mathrm{T}=1) \mathrm{B}$ ) shows the first layer (naked-eye) $\mathrm{C}$ ) shows the second layer (colony). 


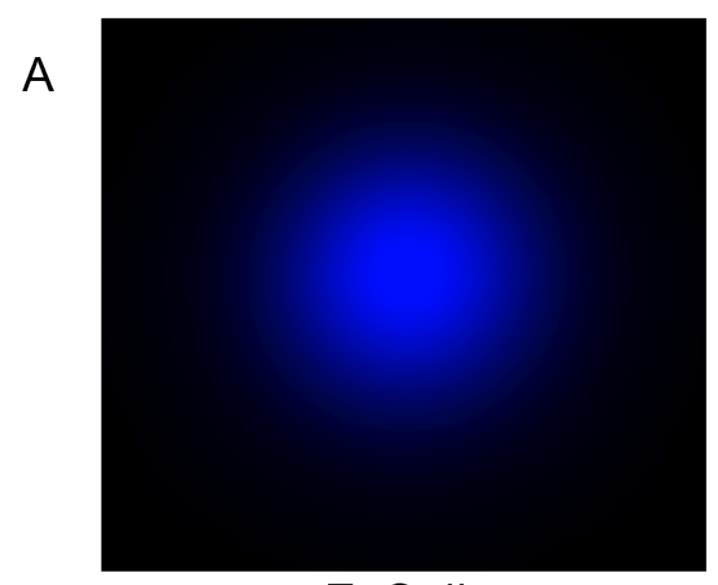

E. Coli

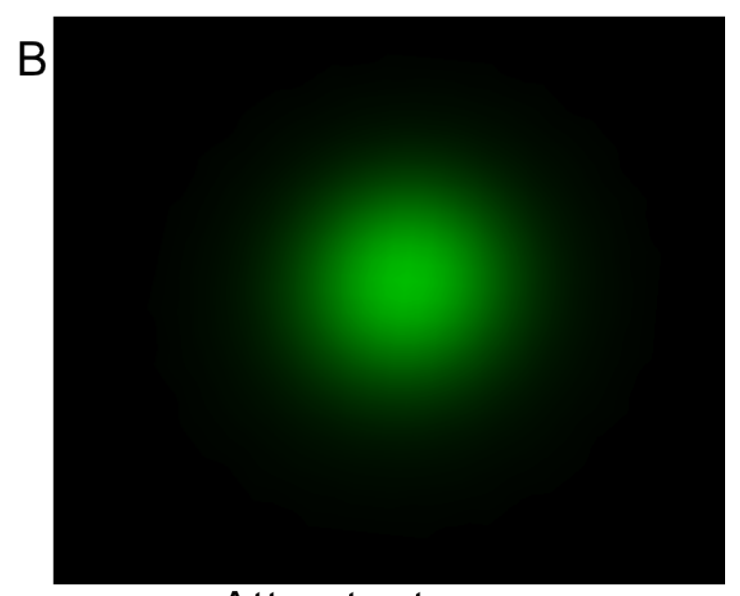

Attractant

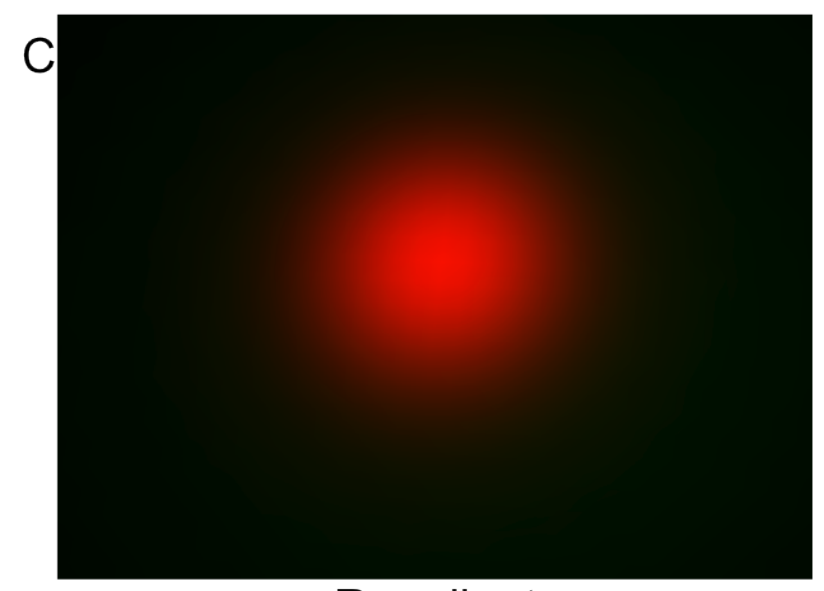

Repellent

Figure 4.4: A) E. coli gradient in the naked-eye view. B) Attractant gradient in the nakedeye view. C) Repellent gradient in the naked-eye view. 


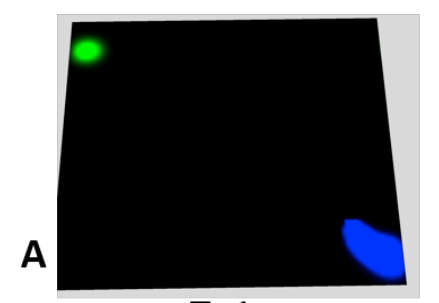

$\mathrm{T}=1$

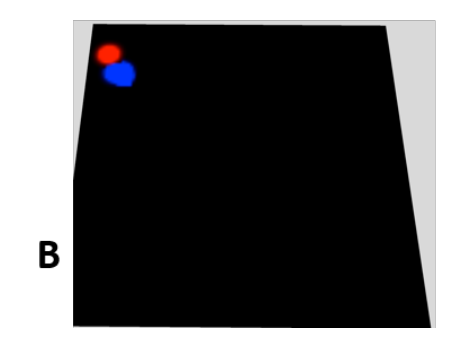

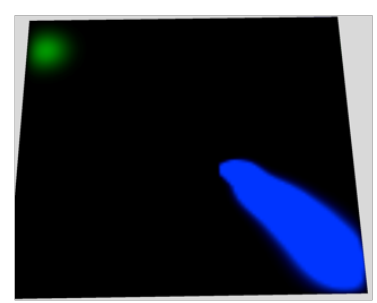

$\mathrm{T}=2$

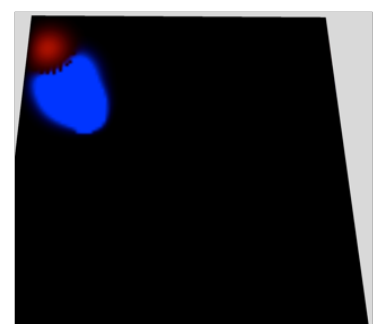

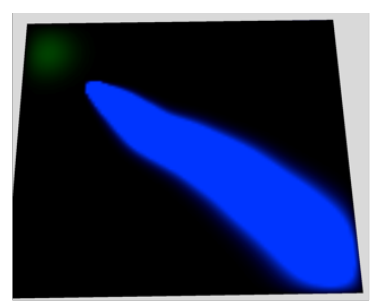

$\mathrm{T}=3$

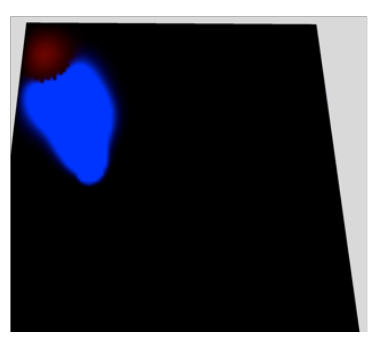

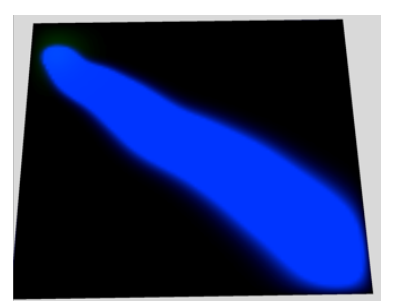

$\mathrm{T}=4$

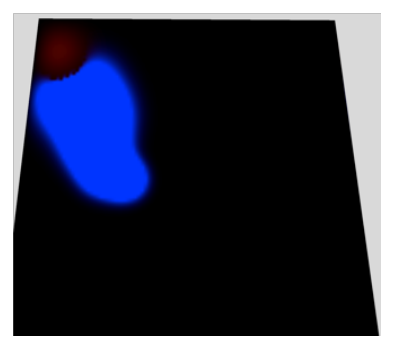

Figure 4.5: A) E. coli chemotactic movement towards an attractant stimulus in the nakedeye view over several time steps (T). B) E. coli chemotactic movement away from repellent stimulus in environment in the naked-eye view over several time steps $(\mathrm{T})$.

ever, the model is modified to incorporate the general signal transduction response in the chemotaxis pathway. The methylation response takes precedence over the signal transduction by directly affecting the activity state of the receptor (Algorithm 2). The state of the activity determines whether the receptor is avaliable for binding or not. The active state is when the molecules can bind, while in the inactive state the molecules cannot bind. The state of the receptor directly influences the rotation of the flagella.

Signal transduction works by taking experimental values [53] of CheY phosphorylation and dephosphorylation rates to determine the concentration of CheY-P vs. CheY in the bacterium at any given moment during the simulation. Rotation of the flagella is based on which concentration of proteins is higher within the bacterium. CheY-P increases the CW rotation which results in a tumble, while CheY increases CCW rotation which results in a run. As CheY-P concentration increases, the CheY concentration will decrease and when CheY concentration increases, CheY-P concentration decreases. Phosphorylation only occurs when CheAW complex is active. Dephosphorylation is constantly happening in the bacterium but at a slower rate compared to phosphorylation.

Similar to CheY, CheB has a phosphorylation rate that is directly affected by the receptor 


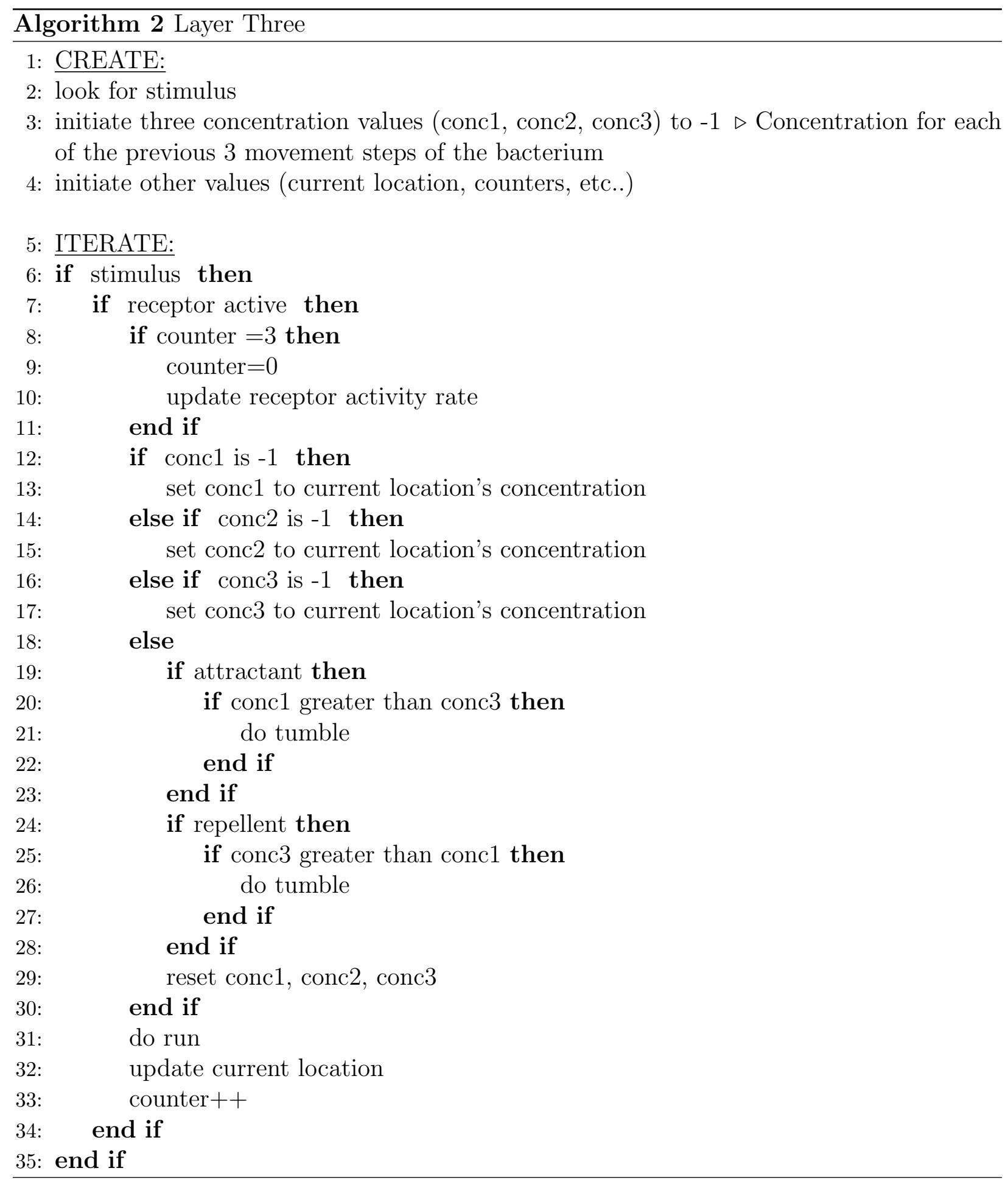


activity. Phosphorylation does not occur when CheAW is inactive (receptor inactive), this leads to a decrease in CheB-P concentration, which results in a faster methylation process than demethylation. This results in the reactivation of the receptor, and thereby reactivating CheAW.

\subsubsection{Fourth Layer}

The final layer in our model is the molecular view. In this layer, a representation of how the chemotaxis pathway works is presented in low-level detail. Both the excitation and adaptation responses are simulated and visualized here. The excitation response can be seen in Figure 4.6 and the adaptation response in Figure 4.7. Inside a real bacterium, we would see thousands upon thousands of protein interactions similar to Figure 4.8. Due to our limitations in computation power, modeling all these proteins and their interactions as individual agents would result in a very high computational cost. As the number of agents and interactions increase, the computation power also increases but at an exponential rate. The molecular view is used to show the finite details and interactions of the cellular process that is responsible for the chemotactic motion observed in bacteria.

In the next few subsections, we will describe our agent's roles and algorithms. See Figure 4.9 for a visual of our agents.

\subsubsection{CheY}

The CheY protein (Figure 4.9A) is an agent that can be in the following states:

- Random Walk: CheY is randomly moving within the bacterium between two points: the receptor and the motor.

- Phosphorylated: upon collision with an activated CheAW Complex, CheY will be phosphorylated, and its new end point of travel will be the motor. 


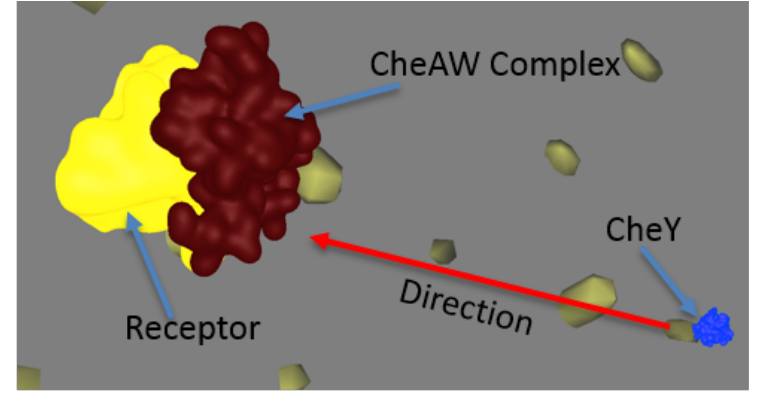

A

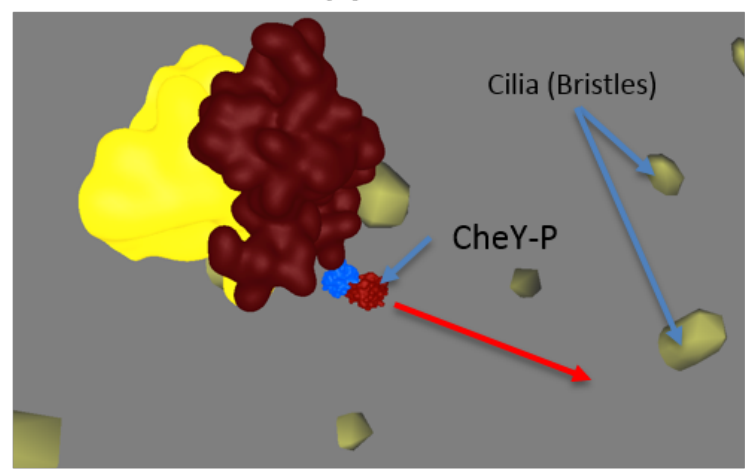

B

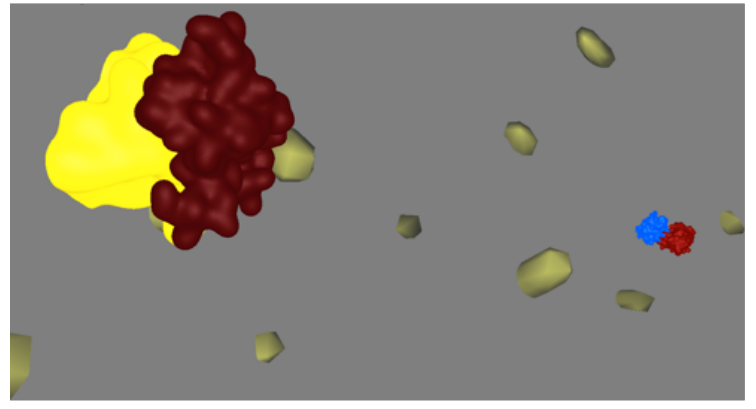

C
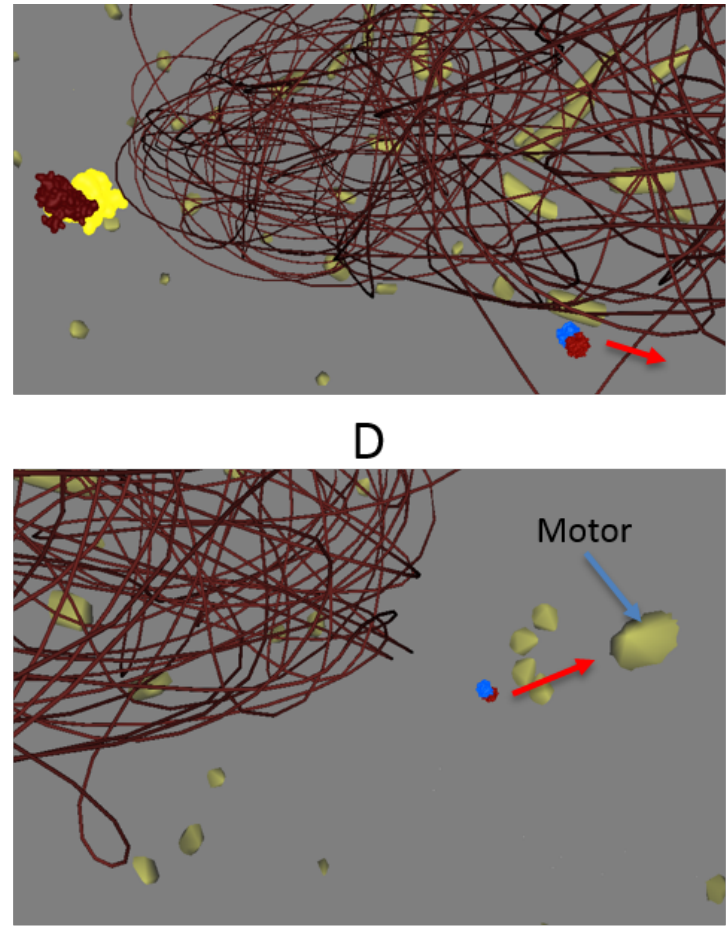

$E$

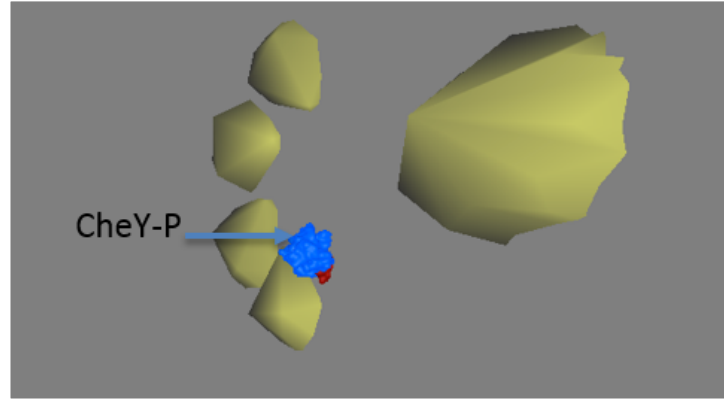

$\mathrm{F}$

Figure 4.6: The agent-Based representation of the excitation response. A) The CheY agent is active and moving towards the CheAW Complex. B) Upon collision with the CheAW Complex, CheY becomes phosphorylated and changes conformation to CheY-P. C) and D), the CheY-P agent moves away from the complex and towards the motor at the end of the bacterium. Finally, E) and F), CheY-P collides with the motor, upon collision CheY-P is bounded to the motor until it is dephosphorylated by CheZ. 


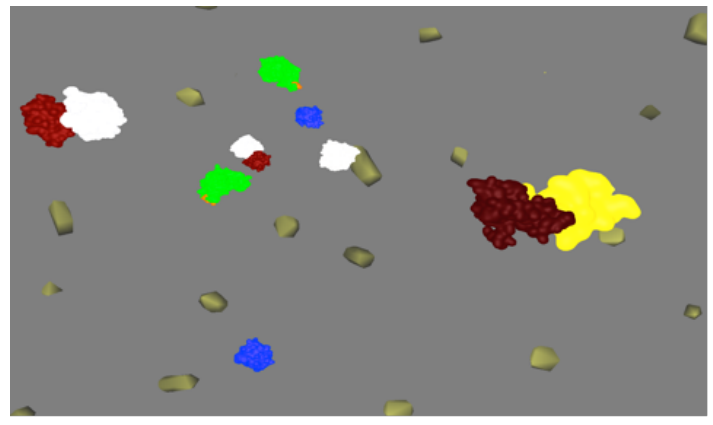

A

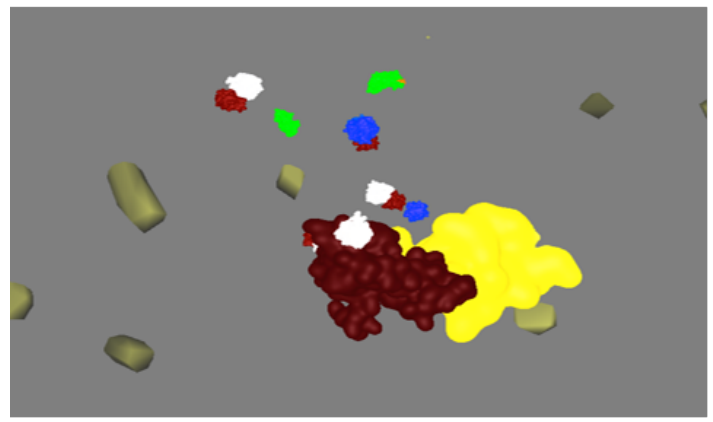

B

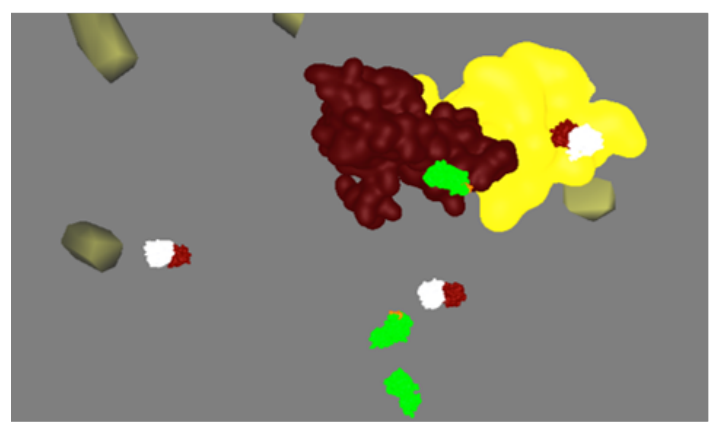

C

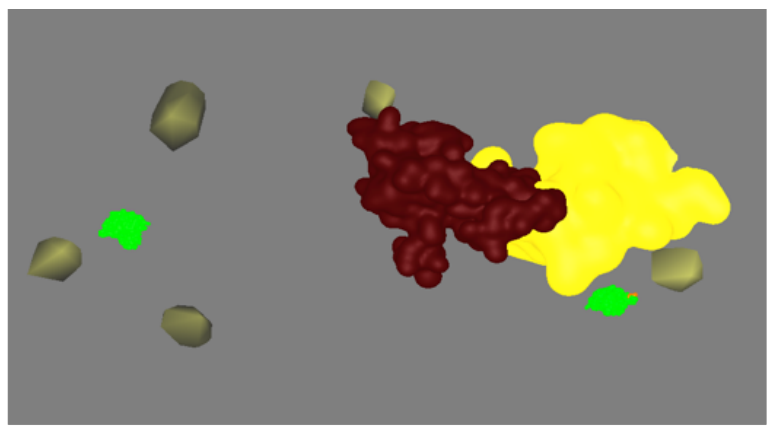

D

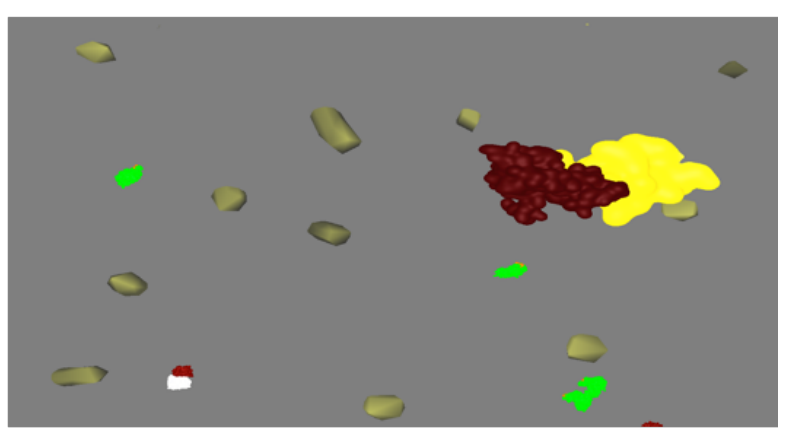

$E$

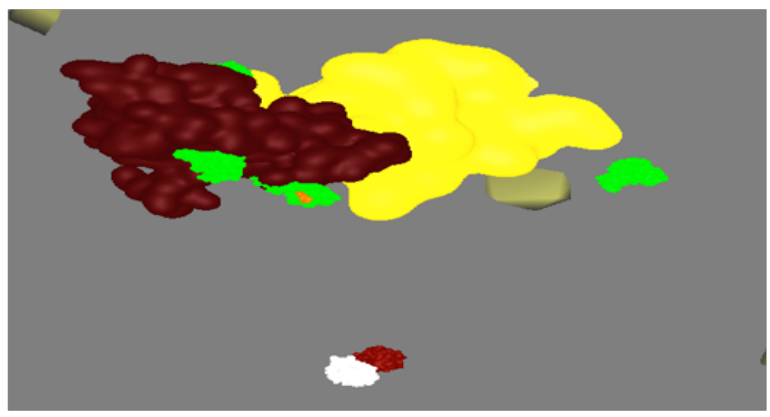

$\mathrm{F}$

Figure 4.7: The agent-Based representation of the Adaption response. CheR, CheR-M, CheB, CheB-P surrounds the vicinity of the CheAW Complex and Receptor. When CheR-M collides with the receptor, the $\mathrm{M}$ attaches to the receptor and CheR-M changes conformation to CheR. It will then wander away and after a period of time it becomes CheR-M again and moves towards the receptor to attempt to methylate it again. CheB moves towards the CheAW Complex to become phosphorylated. Once phosphorylated, CheB changes conformation to CheB-P and wanders for a period of time, and then it approaches the receptor to demethylate it. Behind the scenes, the simulation tracks the methyl groups currently attached to the receptor, and if the receptor has too many groups it will refuse the CheR-M from methylating it further. 


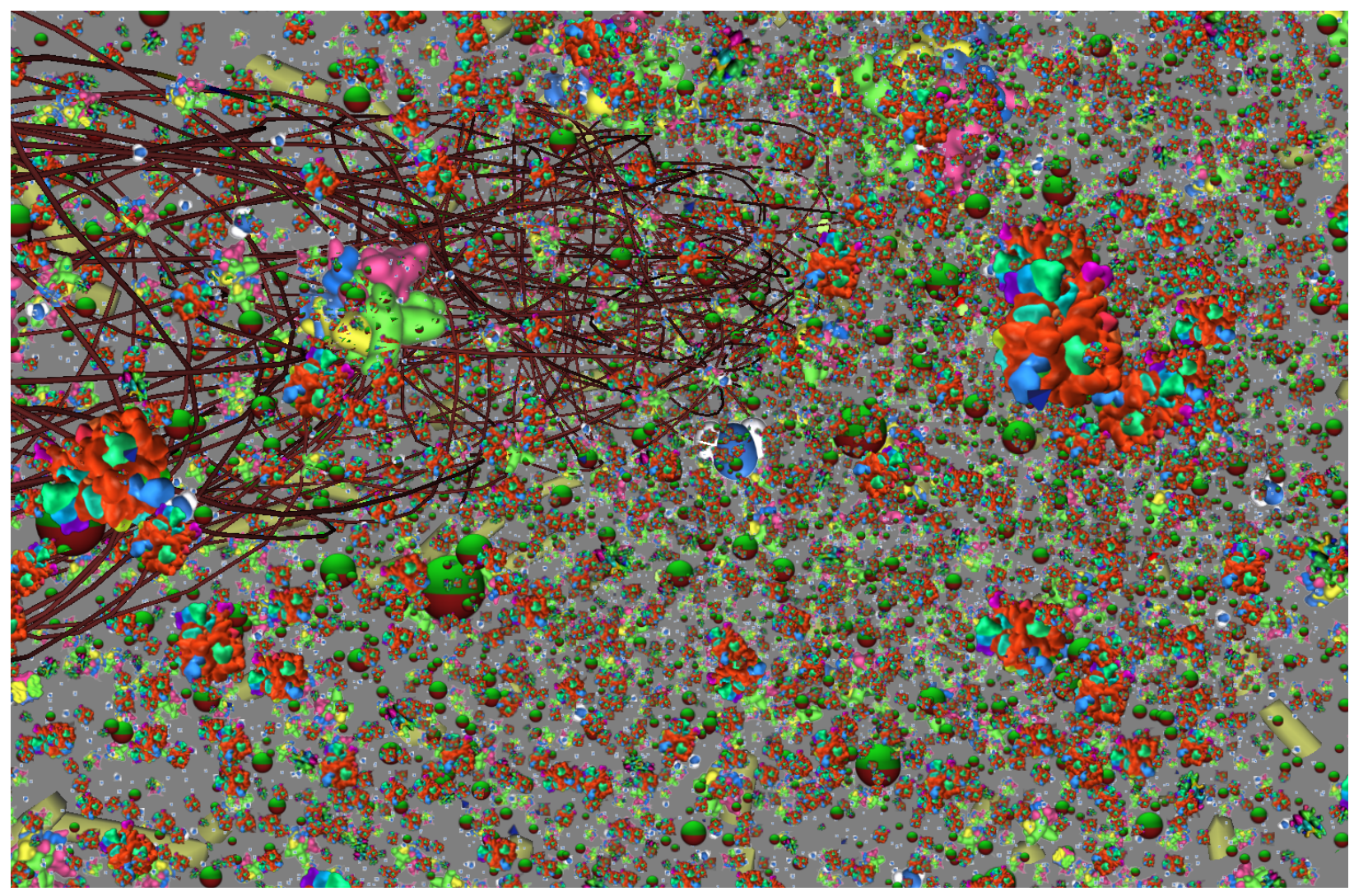

Figure 4.8: A glimpse of what it should look like inside a real bacterium. Several thousands of proteins. 


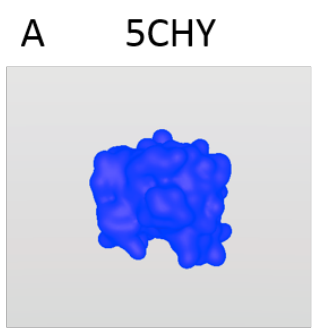

CheY

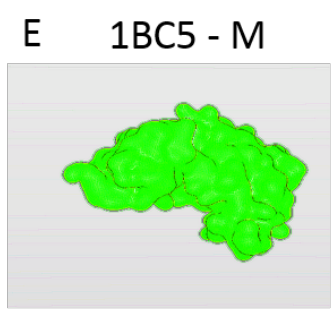

CheR

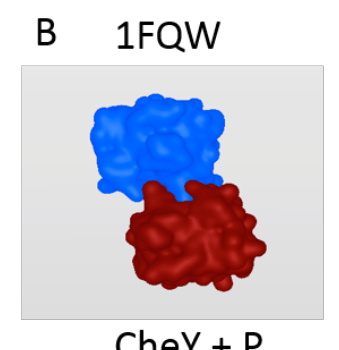

CheY + P

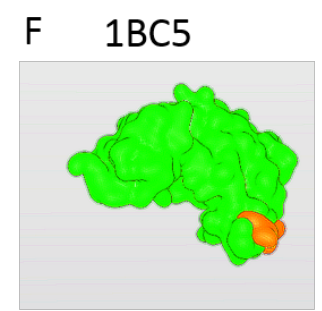

CheR-M

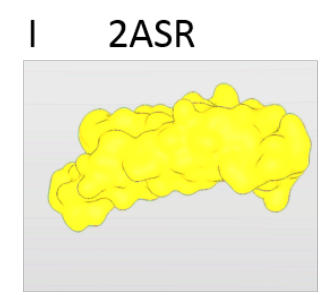

Receptor

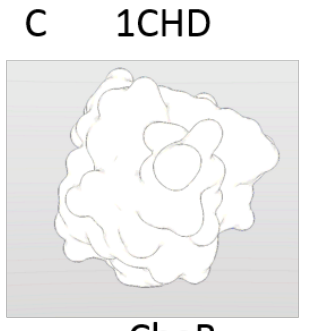

CheB

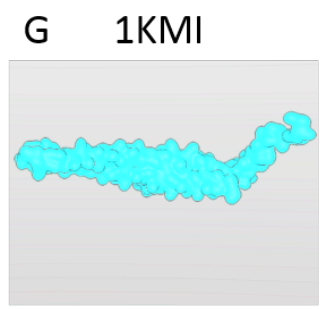

Chez

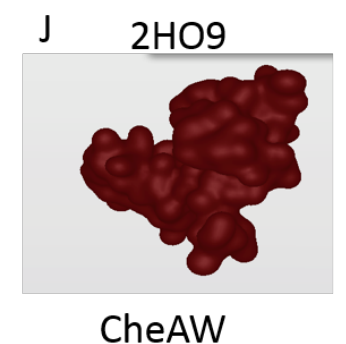

CheAW
D $1 \mathrm{CHD}+\mathrm{P}$

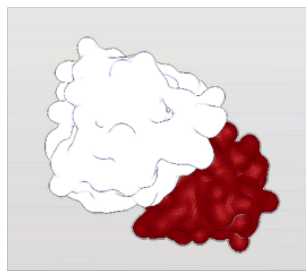

CheB $+\mathrm{P}$

$\mathrm{H} \quad 1 \mathrm{KMI}+\mathrm{P}$

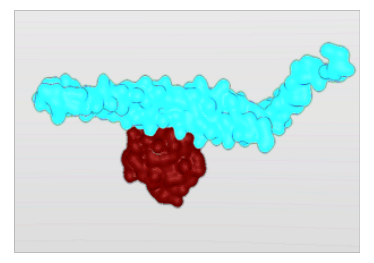

CheZ + P

Figure 4.9: A table of our chemotaxis Proteins as agents in the molecular layer of the simulation. The agents meshes were imported using the associated PDB ID from the PDB (http://www.rcsb.org/.) 
- Unphosphorylated: upon collision with a CheZ agent, if CheY is in the state of phosphorylated, it will be dephosphorylated and the new travel point of CheY will be set to the CheAW Complex.

- Bounded: if CheY is phosphorylated, upon collision with the motor complex, it will bind to the complex until it becomes dephosphorylated.

The conformation change between phosphorylated CheY and unphosphorylated CheY is represented by two different 3D structures obtained from the RCSB Protein Data Bank (PDB http://www.rcsb.org/) (5CHY and 1FQW) seen in Figure 4.9AB. The pseudo-code for the CheY agent is presented in algorithm 3 .

\subsubsection{CheB}

CheB similar to CheY is phosphorylated by CheAW complex. CheB (Figure $4.9 \mathrm{C}$ ) can be found in the following states:

- Random Walk: CheB is wandering in the cytoplasm of the bacterium.

- Phosphorylated: Colliding with the CheAW Complex will cause phosphorylation of CheB and the new end point to be the Receptor.

- Unphosphorylated: CheB-P colliding with the receptor will present a chance to demethylate the receptor. In this case, CheB-P will lose the Pgroup, and become unphosphorylated.

- Collision Not Allowed: This state occurs after CheB changes state between phosphorylated and unphosphorylated, this state allows for a delay in collision as the CheAW complex is attached to the receptor.

CheB phosphorylated and unphosphorylated states corresponds to a conformation change of the agent (Figure $4.9 \mathrm{C}, \mathrm{D})$. Temporary disabling collision ability is required because the 


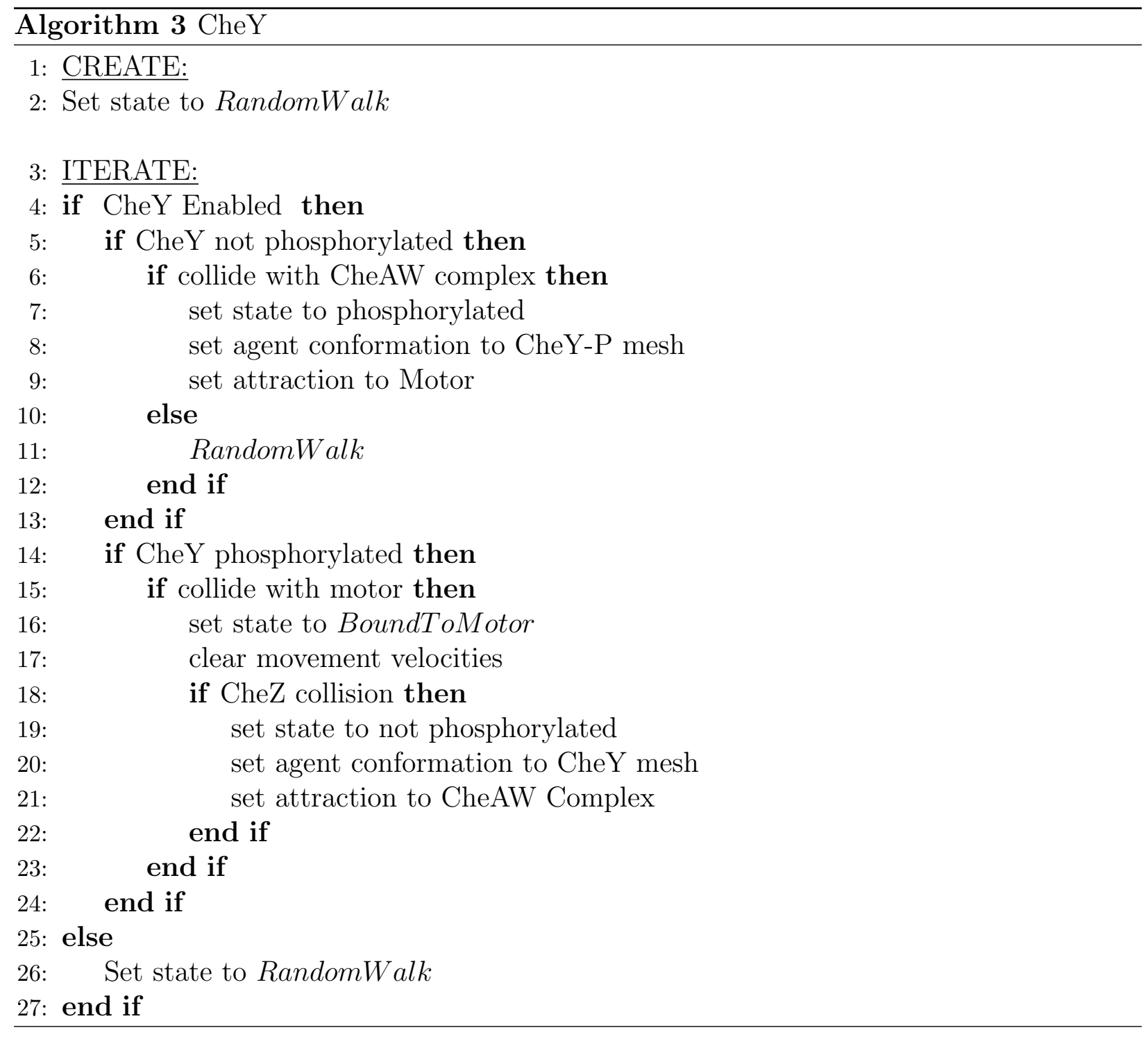


physics engine registers an object collision every 0.3 seconds [8]. To allow for sufficient time delay between the collisions of CheB and the CheAW complex and Receptor, this was a required state of CheB. In this state, CheB is wandering away from both of these target points. As algorithm 4 shows for the CheB agent, once the timer count-down reaches 0 , CheB exits this state. Based on whether it is phosphorylated or unphosphorylated, it will travel towards the intended agent.

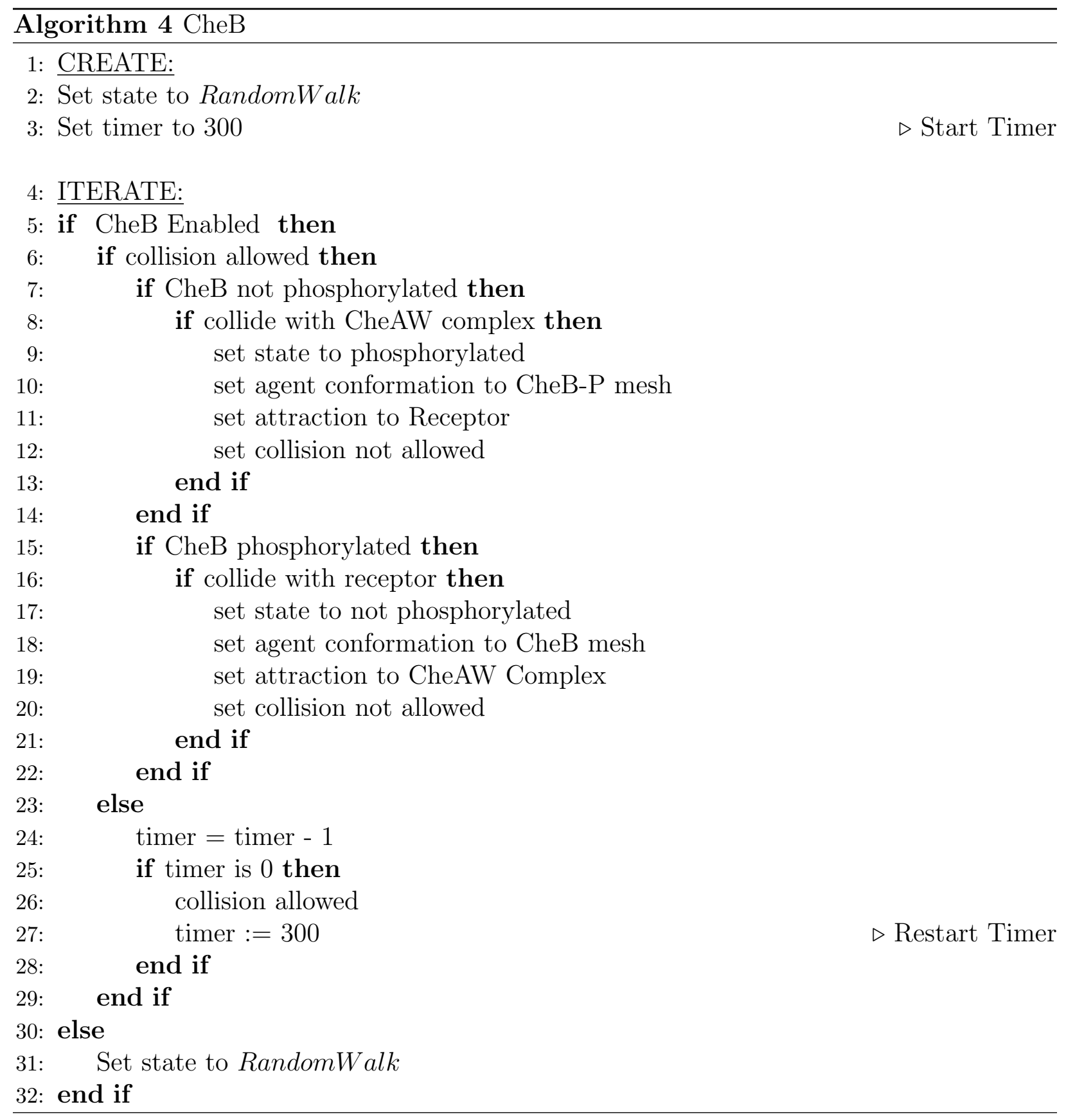




\subsubsection{CheZ and CheR}

CheZ and CheR (Figure $4.9 \mathrm{E}, \mathrm{G}$ ) are agents with not many states. CheZ is responsible for dephosphorylating CheY-P and CheR is responsible for adding methyl groups to the receptor. Both CheZ and CheR are similar such that they have one specific task. Both agents wander around the bacterium looking to complete its designated task.

CheZ is set to be attracted to the motor complex, CheZ wanders around the motor looking for collisions with phosphorylated CheY. Upon collision with a CheY-P agent, it will remove the $\mathrm{P}$ group from CheY and enter a period of inactivity. Algorithm 5 shows the pseudo-code implementation of CheZ.

CheR is set to be attracted to the receptor. During its wandering period, if it collides with the receptor, it checks whether or not the receptor has the maximum number of methyl groups already. If not, it will add a methyl group to the receptor and enter a period of inactivity, similar to after CheZ dephosphorylates CheY-P. CheR agents pseudo-code can be seen in Algorithm 6.

\subsubsection{CheAW Complex and Receptor}

The CheAW complex is a static agent. Its sole purpose (when activated) is to phosphorylate CheY and CheB. The CheAW activity state is directly affected by the receptors activity state.

\subsubsection{Motor}

The motor complex controls the flagella. If the number of CheY-P molecules bound to the motor is greater than $50 \%$ of the binding sites on the motor complex, it will cause the flagella to rotate $\mathrm{CW}$. The number of binding sites on the complex in our model is considered to be the number of CheY and CheY-P agents in the simulation space. 


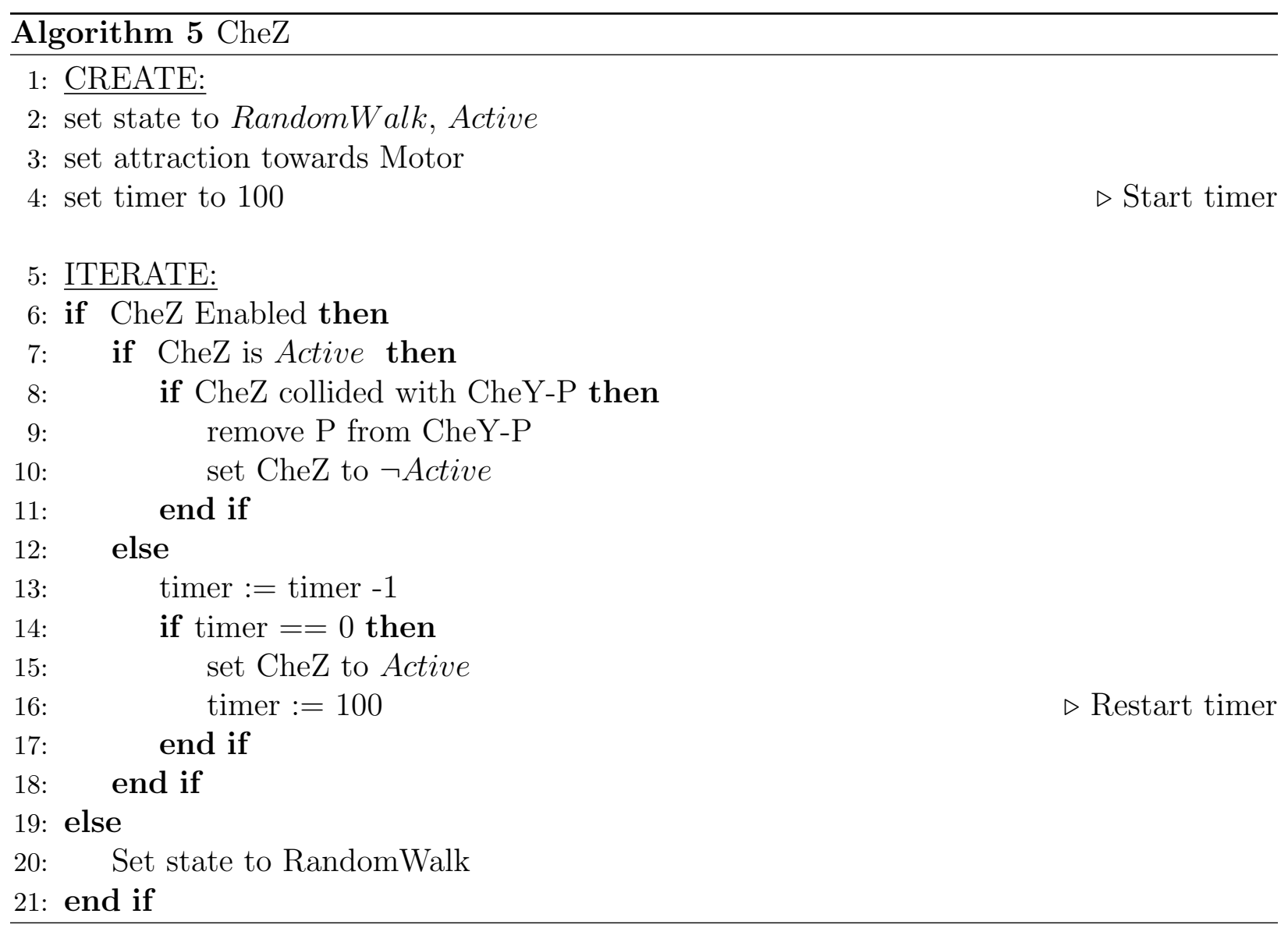

\subsection{Challenges}

Throughout the development of this project, several challenges were encountered. These challenges can be categorized into one of two parts: a biological understanding or a technical obstacle. As the project progressed, these challenges often merged and generated a new set of challenges. In the following sections, specific challenges to each of the categories are further explored.

\subsubsection{Biological Challenges}

The main challenge here was the organization of the data available. Chemotaxis is a wellstudied process with research dating back to 1880 [13]. The challenge was to combine all this data into an organized structure that could be implemented into the simulation.

In addition, understanding the exact cellular process of chemotaxis was difficult. Chemo- 


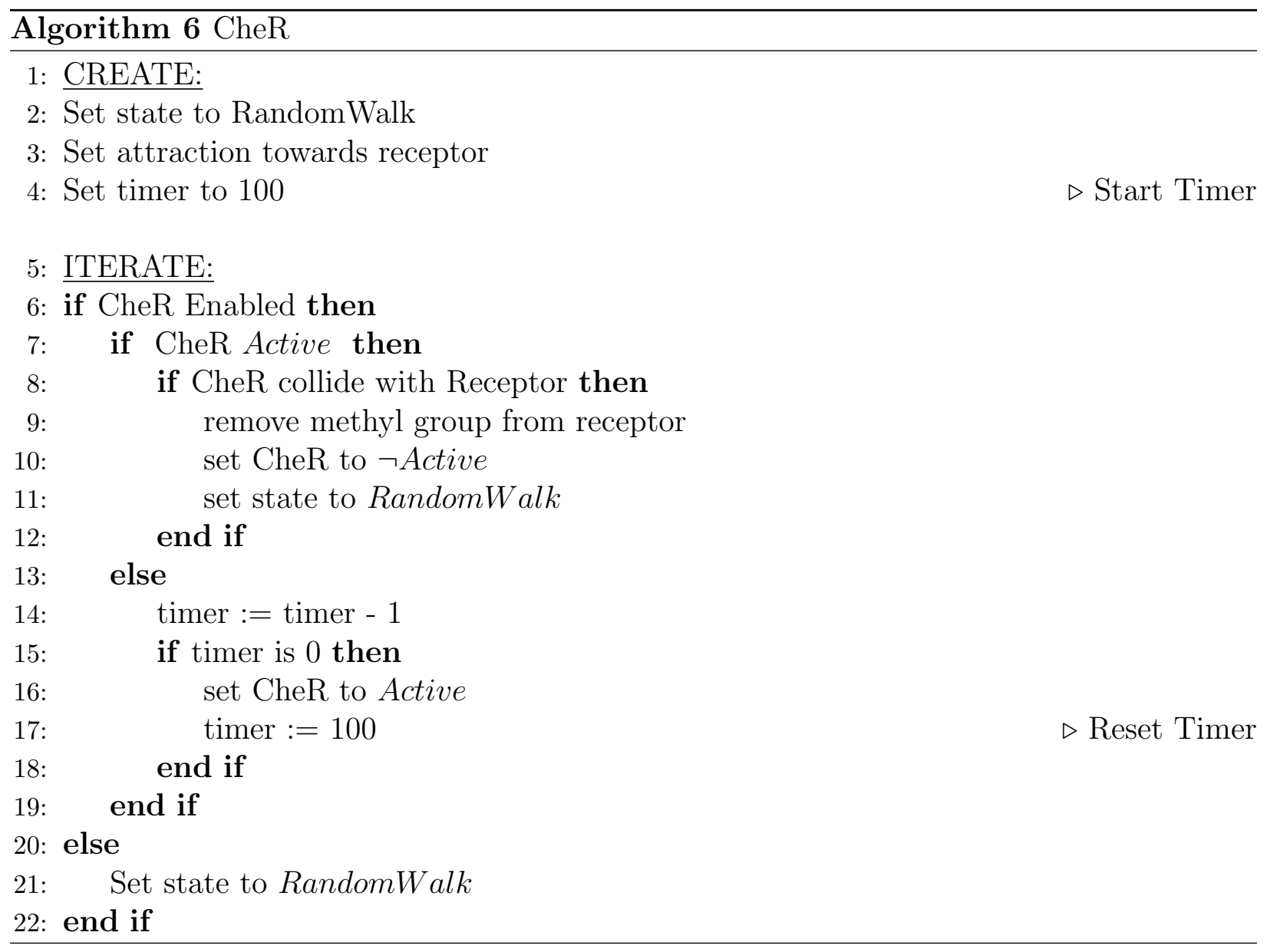


taxis is composed of two responses (discussed in Chapter 2.1): Excitation and Adaptation. Understanding how these responses function separately and together proved to be a difficult task. These two responses were often studied and modelled separately [26]. The task was to understand how these responses functioned and how they relate to each other in the chemotaxis pathway.

The challenges that followed involved understanding the mathematical model behind the two responses. Numerous papers [57] modelled the biochemical reactions into a mathematical model. These cellular processes are often given in a series of mathematical equations. Myself, not being particularly strong in the field of mathematics, had difficulty comprehending the mathematical equations and reasoning behind the model. Although these mathematical models are derived from the same theoretical models, they can differ greatly [13, 36].

Moreover, another challenge was trying to pinpoint specific information related to the problem. Due to an enormous amount of data and information on chemotaxis, it was difficult to find information specific to the problem being investigated.

\subsubsection{Technical Challenges}

Technical challenges involved everything from computer problems to the actual implementation of the model. The model used much more resources than expected. As a result, this led to multiple problems; however, the main problem was a slow simulation due to the high computation resource cost associated with the ABM.

Several models exist for implementing chemotaxis [26] (as discussed in Chapter 3), the challenge was understanding their advantages and disadvantages. Many of the models implemented only one of the responses as explained in Chapter 3 [26]. The goal for our model was to be as comprehensive as possible with the pathway, which requires the inclusion of both responses as chemotaxis does not function properly solely based on one of the responses. Each response has its own specific function within the pathway, here the challenge was to connect these two separate responses into a single functional implementation. 


\subsubsection{Combined Challenges}

The biological and technical challenges combined into more complicated challenges. One of them being abstraction. The question now is how much of the Biology should be abstracted. Too much abstraction would result in a significant amount of biological information lost, and limited abstraction would lead to a computationally heavy simulation. The task faced was to find the balance between the two.

These challenges were difficult to overcome, but in the end all these challenges were solved resulting in the production of our 3D Multiscale Chemotaxis in E. coli Model. 


\section{Chapter 5}

\section{Interaction}

One of the distinguishing features of our model from previous chemotaxis models is our interactive component. Unlike other models, ours is interactive and in real-time. As the simulation runs, the user can interact with the simulation, and changes are reflected immediately. Most of the interaction has to do with disabling specific proteins in the chemotactic pathway. Each of the proteins have a specific task in the process and with the user ability to switch these proteins on and off, we can see in real-time the prediction of what may happen.

In our model, the user has the ability to switch off the receptor, CheAW, CheY, CheB, CheR and CheZ in any view of our multiscale model (Figure 6.6). Each of these is involved in our algorithm that generates the chemotactic response in the environment. Table 2.1 summarizes the effect that each of the proteins have on the model. In the following section, each protein role will be described and its effect if the protein is disabled. Our model only covers 5 chemotactic proteins, but other proteins involved can be readily added.

Another defining interaction of our model is the ability to travel through different scenes. In our case, each of these scenes represent different layers, otherwise known as views (Figure 0.1. Each of the views, progressively reveal more details of chemotaxis from high-level to low-level details.

\subsection{The Proteins}

CheY, is the main protein responsible for the chemotactic behaviour. Once CheY is phosphorylated, it will bind to the motor of the flagella in the cell. This binding will bias the rotation of the flagella clockwise. Recall back in Chapter 2.3.1, the clockwise rotation results in tumbling of the E. coli. Switching off CheY, will effectively eliminate the tumbling ability 
of the cell. Without CheY, there can be no CheY-P, and without any CheY-P to bind to the motor, the cell will run in the direction it is facing when it spawns in the environment.

The CheAW complex is bound to the receptor end that is inside the cell. CheAW is responsible for the phosphorylation of $\mathrm{CheY}$ and CheB. This phosphorylation process creates CheY-P and CheB-P, both very important in the chemotactic pathway. The CheY-P helps regulate the rotation of the flagella, while the CheB-P is part of the methylation response in the pathway. If the CheAW complex is disabled, no phosphorylation process occurs and no CheY-P or CheB-P is created. Essentially what should happen is a constant run of the bacterium ignoring conditions in its environment (Figure 6.12). The receptor, directly affects the CheAW complex, disabling the receptor would have the same affect as disabling the CheAW complex.

CheZ, is responsible for dephosphorylation of CheY-P. CheAW phosphorylates while CheZ dephosphorylates. This is vital as it keeps the equilibrium of the concentration of CheY and CheY-P within the cell. This equilibrium can shift depending on the stimulus in the environment; however without CheZ, the environment stimulus would not matter. Since, there is no process to dephosphorylate CheY-P, the equilibrium will eventually completely shift to a $100 \%$ concentration of CheY-P.

Another protein is CheB. As mentioned, the CheB protein is part of the methylation response in the chemotactic pathway. It is responsible for the demethylation of the receptor to deactivate it. In order to do this, CheB is phosphorylated by CheAW. In synergy with CheR, these two proteins create the negative feedback loop in the chemotactic pathway that is responsible for the exact adaptation phenomenon. Without CheB, there is no CheB-P, which leads to no demethylation of the receptor.

The other protein that the user has control over is CheR. CheB is responsible for demethylation while CheR is responsible for methylation. In our algorithm, the receptor has two states; active and non-active. CheR methylates the receptor to activate it. Without CheR, 
the receptor would be inactivated and stay inactivated. Since methylation cannot occur without CheR, the receptor stays inactivated and this leads to a $100 \%$ concentration of CheY, which will bias the rotation of the flagella to be CCW.

\subsection{The World}

Controlling of the 3D simulation world is a very important part of our model. This interactive component gives the user the ability to choose where the E. coli cells spawn from and where the stimulus should originate. The user selects from the tool bar of the LINDSAY simulation

environment (Figure 5.1) the component they want to insert into the environment. In a simple point and click method, the selection will be generated in the environment at the specific location the user has chosen.

There are 3 components that the user can add: The E. coli, the Attractant, and Repellent. Initially, nothing will be shown on the environment until the simulation begins. The ability to pick and choose where these components should start on the environment gives the user a greater degree of flexibility to observe the chemotactic response from different views and angles. If a mistake was made during the placement of the component, the user can always reselect and choose a new location. The new location for the component will overwrite the previous one in the simulation. The addition of all 3 components at once is also a possibility, the key here is to give the user the flexibility to interact with the simulation.

\subsection{Adding and Removing Agents}

In addition to enabling and disabling agents in the simulation, the user has the ability to also add and remove agents to and from the simulation (Figure 5.2). As new agents are added, they become part of the simulation immediately. Adding and removing agents will increase and decrease the number of collisions between the agents which will directly affect how the simulation plays out. For example, adding more CheZ agents will effectively cause 


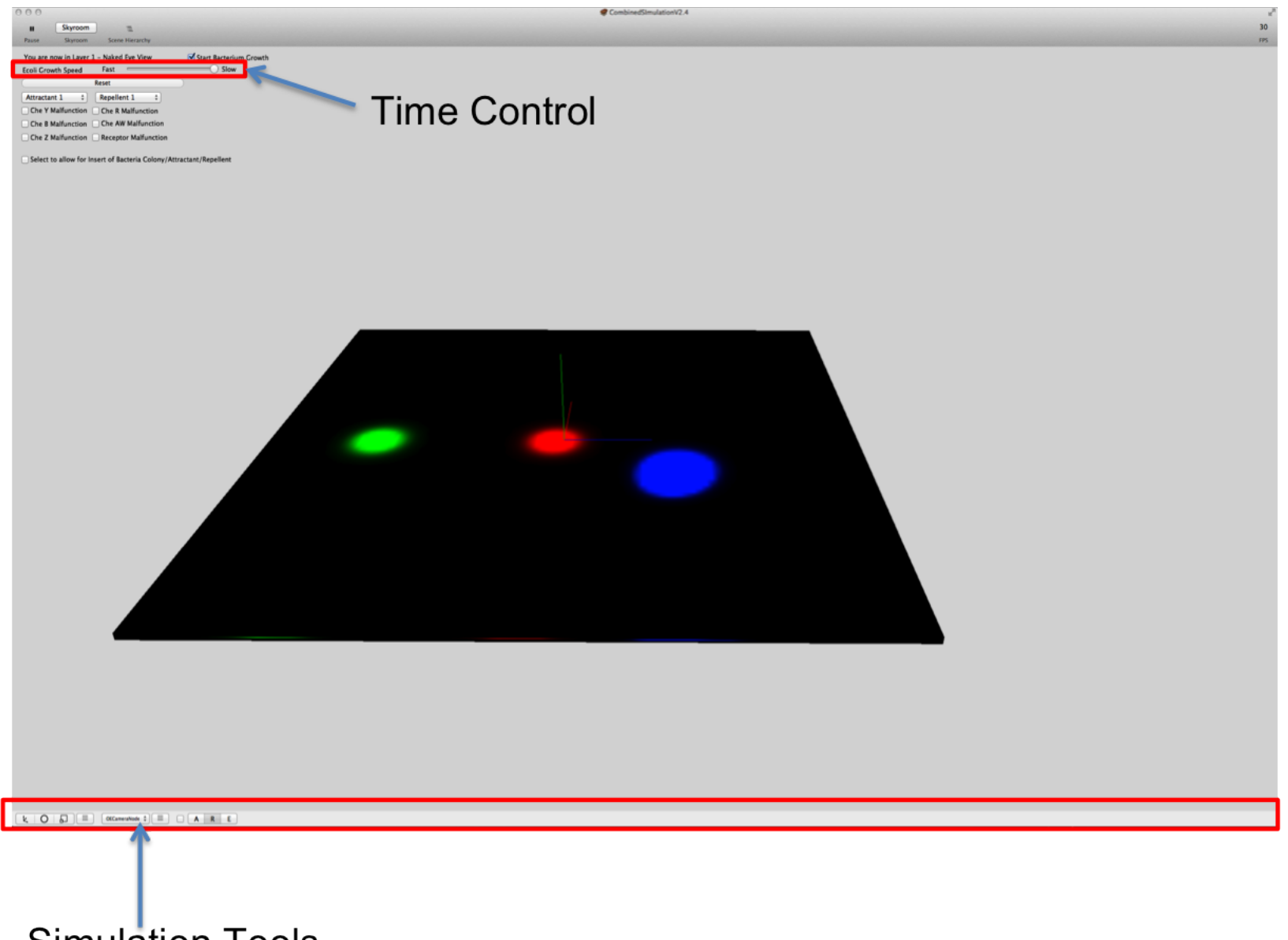

\section{Simulation Tools}

Figure 5.1: The Simulation Toolbar in Lindsay Composer used to control elements (Attractant, Repellent, E. coli ) in the naked-eye and colony view of the Model, and the Time Control bar to control the speed of simulation. 
the flagella to bias the CCW rotation more due to the lower concentration of CheY-P agents that could possibly bind to the motor complex.

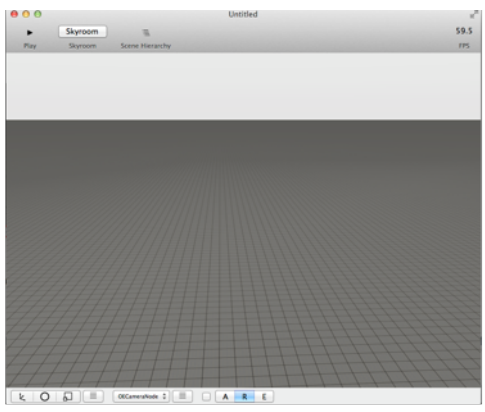

A

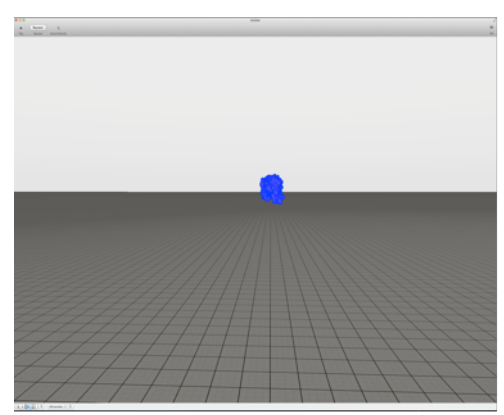

D

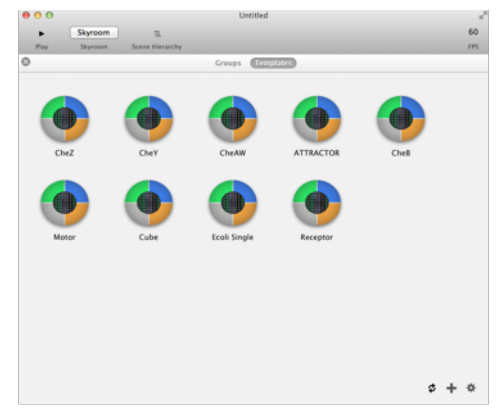

B

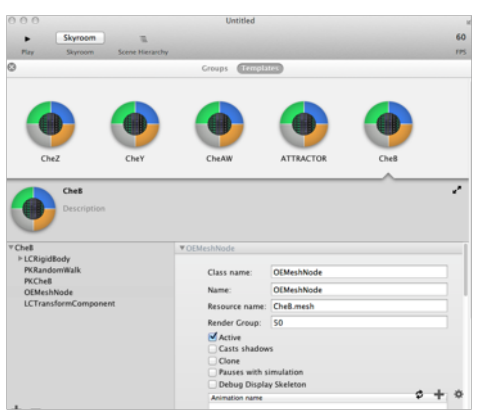

$E$

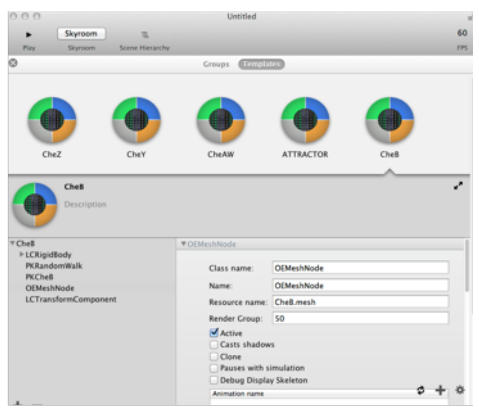

C

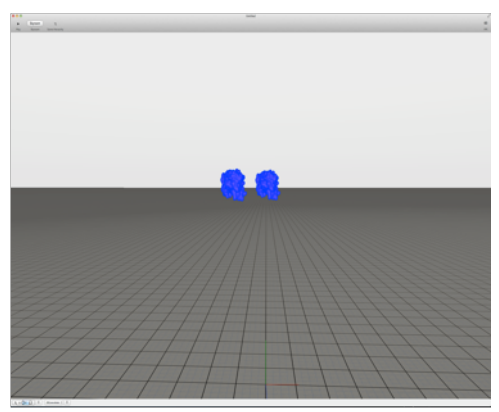

$\mathrm{F}$

Figure 5.2: Process of adding new (pre-defined) agents from the Lindsay Composer's Skyroom into the Simulation Environment. Here, we are adding a CheB agent into the environment by selecting the Skyroom from the Composer's Toolbar and then dragging in our agent into the environment.

\subsection{The Speed}

Time is valuable and our model takes note of this. A simple slider bar in our interface allows the user to control the speed of the simulation. The simulation starts off with time similar to the real world, however with a simple drag, the speed of the time can be increased. This is important as most computer simulations and models are used to quickly visualize and see the outcome of a targeted task. We implemented the time bar (Figure 5.1) to allow the user the choice to let the simulation run in real-world time or to increase the speed of time to see what should happen at specific time points. 


\subsection{Travelling Through the Layers}

One primary focus of this thesis was to build a multiscale model. Each view contains one particular layer of detail of our model. In all, there are 4 different layers making up the 4 different views in our model. The user has the ability to freely move around in real-time in the $3 \mathrm{D}$ space the simulation is situated in.

The simulation begins by looking at a simulation environment similar to a petri dish. Here, the user will select points on that environment where the stimuli should be and where the E. coli inoculation point is. In this scene, we can see two of the 4 views (Figure 5.5), as mentioned in Chapter 4.2.1, the first two views are a visual simulation of chemotaxis at the population level.

Moving into the next scene, it will trigger the transition from population to a single bacterium. In this scene, we are able to observe the specific patterns of movement by the bacterium via chemotaxis (Figure 6.4). The ability to move freely allows the user to follow the bacterium's movement path towards its goal, either away, or towards a stimuli.

The next scene in sequence is moving inside the bacterium. Once inside the bacterium, molecules (particles) are randomly wandering about inside the cell (Figure 5.3). Taking a closer look, we can observe the molecular details of the chemotaxis pathway. If one finds the bacterium space over crowded with molecules, one can turn off the molecules (particles) and in this instant, only the molecular details of the chemotaxis pathway will be shown (Figure 5.4 . 


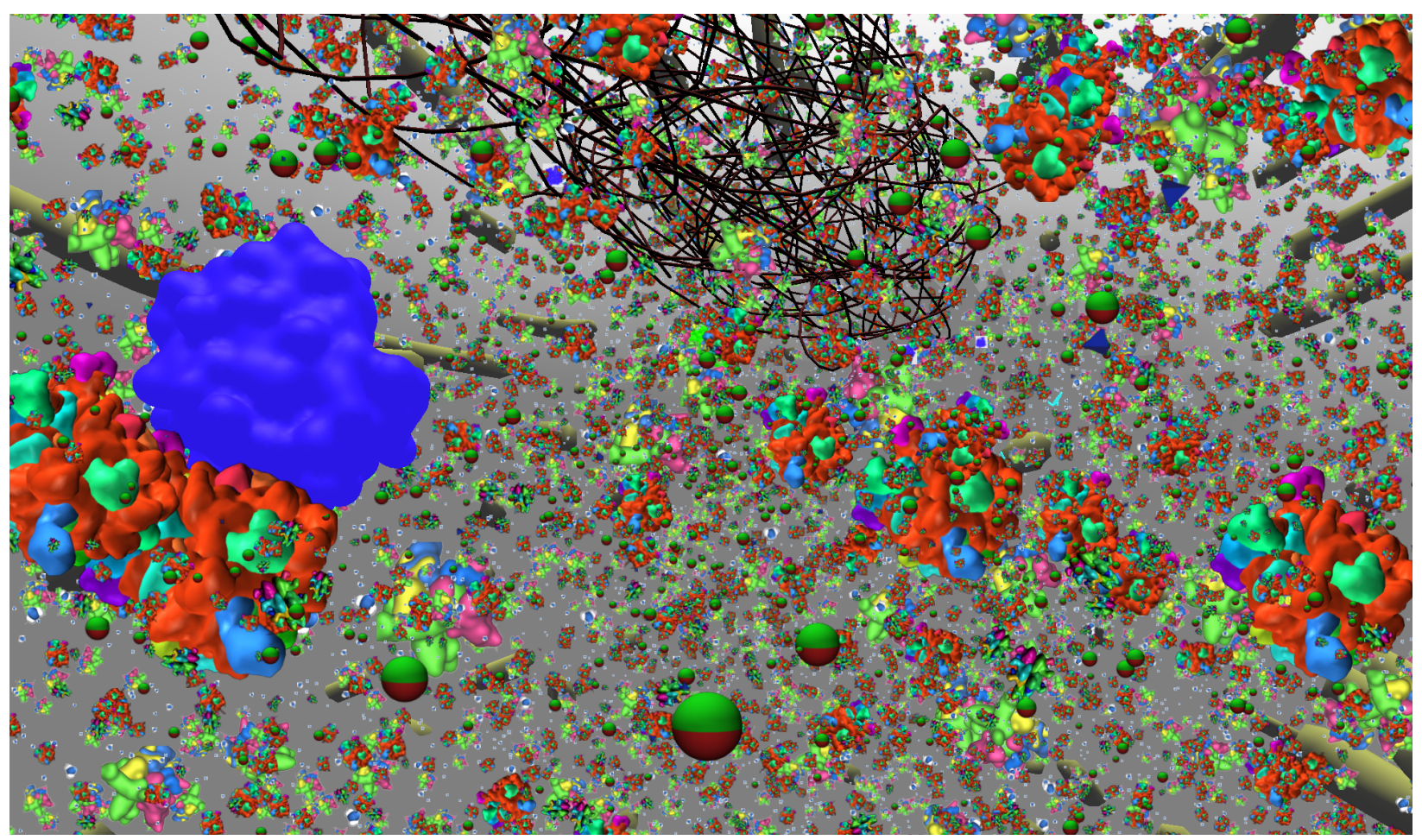

Figure 5.3: Molecular View - Inside the cell in the cytoplasm.

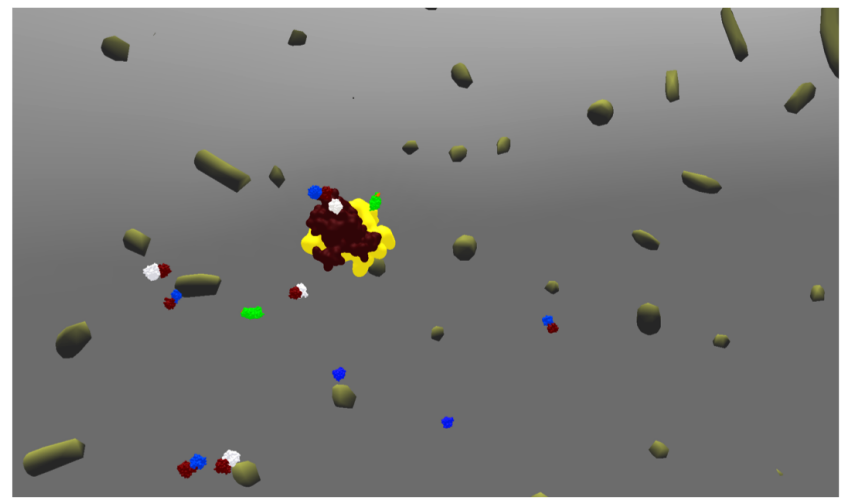

Particle System Off

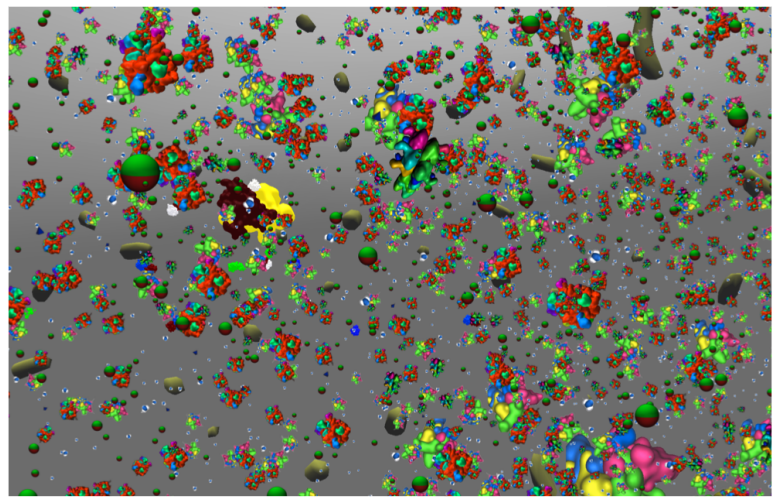

Particle System On

Figure 5.4: The molecular view with the particle system off and with the particle system on. 

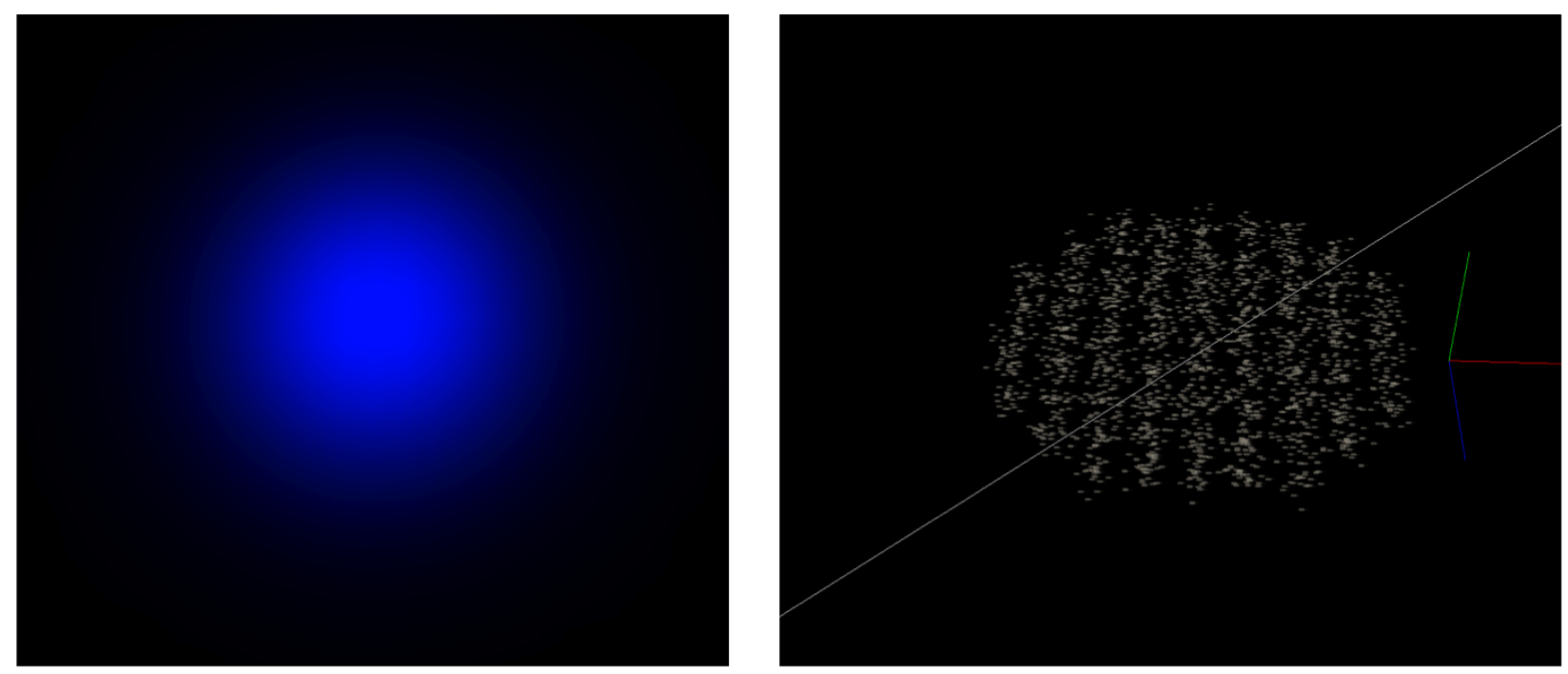

A - Layer One

B - Layer Two

Figure 5.5: A) Layer one (naked-eye view) of our multiscale model, shown is an single $E$. coli colony with an uniform/no stimulus environment. B) Layer two (colony view) of our multiscale model, rather than the $E$. coli gradient, we see the actual bacterium cells in the environment. 


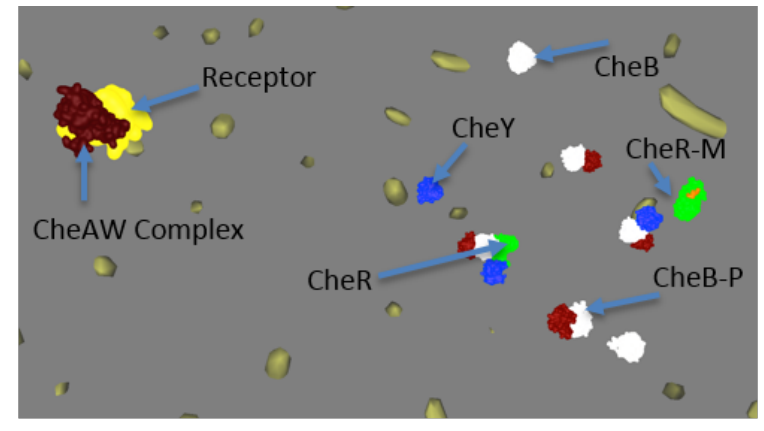

A

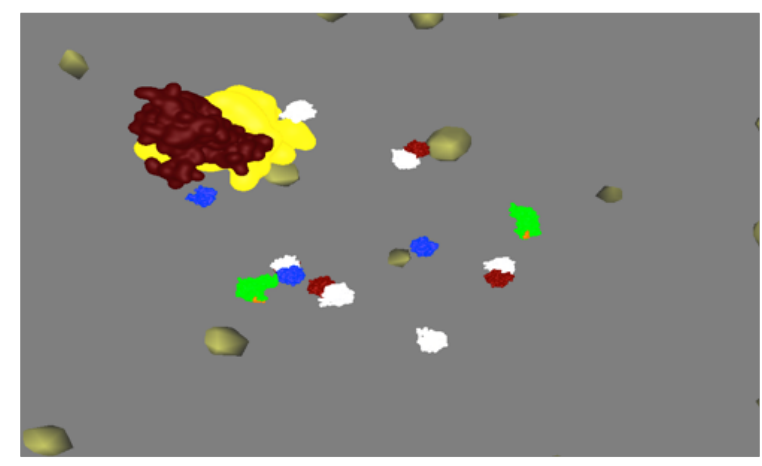

B

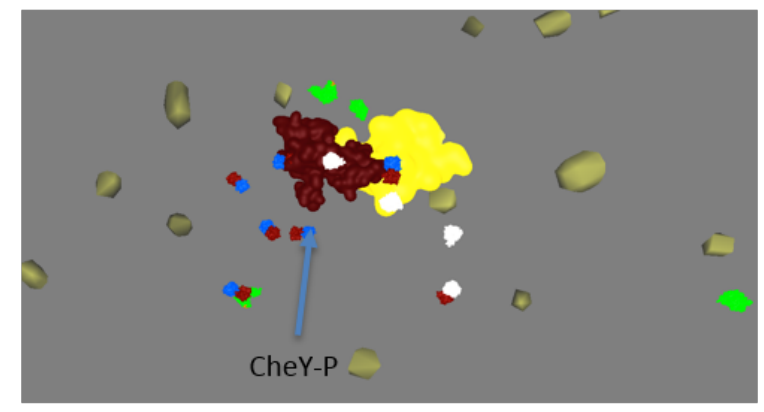

C

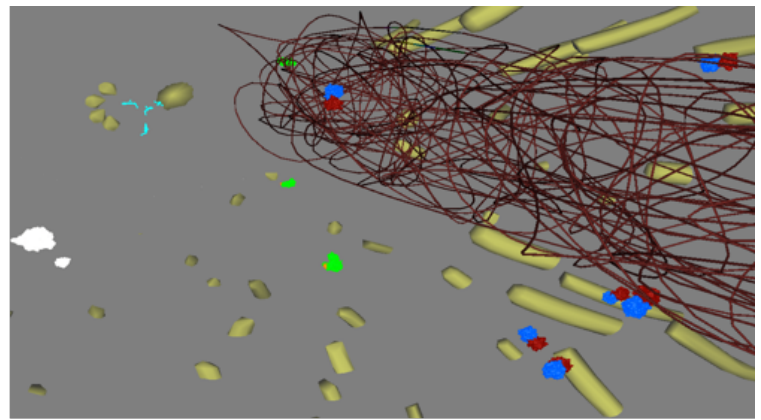

D

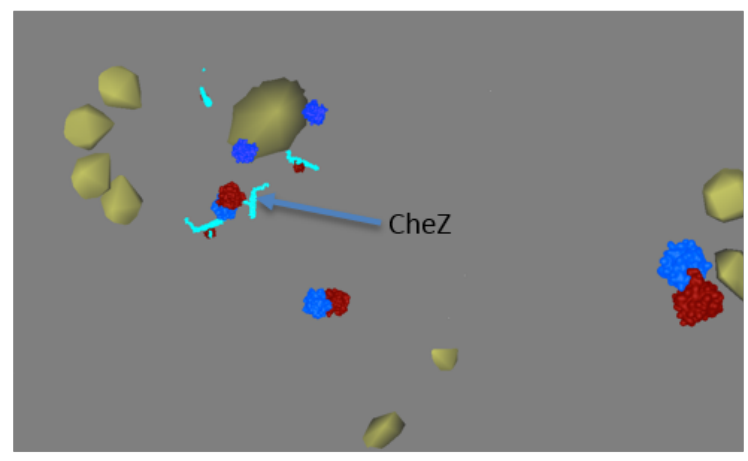

$\mathrm{E}$

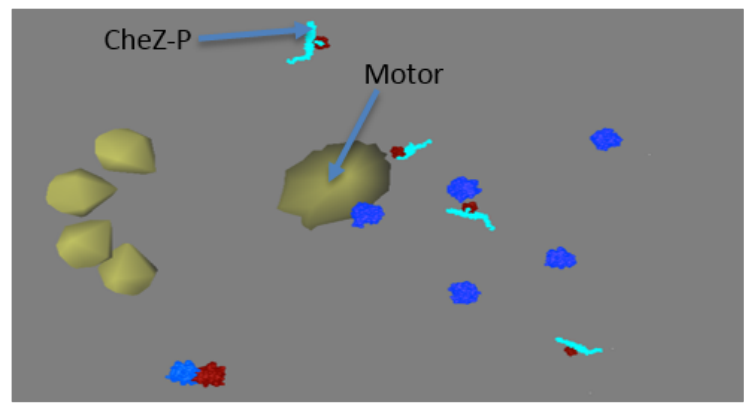

$\mathrm{F}$

Figure 5.6: Molecular details of the chemotaxis pathway with the particle systems turned off to reduce the density of elements as compared to Figure 4.8 . 


\section{Chapter 6}

\section{Results}

We have been using results from our simulation throughout the thesis to explain, describe, and showcase the implementation of our model. Starting with our naked-eye and colony view, we can see that in an environment such as an agar plate, with no external stimulus, chemotactic rings are formed (Figure $2.3 \mathrm{~B}, \mathrm{C}$ ), similar to a wet-lab experiment of E. coli growing in a tryptone soft agar plate.

When an attractant stimulus is placed within the environment, our simulated E. coli cells grows and moves towards the origin of attraction (Figure 2.2A). In Figure 6.1 - in the presence of an attractant in a real wetlab experiment - an E. coli colony grows towards the source of the attractant. The same behavior observed in wet-lab experiments (Figure 6.1A, 6.2 is reflected in our model. Similarly, with a repellent stimulus the colony grows away from the repelling source (Figure 2.2B). To illustrate the interactivity on our naked-eye and colony view, we can constantly add in new points of origins for the stimulus to react to. Each new stimulus addition causes a reaction with our E. coli colony. For example, consider Figure 6.3 of a constant addition of an attractant, our E. coli colony will follow the gradient and move towards the higher concentration of attractant.

In our individual view, we can see an E. coli bacterium following a gradient or performing a random walk in the absence of a stimulus (Figure 6.4. Following the bacterium long enough, we can see that it will eventually reach the location of an attractant - or in the case of a repellent - we can clearly see the bacterium moving away.

In the molecular view, we have replicated the different interaction phases of the chemotaxis pathway. Figure 2.7 and Figure 2.8 illustrate this with a side-by-side comparison of the pathway diagrams and their replication in our agent-based model, where the agent be- 


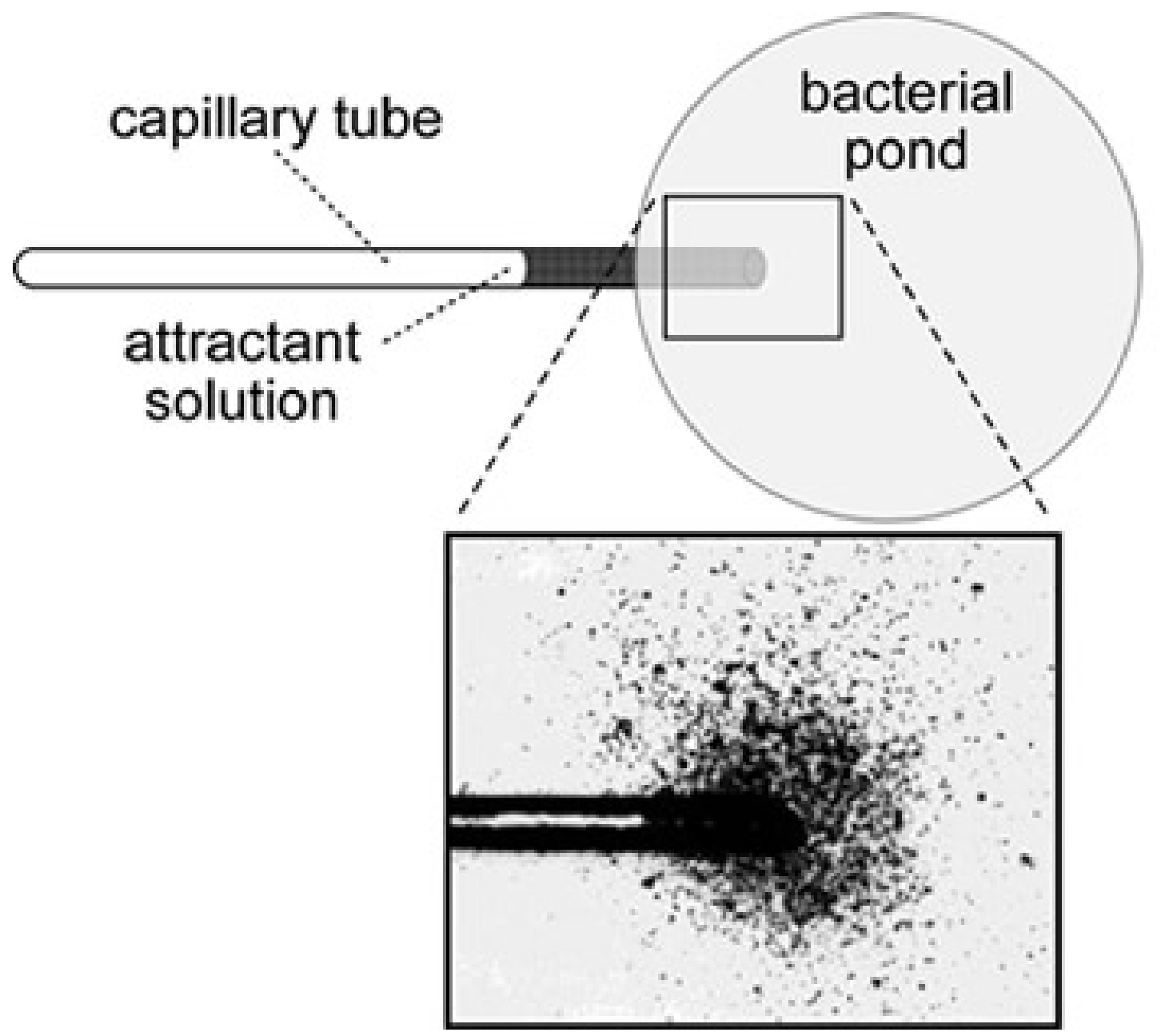

Figure 6.1: A capillary assay invented by W.Pfeffer in the 1880's. The test chemical diffuses from the capillary mouth, establishing a steep gradient that attracts bacteria to the entrance. The cells enter the capillary and are subsequently documented by colony counts. ${ }^{1}$

haviours are driven by short code scripts, shown by Algorithms $3,4,5$ and 6 .

\subsection{Multiscale and Interaction Interface}

One of the distinguishing aspects of our model is a real-time multiscale approach. As previously mentioned in Chapter 5.5 , we are able to travel through our simulation environment and transition between our implemented views as described in Chapter 4.2 (Figure 0.1).

\footnotetext{
${ }^{1}$ Printed with permission from Dr. John S. Parkinson Lab http://chemotaxis.biology.utah.edu/ Parkinson_Lab/projects/ecolichemotaxis/ecolichemotaxis.html
} 

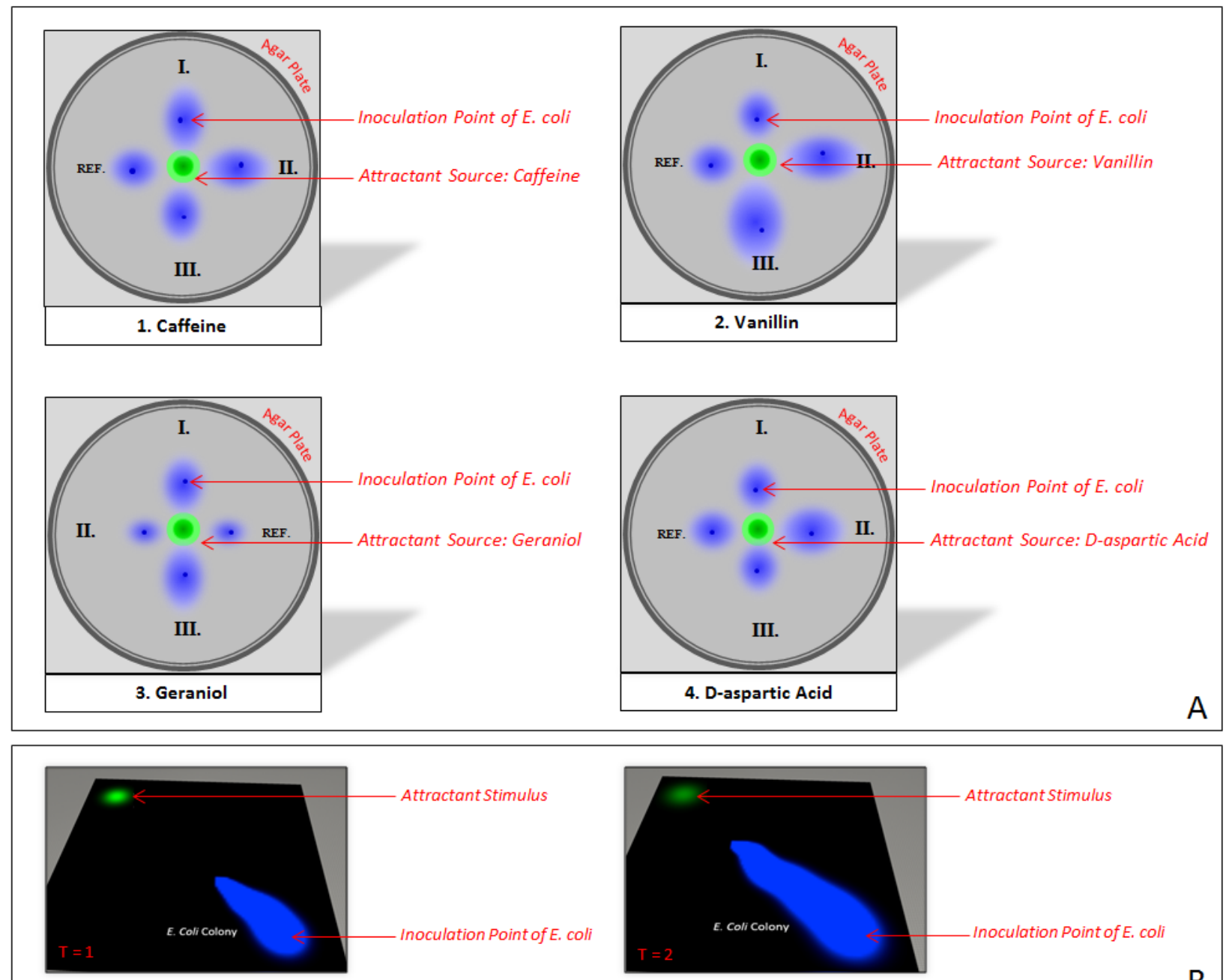

Figure 6.2: A) Figure redrawn from Figure 2b, from Team Goettingen, in the 2012 iGEM competition (http://2012.igem.org/Team:Goettingen/Notebook/Results). The figure shows the result of 4 strains of $E$. coli growing in the presence of an attractant, where one can see the chemotactic ring formation and the general growth direction of the $E$. coli in the presence of an attractant stimulus. B) Movement of the E. coli colony (blue) towards an attractant over two time steps $(\mathrm{T}=1, \mathrm{~T}=2)$ in our model. 


\begin{tabular}{|c|c|}
\hline Representation & Color \\
\hline E.Coli & Blue \\
\hline Attractant Stimulus & Green \\
\hline
\end{tabular}
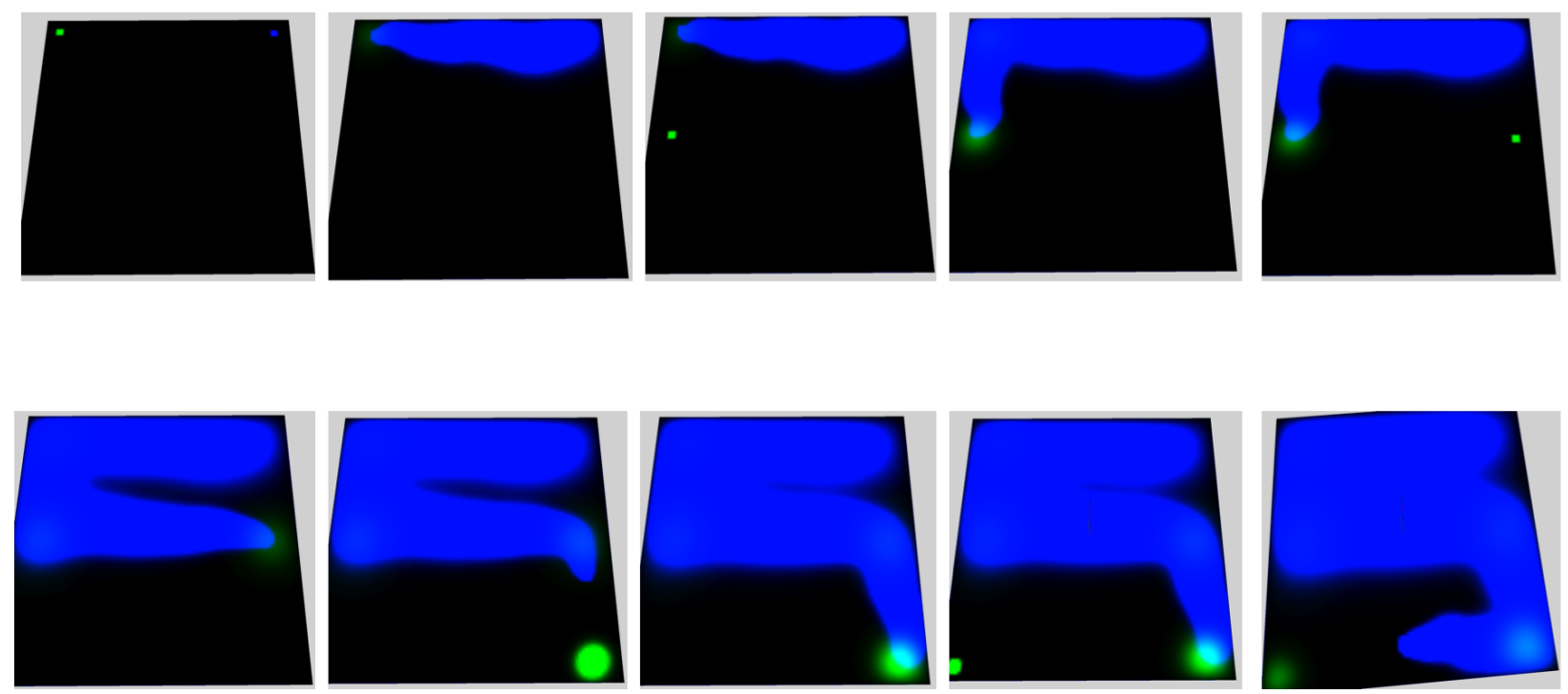

Figure 6.3: Continuous addition of attractant stimulus in different locations. With each new addition, the E. coli moves towards the new high concentration of the attractant. 

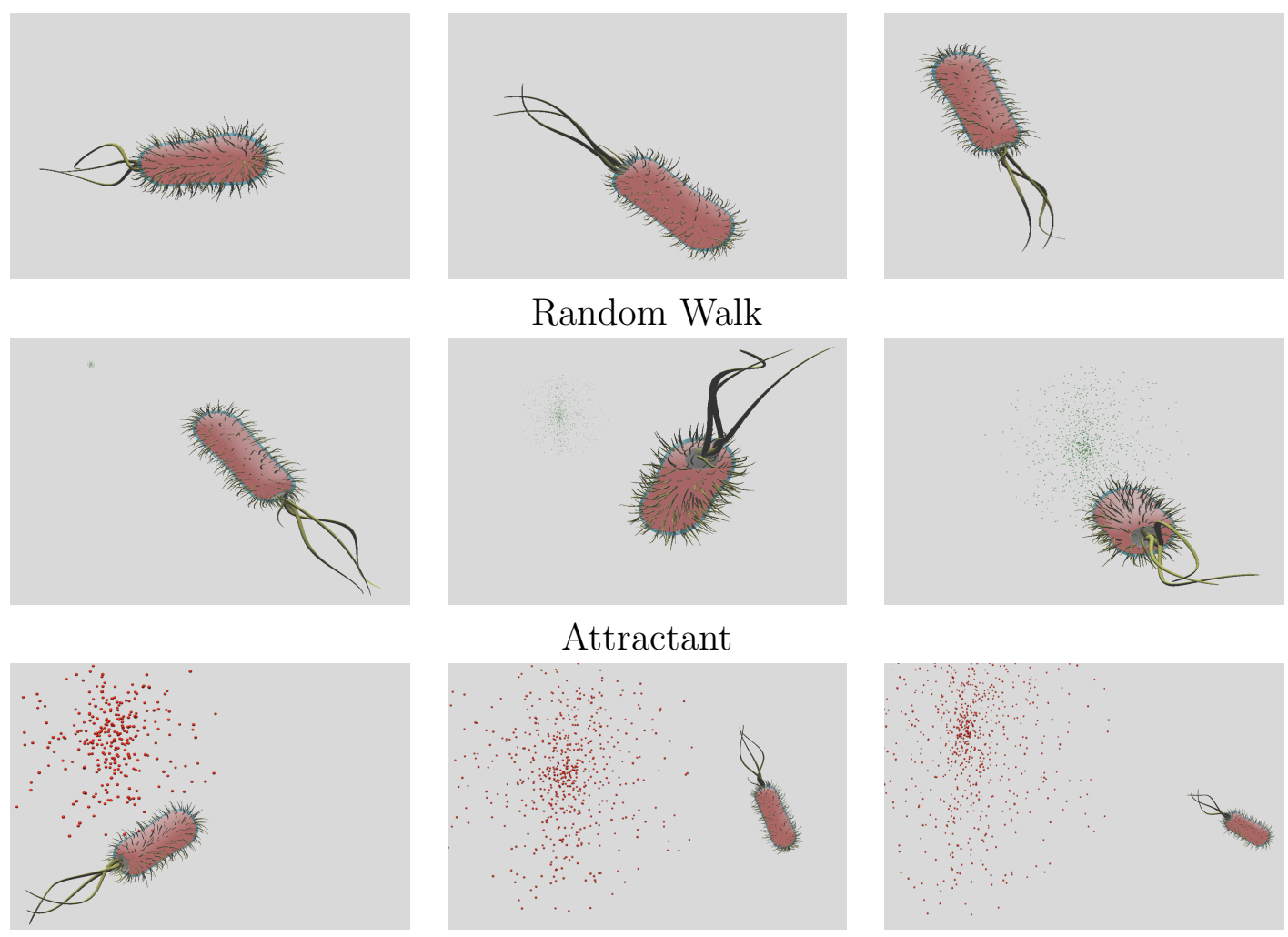

Repellent

Figure 6.4: E. coli as a single agent in agar environment. 


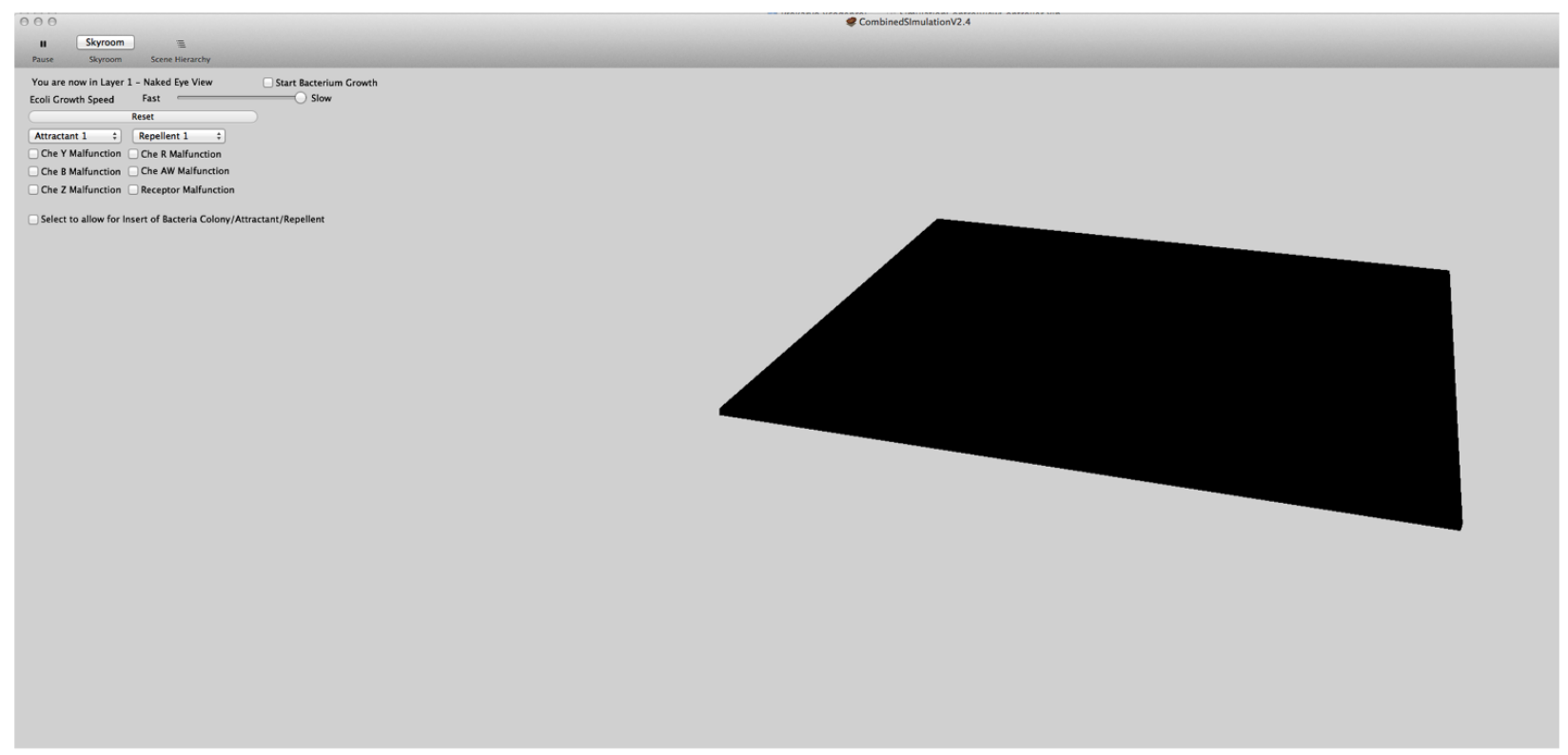

Figure 6.5: Embedded Interface directly into the LINDSAY Composer Simulation Environment.

To clearly distinguish between which view we are currently in while traveling through our simulation, we have embedded an interactive interface directly into the simulation area (Figure 6.5). As Figure 6.6 demonstrates, each view has its own labelled interface, and each view has a similar interface with unique interaction components specific to a view.

\subsubsection{Protein Interaction}

The interaction is a simple on-off switch of the protein itself. A protein that is in the state of "Malfunction", in the context of our model is the same as saying the protein is "Disabled" or "Off". In Chapter 5, the effects of the protein interaction are explained, the protein will either function or not function properly. Here, we illustrate sample results of such effects.

In Figure 6.7, we can see the control interface for switching CheAW complex on and off. The complex naturally switches between an active (on) and inactive (off) state due to the excitation and adaptation process of chemotaxis as explained in Chapter 2.3.1. In Figure 6.8, we see the difference between the complex always on and always off. When the complex is always on, we can see that the proteins $\mathrm{CheY}$ and CheB are phosphorylated. 
Assuming normal functionality of all other proteins, we will see a constant phosphorylation of CheY and CheB. However, if the complex is always off, there will eventually be no more phosphorylated proteins of $\mathrm{CheY}$ and CheB.

Another example, is CheZ, which would cause the opposite effect that CheAW complex has on CheY. If CheZ is disabled (off), there would be no protein to dephosphorylate the phosphorylated CheY (CheY-P). Leading to an eventual 100\% bias towards CheY-P concentration as we can see in Figure 6.9,

\subsubsection{Multiscale Interaction}

Interactions that occur on any view in the simulation is directly transferred into the next view when we transition between the views. As Figure 6.10 shows, we disable the receptor protein in our naked-eye view, and as we transition through our other views, we can see that the receptor protein continues to be disabled. This is evident not only by the interface embedded into the simulation, but also by how the simulation is actually executing.

In our example of a disabled receptor protein, this would lead to the always-off case of our CheAW complex. An always off CheAW complex, would eventually lead to a $100 \%$ bias of CheY. With only CheY inside the bacterium, this leads to a constant run regardless of conditions in the environment. In the naked-eye view (Figure 6.11), we can see this effect by how the $E$. coli ignores the conditions in its environment. In the individual view (Figure 6.12), we can see that the cell just keeps running without ever changing its direction (tumble), and in the molecular view (Figure 6.8), we can see that there are no CheY-P or CheB-P proteins. 


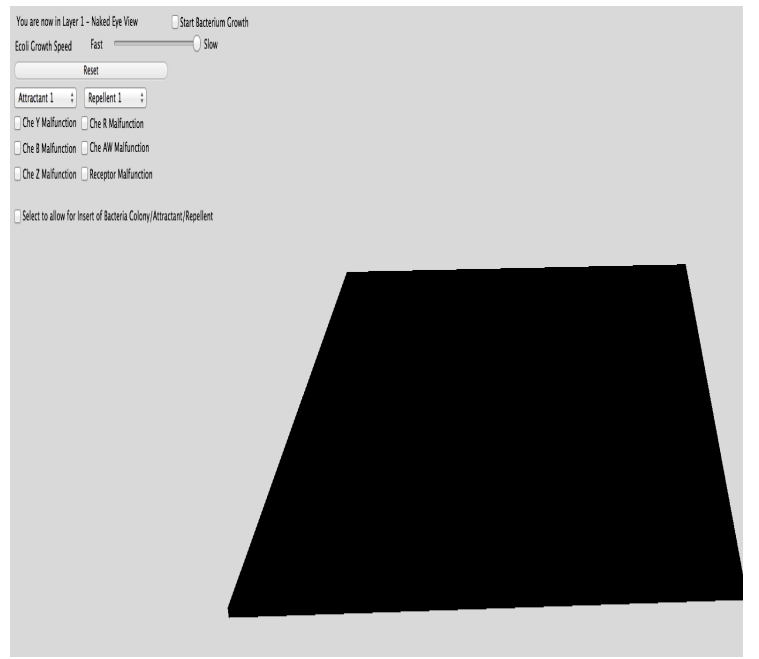

Naked-eye View Interface

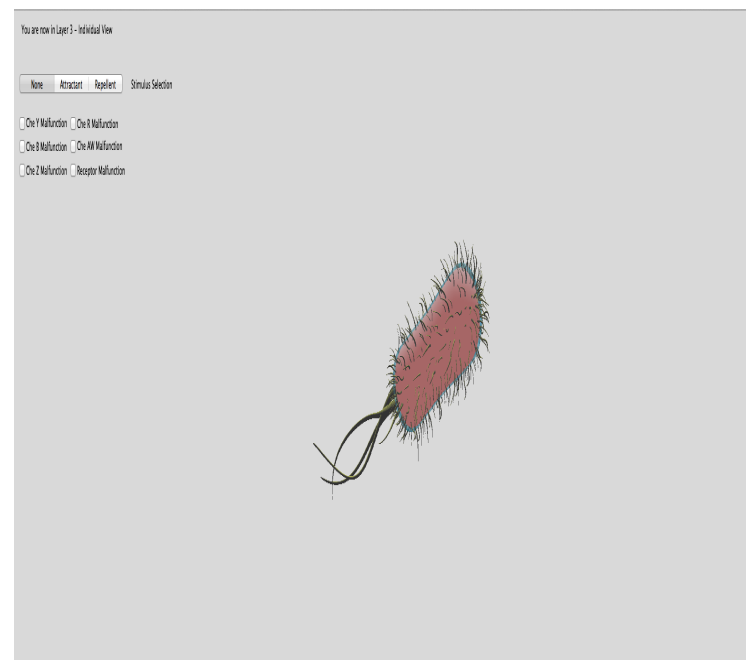

Individual View Interface

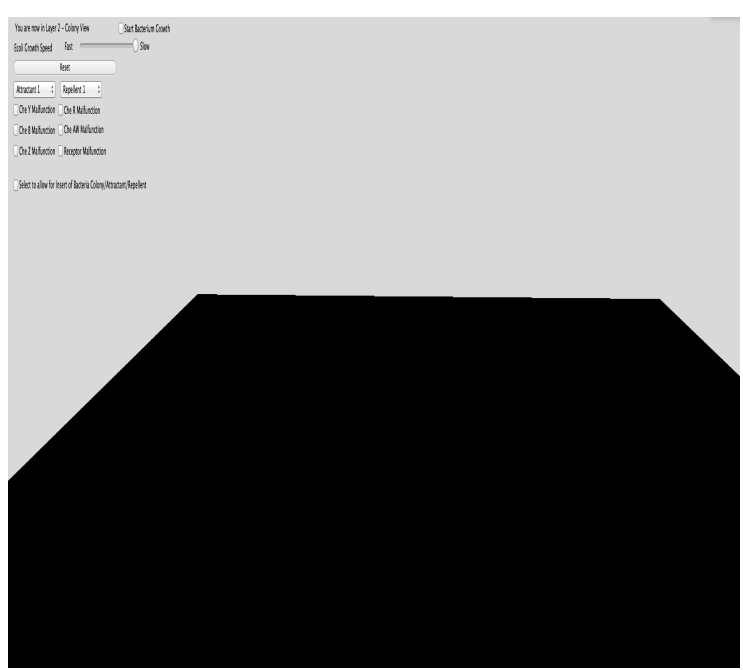

Colony View Interface

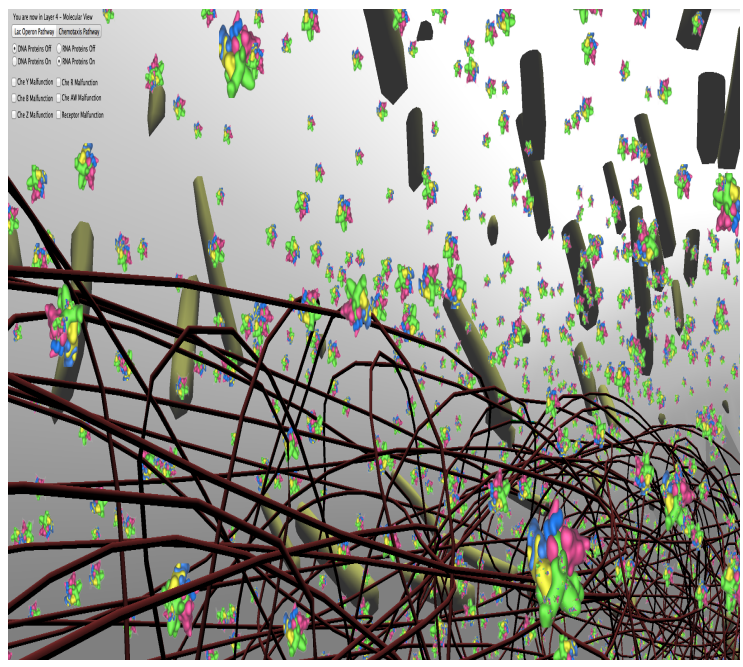

Molecular View Interface

Figure 6.6: Snapshots of the four interfaces for each of our views within the multiscale $E$. coli model.

\section{Che AW Malfunction}

\section{A}

\section{$\checkmark$ Che AW Malfunction}

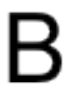

Figure 6.7: A) CheAW in the default state (On). B) CheAW in disabled (Malfunction) state (Off). 

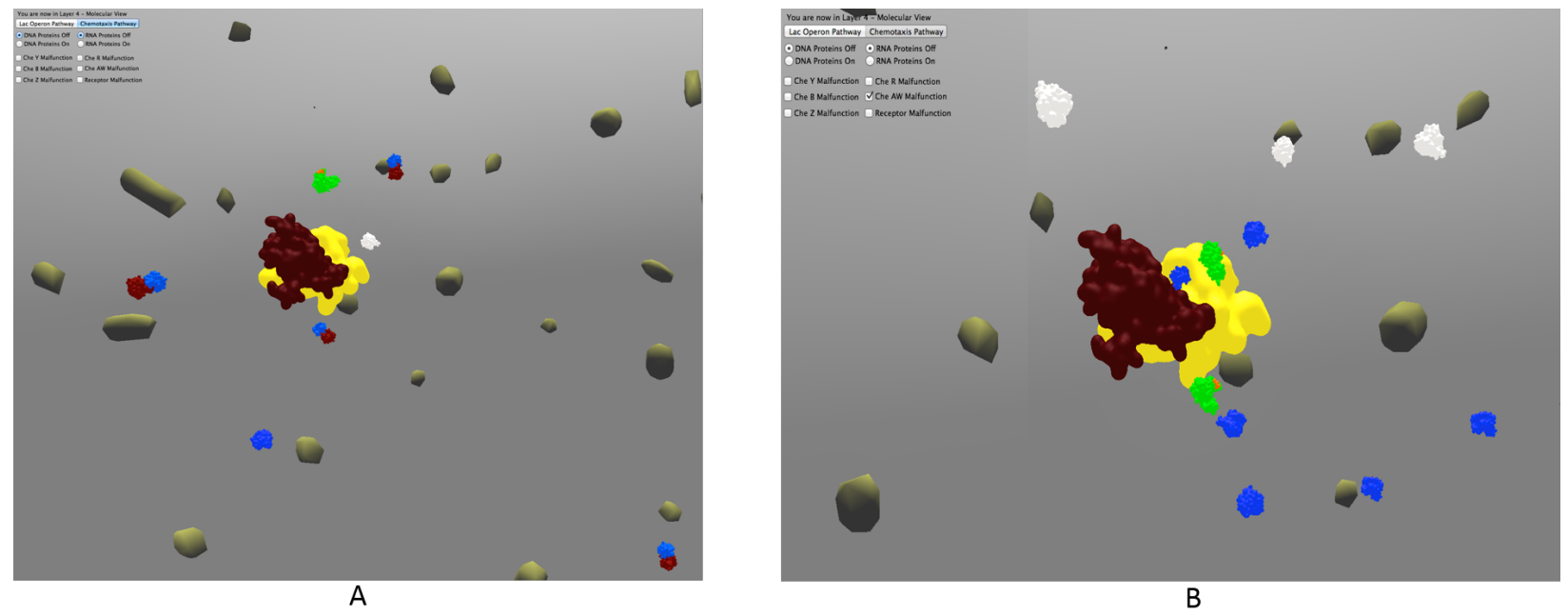

Figure 6.8: A) With CheAW active, we can see normal phosphorylation events do occur for CheB and CheY resulting in CheB-P and CheY-P. B) With CheAW inactive, no phosphorylation occurs.

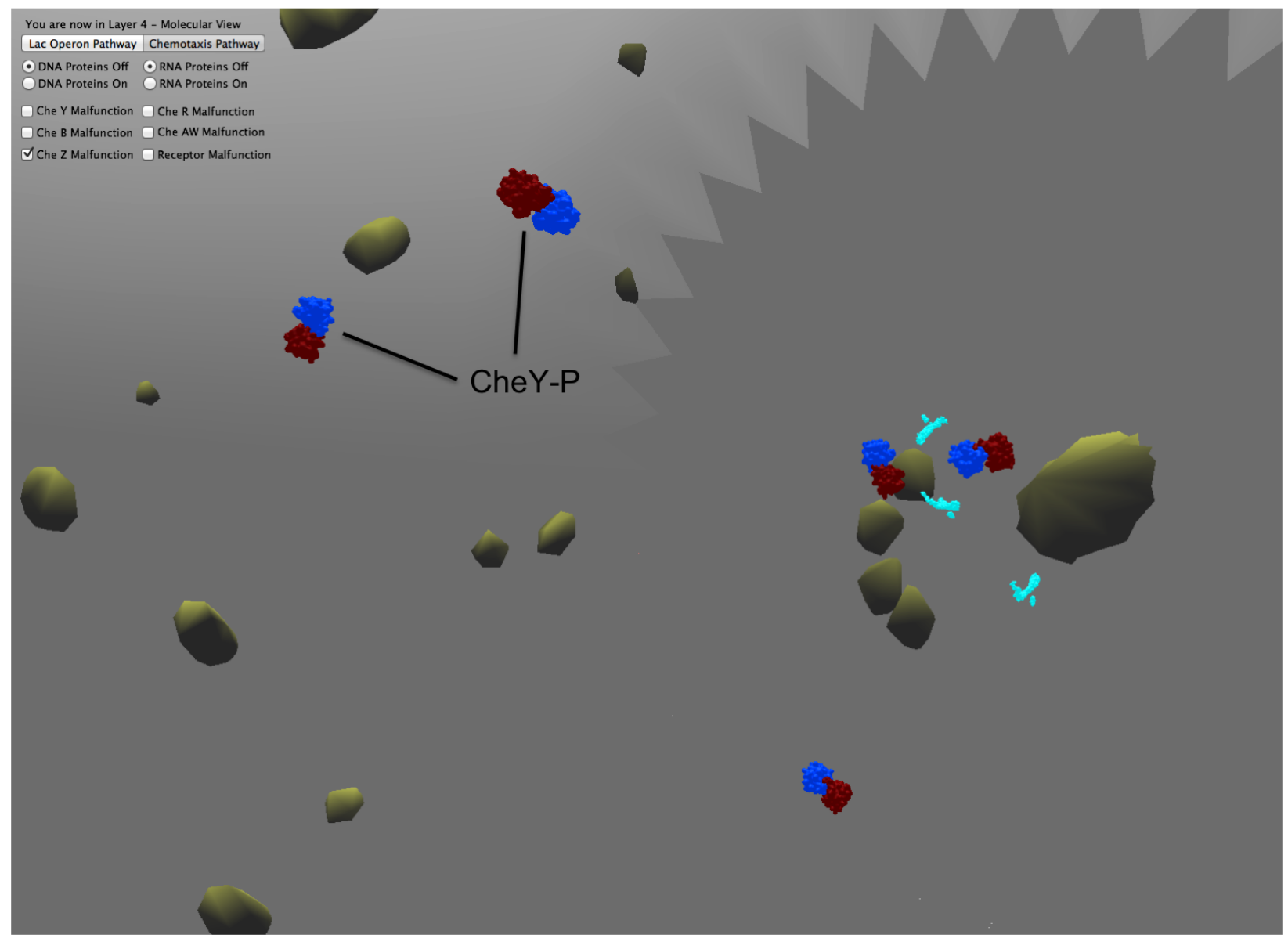

Figure 6.9: With CheZ disabled, no phosphorylation can occur, leading to all proteins such as CheY to be phosphorylated. 


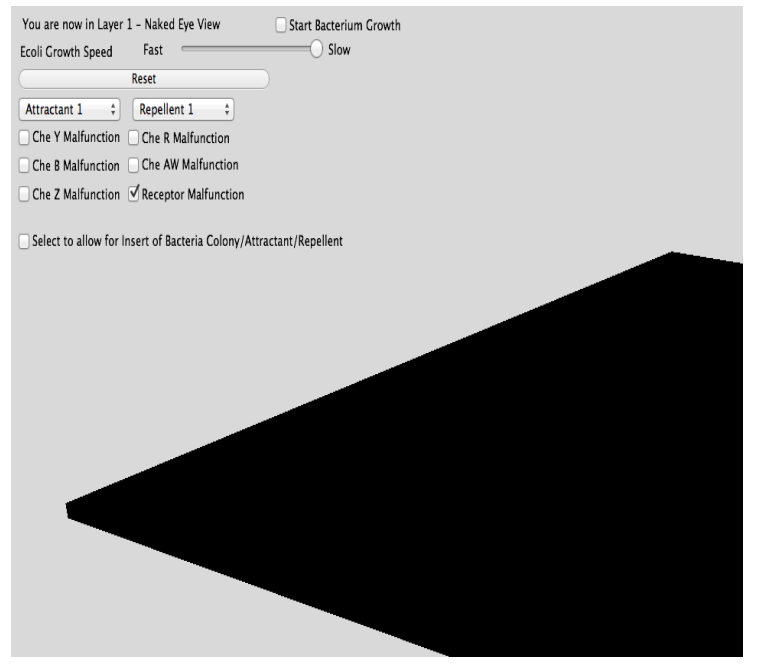

Naked-eye View Interface

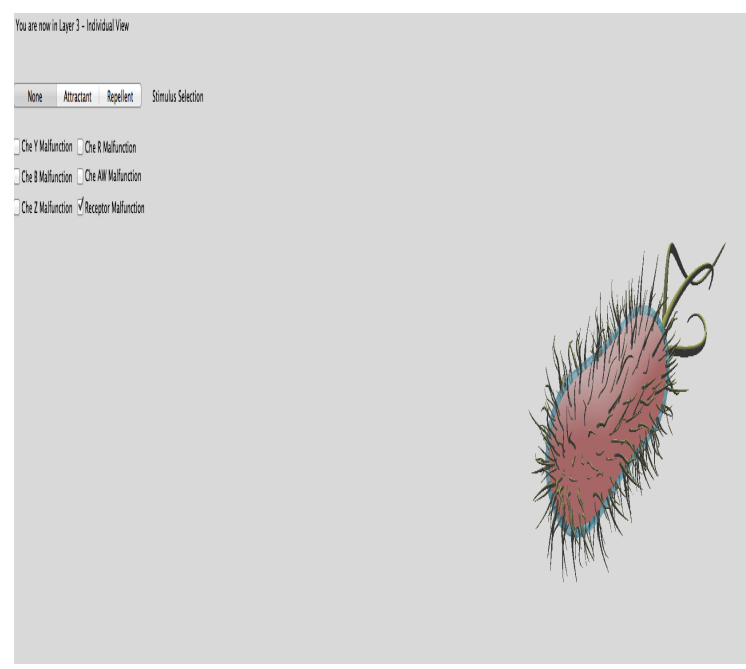

Individual View Interface

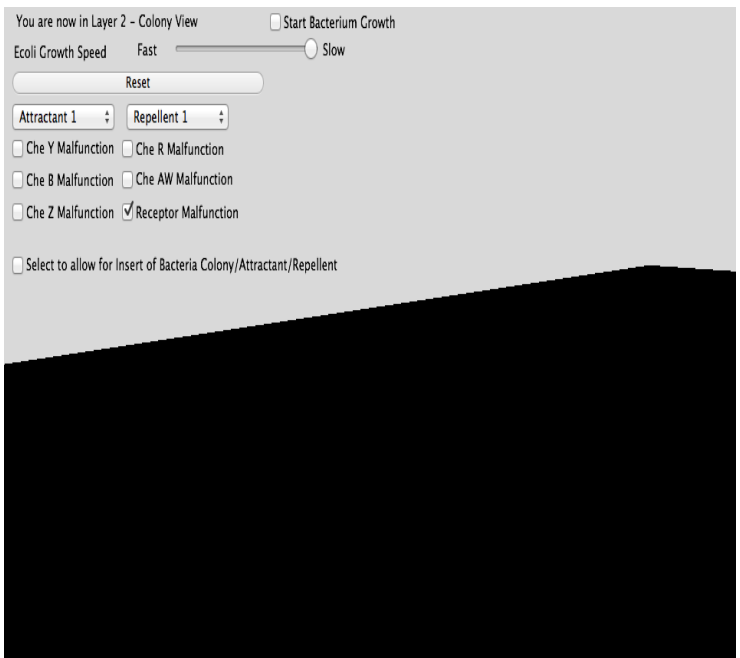

Colony View Interface

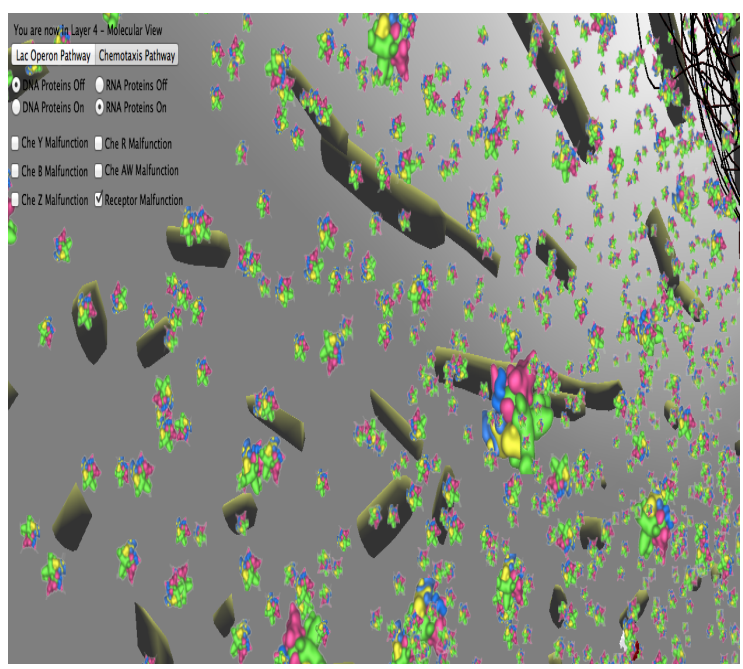

Molecular View Interface

Figure 6.10: Disabled receptor agent maintained through Eye (view 1) to Molecular (view 4) transitions in the multiscale model. 

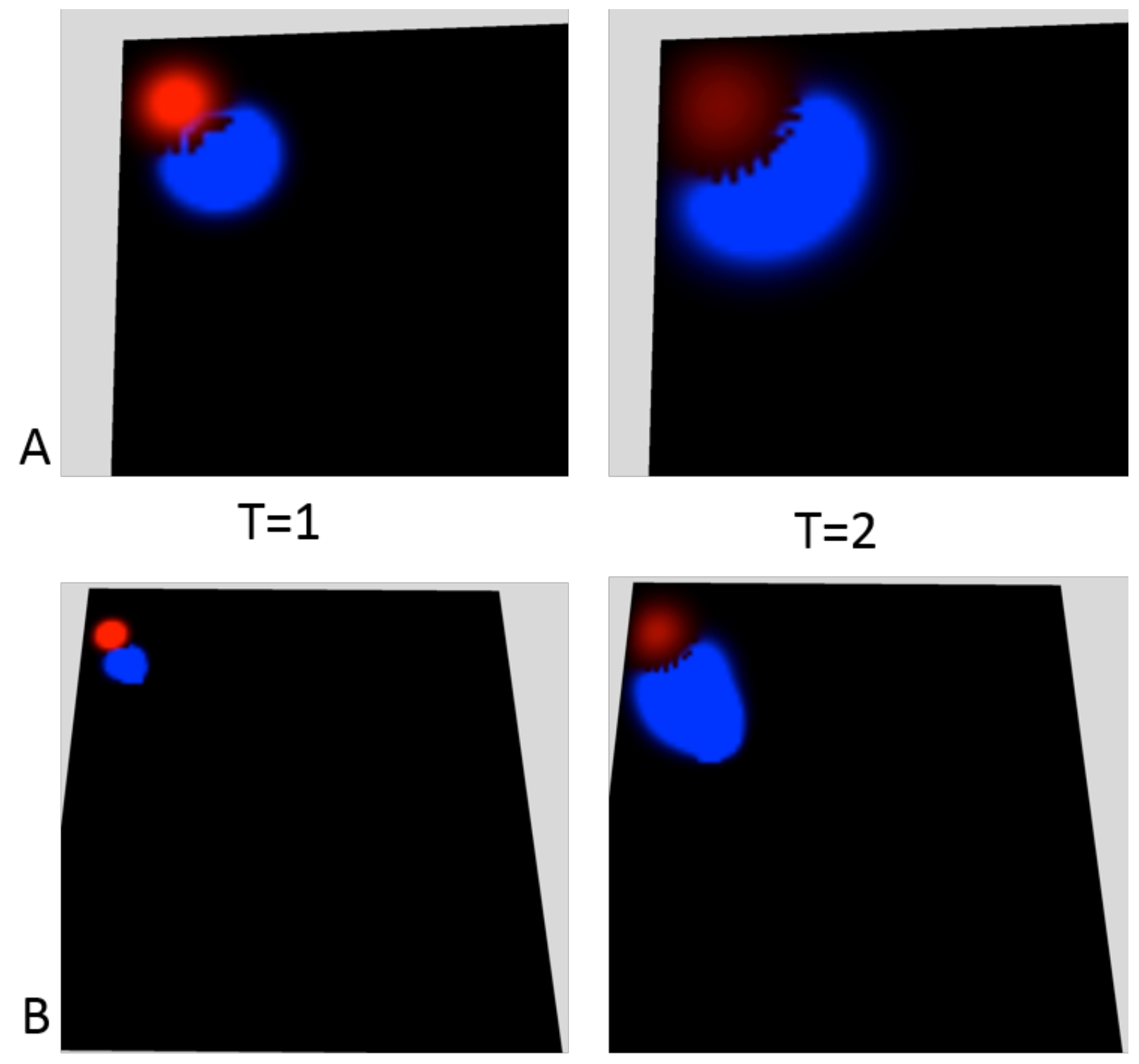

Figure 6.11: A) Disabled receptor, we can see how the E. coli colony ignores the repellent stimulus beside it as it does not distinctly run away as compared with B) where the receptor is functional and we can see that the E. coli colony moves away from the repellent. 

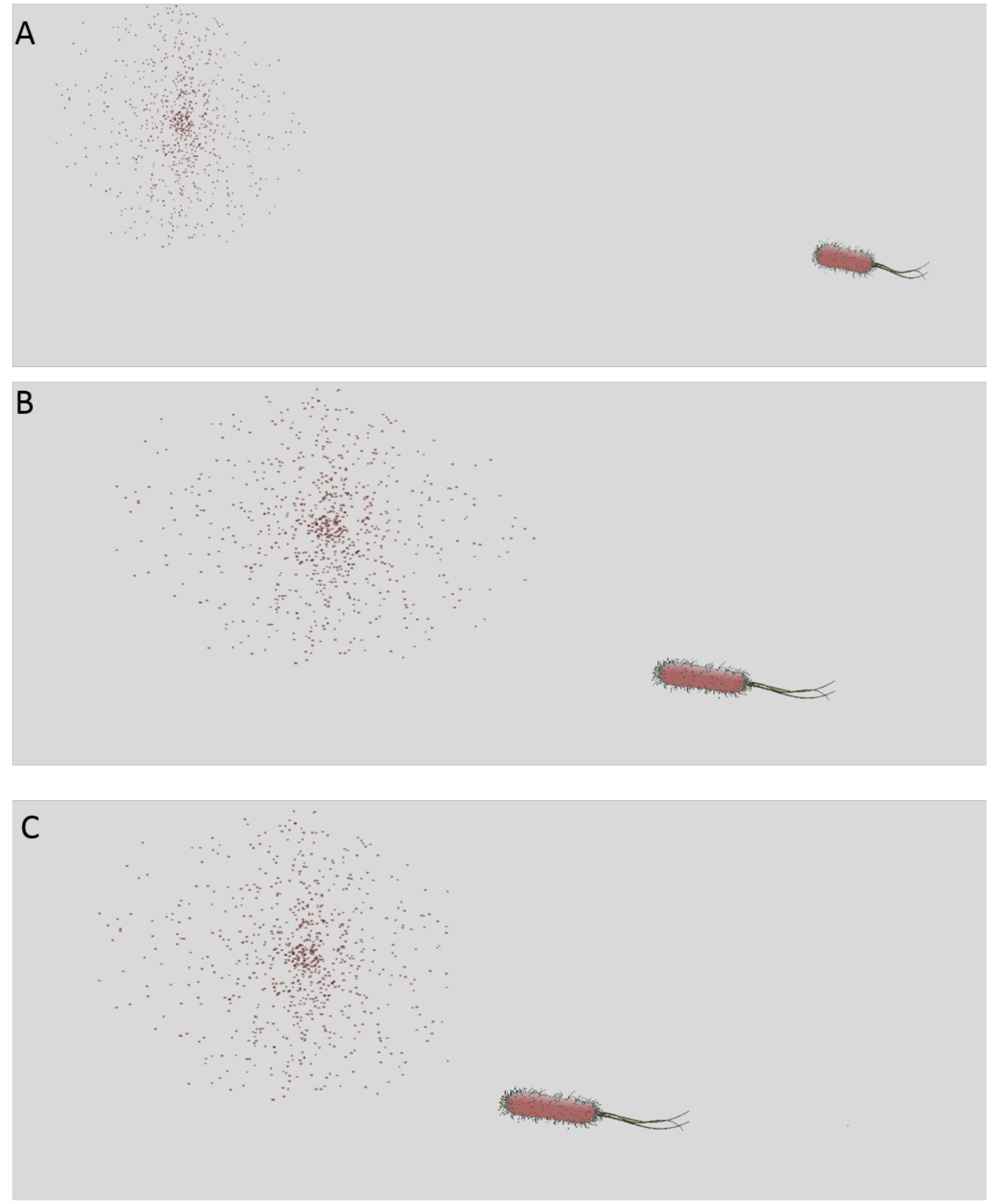

Figure 6.12: Bacterium running straight into the repellent. With the receptor disabled, there is no signal to phosphorylate the CheY. This leads to a higher concentration of CheY which suppresses tumbling. 


\section{Chapter 7}

\section{Conclusion}

As one of the most studied organisms, E. coli is an ideal model organism due in part to the vast amount of information available on this bacterium. Movement is as important as any other aspect of a living organism, such as metabolism. Here, we have incorporated the

chemotaxis pathway responsible for the movement patterns of $E$. coli in the presence and absence of a stimulus.

The goal of this thesis was to build an interactive, 3D, multiscale, and real-time model of chemotaxis to understand the complex interactions that drive the chemotactic behaviour. This allowed for the visualization, observation, and analysis of how changes on the molecular level could affect the population level and vice versa.

Successful implementation of this model utilized three different modelling techniques: mathematical (equation based), agent-based, and multiscale modelling. These three techniques are presented together to create a tool for capturing cellular processes in a visual, adaptive, and interactive way.

In conclusion, equations were used to capture the system dynamics and cellular processes with a high number of interactive entities, while the agent based modeling system captures the finite details of the cellular processes when the number of interactive entities are low. Together with the multiscale modelling technique, we have created a tool that allows for the exploration of the chemotactic movement of bacteria from the population aspect to the molecular details.

In summary, we have successfully implemented our outlined objectives and features mentioned in Chapter 1.3. The objectives to implement the chemotaxis pathway and multiscale aspect to the model was successfully completed as we have presented throughout the the- 
sis. Along with these objectives, we also presented that each feature was also successfully implemented. We have created a biologically accurate, interactive and robust, and visual multiscale model of chemotaxis in Escherichia coli .

The successful implementation of this tool came with its set of challenges highlighted in Chapter 4. The obstacles were solved and a successful implementation of the multiscale chemotaxis model was accomplished. With the power of imagination and evolving technologies and the will to explore and succeed, we can make visible what previously only existed in equations and words. 


\section{Chapter 8}

\section{Future Work}

Not long ago, most students learned through the usage of textbooks and artistic images. In today's society, students have resources such as computer simulations, videos, and animations, improving the effectiveness of student learning even more. Students have the ability to learn anything from simple systems to complex ones such as the cellular process inside a cell. As educators and researchers, technology allows for a different and unique approach to sharing knowledge. Advancements in the field of technology are changing the way we learn, educate, and perform research.

This virtual E. coli model provides an alternative method to learning and studying the cellular processes. Here, I put forward some suggestions on future extensions that could improve upon the current model.

- A Pathway Integration Interface Currently, the model supports two pathways: the previously built lac operon and now the chemotaxis pathway. However, each of these implementations required a lot of work behind the scenes in modifying and adding code. A pathway integration interface could be developed such that some specific information is provided, and the model can dynamically incorporate a basic functional pathway based on the information provided.

- Mobile Devices The current model functions solely on an Apple desktop. Being able to expand the current model into functioning with mobile devices such as iPads and iPhones would make the system more readily accessible by users. This accessibility could then be used in classroom settings to complement the learning of cellular processes in Biology. 
- Incorporation of Other Simulation Environments Currently, at the population layer, the stimulus and E. coli need to be placed within the boundaries of a defined simulation environment representing a petri-dish, the removal of this limitation would greatly increase the ability to incorporate this model into existing software such as the LINDSAY Virtual Human app, now widely available on the app store (Zygote 3D Anatomy Atlas \& Dissection Lab LINDSAY Virtual Human).

- Virtual Reality The latest trend of technology now is virtual reality. Although not widely used in society yet, this is the direction we are heading towards. Adaptations of virtual reality include but are not limited to the Leap Controller [38], Myo Armband [58], the Oculus Rift [50], and other similar emerging technology products. 


\section{Bibliography}

[1] Robert G Abbott, Stephanie Forrest, and Kenneth J Pienta. Simulating the Hallmarks of Cancer. Artificial life, 12(4):617-634, October 2006.

[2] J Adler. Chemotaxis in bacteria. Science, 153(3737):708-715, 1966.

[3] J Adler. Effect of amino acids and oxygen on chemotaxis in Escherichia coli. Journal of bacteriology, 92(1):121-129, 1966.

[4] J Adler. Chemoreceptors in bacteria. Science, 166(3913):1588-1597, 1969.

[5] J Adler. A method for measuring chemotaxis and use of the method to determine optimum conditions for chemotaxis by Escherichia coli. Journal of General Microbiology, 74:77-91, 1973.

[6] J Adler, G L Hazelbauer, and M M Dahl. Chemotaxis toward sugars in Escherichia coli. Journal of Bacteriology, 115(3):824, 1973.

[7] Uri Alon. An Introduction to Systems Biology: Design Principles of Biological Circuits. Chapman and Hall/CRC, 2007.

[8] Gary An. In silico experiments of existing and hypothetical cytokine-directed clinical trials using agent-based modeling. Critical care medicine, 32(10):2050-60, October 2004.

[9] S S Andrews and D Bray. Stochastic simulation of chemical reactions with spatial resolution and single molecule detail. Physical Biology, 1(3):137-151, September 2004.

[10] Autodesk Inc. Autodesk Education Community. http://students.autodesk.com/, 2012. [Online; accessed 28-June-2012]. 
[11] Autodesk Inc. Maya - 3D Animation Software (version 2012). http://usa.autodesk. com/maya/, 2012.

[12] N Barkai and S Leibler. Robustness in simple biochemical networks. Nature, 387(6636):913-917, 1997.

[13] H C Berg. E. coli in Motion. Springer, 2004.

[14] M Berry. Would-be worlds: How simulation is changing the frontiers of science Casti,JL. Nature, 385(6611):33-33, 1997.

[15] BioVisions Project - Harvard University. Inner life of the cell. http://biovisions. mcb.harvard.edu/, 2007.

[16] Blender.ORG. Blender - Open source 3D content creation suit (version 2.49b). http: //www.blender.org/, 2012.

[17] Georgiy V Bobashev, D Michael Goedecke, and Joshua M Epstein. A Hybrid Epidemic Model: Combining The Advantages Of Agent-Based And Equation-Based Approaches. In 2007 Winter Simulation Conference, pages 1532-1537. IEEE, 2007.

[18] Eric Bonabeau. Agent-based modeling: methods and techniques for simulating human systems. Proceedings of the National Academy of Sciences of the United States of America, 99 Suppl 3(90003):7280-7287, May 2002.

[19] D Bray and R B Bourret. Computer analysis of the binding reactions leading to a transmembrane receptor-linked multiprotein complex involved in bacterial chemotaxis. Molecular biology of the cell, 6(10):1367-1380, 1995.

[20] D Bray, R B Bourret, and M I Simon. Computer simulation of the phosphorylation cascade controlling bacterial chemotaxis. Molecular Biology of the Cell, 4(5):469-482, 1993. 
[21] D Bray, M D Levin, and C J Morton-Firth. Receptor clustering as a cellular mechanism to control sensitivity. Nature, 393(6680):85-88, 1998.

[22] Dennis Bray, Matthew D Levin, and Karen Lipkow. The chemotactic behavior of computer-based surrogate bacteria. Current Biology, 17(1):12-19, 2007.

[23] Levin M.D Bray, D and K Lipkow. The chemotactic behavior of computer-based surrogate bacteria. Current Biology, 17:12-19, 2007.

[24] Arancha Casal, Cenk Sumen, Timothy E Reddy, Mark S Alber, and Peter P Lee. Agentbased modeling of the context dependency in $\mathrm{T}$ cell recognition. Journal of theoretical biology, 236(4):376-391, October 2005.

[25] J C Dallon and H G Othmer. A discrete cell model with adaptive signalling for aggregation of Dictyostelium discoideum. Philosophical Transactions of the Royal Society B-Biological Sciences, 352(1351):391-417, 1997.

[26] T Emonet, C M Macal, M J North, and C E Wickersham. AgentCell: a digital single-cell assay for bacterial chemotaxis. Bioinformatics, 21(11):2714-2721, 2005.

[27] Afshin Esmaeili. An artificial escherichia coli bacterium brought to life: A journey inside the cell. Master's thesis, University of Calgary, Department of Computer Science, 2012.

[28] P Feng, S D Weagant, M A Grant, and W Burkhardt. BAM: Enumeration of Escherichia coli and the Coliform Bacteria, September 2002.

[29] Chrisantha Fernando. Computer Models of bacterial Chemotaxis, March 2005.

[30] Benjamin Franz and Radek Erban. Hybrid Modelling of Individual Movement and Collective Behaviour. Dispersal, Individual Movement and Spatial Ecology: a Mathematical Perspective, 2071(Chapter 5):129-157, 2013. 
[31] Genetic Science Learning Center - University of Utah. Cells and scale. http://learn. genetics.utah.edu/content/cells/scale/, 2014.

[32] David S. Goodsell. The Machinery of Life. Springer New York, 2nd edition, 2009.

[33] D C Hauri and J Ross. A model of excitation and adaptation in bacterial chemotaxis. Biophysical journal, 68(2):708-722, 1995.

[34] C Jacob, S von Mammen, T Davison, A Sarraf-Shirazi, V Sarpe, A Esmaeili, D Phillips, I Yazdanbod, S Novakowski, S Steil, C Gingras, H Jamniczky, B Hallgrimsson, and B Wright. LINDSAY Virtual Human: Multi-scale, Agent-based, and Interactive. Springer, 2012.

[35] E F Keller and L A Segel. Traveling Bands of Chemotactic Bacteria - Theoretical Analysis. Journal of theoretical biology, 30(2):235-\&, 1971.

[36] B E Knox, P N Devreotes, A Goldbeter, and L A Segel. A molecular mechanism for sensory adaptation based on ligand-induced receptor modification. Proc Natl Acad Sci $U S A, 83(8): 2345-2349,1986$.

[37] J J Landsberg and R H Waring. A generalised model of forest productivity using simplified concepts of radiation-use efficiency, carbon balance and partitioning. Forest Ecology and Management, 95(3):209-228, 1997.

[38] Leap Motion Inc. Leap Motion. https://www.leapmotion.com/, 2014. [Online; accessed 28-Nov-2014].

[39] LearningRx. Learning types, 2015.

[40] M D Levin, C J Morton-Firth, and W N Abouhamad. Origins of individual swimming behavior in bacteria. Biophysical journal, 74(1):175-181, 1998. 
[41] K Lipkow, S S Andrews, and D Bray. Simulated diffusion of phosphorylated CheY through the cytoplasm of Escherichia coli. Journal of bacteriology, 187(1):45-53, 2005.

[42] D Longo, S M Peirce, T C Skalak, L Davidson, M Marsden, B Dzamba, and D W DeSimone. Multicellular computer simulation of morphogenesis: blastocoel roof thinning and matrix assembly in Xenopus laevis. Developmental Biology, 271(1):210-222, July 2004.

[43] C M Macal and M J North. Tutorial on agent-based modeling and simulation. In Simulation Conference, 2005 Proceedings of the Winter, pages 2-15. IEEE, 2005.

[44] B A Mello and Y Tu. Perfect and near-perfect adaptation in a model of bacterial chemotaxis. Biophysical journal, 84(5):2943-2956, 2003.

[45] S Mellouli, B Moulin, and G Mineau. Laying down the foundations of an agent modelling methodology for fault-tolerant multi-agent systems. Engineering Societies in the Agents World Iv, 3071:275-293, 2003.

[46] Michael Reimpell. OGRE Blender Exporter. http://www.ogre3d.org/tikiwiki/ Blender+Exporter, 2012. [Online; accessed 28-June-2012].

[47] J Miller, M Parker, R B Bourret, and M C Giddings. An agent-based model of signal transduction in bacterial chemotaxis. PloS one, 5(5):e9454, 2010.

[48] MolecularMovies.org. mMaya - Molecular Maya Toolkit. http://www. molecularmovies.com/toolkit/, 2014.

[49] C J Morton-Firth, T S Shimizu, and D Bray. A free-energy-based stochastic simulation of the Tar receptor complex. Journal of molecular biology, 286(4):1059-1074, 1999.

[50] Oculus. Oculus Rift. https://www.oculus.com/dk2/, 2015. [Online; accessed 05-Mar2015]. 
[51] A A Patel, E T Gawlinski, S K Lemieux, and R A Gatenby. A cellular automaton model of early tumor growth and invasion: The effects of native tissue vascularity and increased anaerobic tumor metabolism. Journal of theoretical biology, 213(3):315-331, 2001.

[52] P R Patnaik. Robustness analysis of the E. coli chemosensory system to perturbations in chemoattractant concentrations. Bioinformatics, 23(7):875-881, 2007.

[53] Melissa Reneaux and Manoj Gopalakrishnan. From random to directed motion: Understanding chemotaxis in E. coli within a simplified model. arXiv.org, page 4583, December 2008.

[54] J E Segall, S M Block, and H C Berg. Temporal comparisons in bacterial chemotaxis. Proceedings of the National Academy of Sciences, 83(23):8987-8991, 1986.

[55] T S Shimizu, S V Aksenov, and D Bray. A spatially extended stochastic model of the bacterial chemotaxis signalling pathway. Journal of molecular biology, 329(2):291-309, 2003.

[56] V Sourjik. Receptor clustering and signal processing in E. coli chemotaxis. Trends in microbiology, 12(12):569-576, 2004.

[57] P A Spiro, J S Parkinson, and Hans G Othmer. A model of excitation and adaptation in bacterial chemotaxis. Proc Natl Acad Sci U S A, 94(14):7263-7268, 1997.

[58] ThalmicLabs Inc. Thalmic Labs. https://www.thalmic.com/en/myo/, 2014. [Online; accessed 28-Nov-2014].

[59] Torus Knot. OGRE (Version 1.8): Open Source 3D Graphics Engine. http://www. ogre3d.org, 2012.

[60] JMG Vilar, C C Guet, and S Leibler. Modeling network dynamics the lac operon, a case study. The Journal of cell biology, 161(3):471, 2003. 
[61] Sebastian von Mammen, Timothy Davison, Hamidreza Baghi, and Christian Jacob. Component-based networking for simulations in medical education. In The IEEE symposium on Computers and Communications, pages 975-979. IEEE, June 2010.

[62] G H Wadhams and J P Armitage. Making sense of it all: bacterial chemotaxis. Nature Reviews Molecular Cell Biology, 5(12):1024-1037, 2004.

[63] Wikipedia. Mathematical modelling — wikipedia, the free encyclopedia, 2014. [Online; accessed 16-Aug-2014].

[64] C Zimmer. Microcosm: E. coli and the new science of life. Vintage Books, 2009. 


\section{Appendix A}

\section{Lindsay Platform}

For realistic interactions of the cell's internal and external entities (such as proteins) the simulation environment has to be able to simulate and compute physical behaviours of entities within the cell. Thus, the cell behaviour can be mimicked realistically. Therefore, a suitable simulation environment has to be able to not only visualize the cell and its internal components, but also process collisions among entities in a realistic fashion. To achieve this, Lindsay Composer, a programming environment with built-in physics and high-end graphics for rendering in real-time, was used [34].

\section{A.1 Lindsay Composer}

Lindsay Composer (Composer) is a component-based computational framework that integrates heterogeneous computational engines and visualization techniques within a single simulation context. The Composer allows one to build computational models in a threedimensional virtual space creating complex scenes for demonstration, exploration, and investigation of physical scenarios. Composer presents simulations as a hierarchy of components. A component (in context of the Composer) can be defined as a unit of independent deployment [61].

Figure A.1A shows a component hierarchy for a cube in a simulation. The camera is a view point into a scene that can be controlled by a user through its user interaction component (i.e., the user moving the camera in the simulation). The transform component defines origin, scale, and orientation of the camera. The graphics component gives a camera a mesh, a three-dimensional shape. Given that a simulation can have multiple cameras, a given camera can be used to see other cameras in the scene which are visible by their graphics 
(mesh) representation. Figure A.1B presents the visual representation of the cube which is a direct result of a cube's component hierarchy with the graphics component responsible for visualizing the cube on the screen.
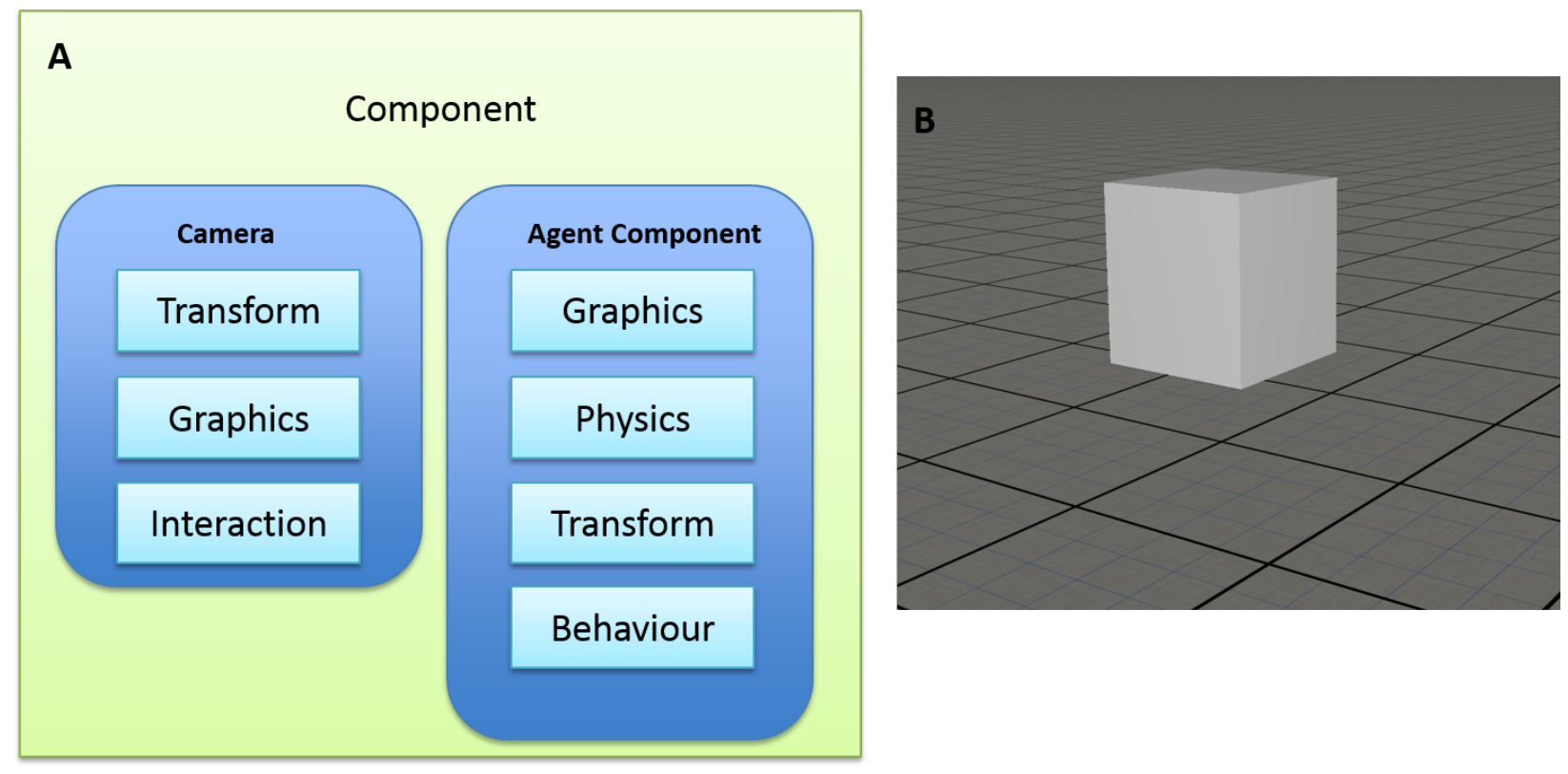

Figure A.1: Component hierarchy and structure of a cube (A) and the cube's visual appearance in a scene $(B)$ viewed through a camera. The scene includes graphics, physics and transform information of a cube in simulation space.

Components in a simulation are discrete units that can communicate directly or indirectly with other components. Let's take our cube example, in which the transform component has the location of the box in $\mathrm{x}, \mathrm{y}$, and $\mathrm{z}$ coordinates. This location can be modified by the physics component through computation of physical interactions. As the physics component updates the $\mathrm{x}, \mathrm{y}$ and $\mathrm{z}$ coordinates of the cube, the graphics component constantly updates the cube's visual representation on the screen based coordinates from the transform component. In this scenario, the physics component indirectly is updating the visual aspect of the cube via the transform component (Figure A.2. 


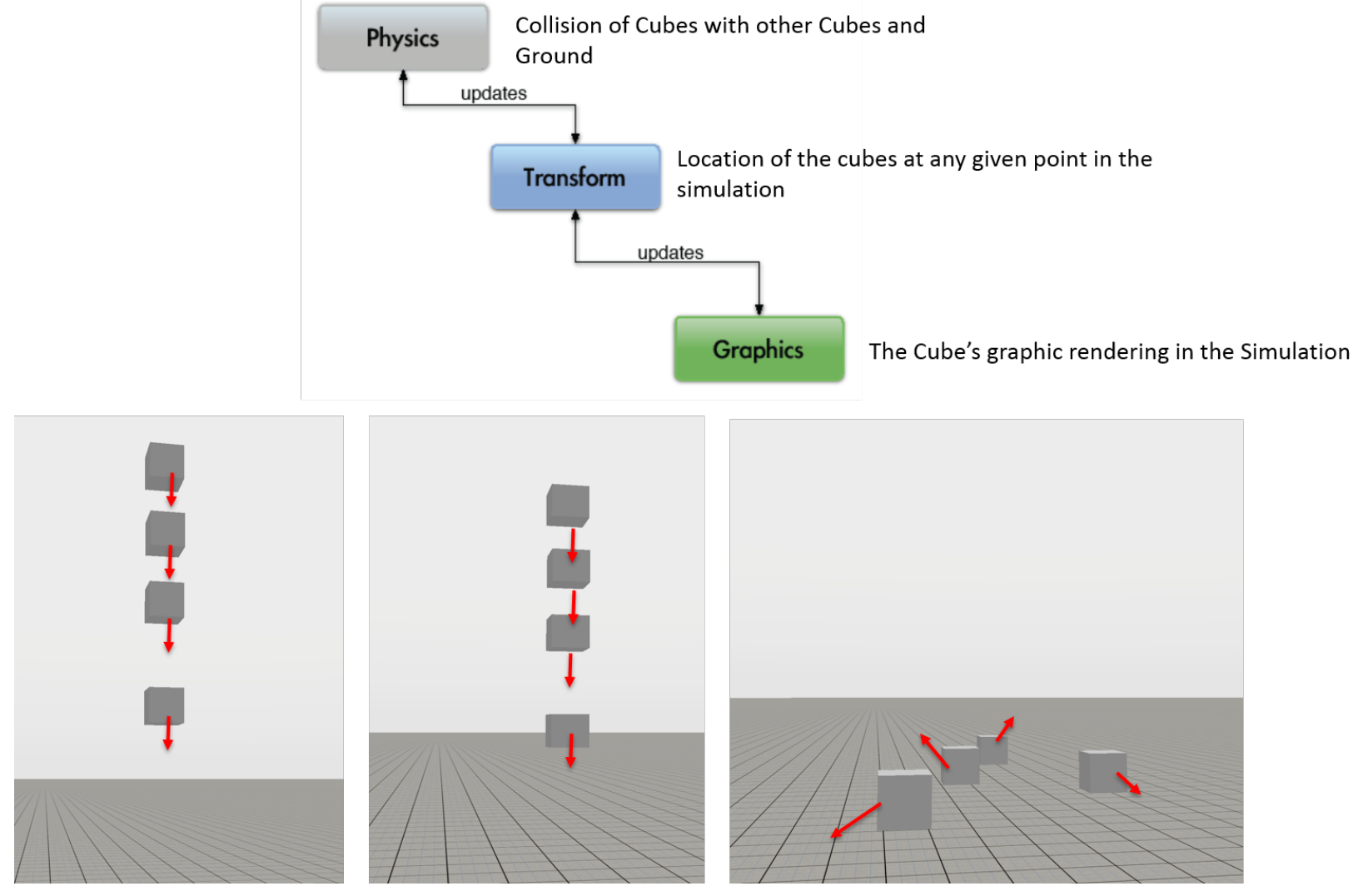

Figure A.2: Components can communicate with each other. For example, physics component can update the graphics indirectly through a transform component. The update is bidirectional and the graphics component could update the transform which in turn will result in the physics to be updated. The Scene in the Figure illustrates the Physics, Transform, and Graphics interaction while the simulation is running. 


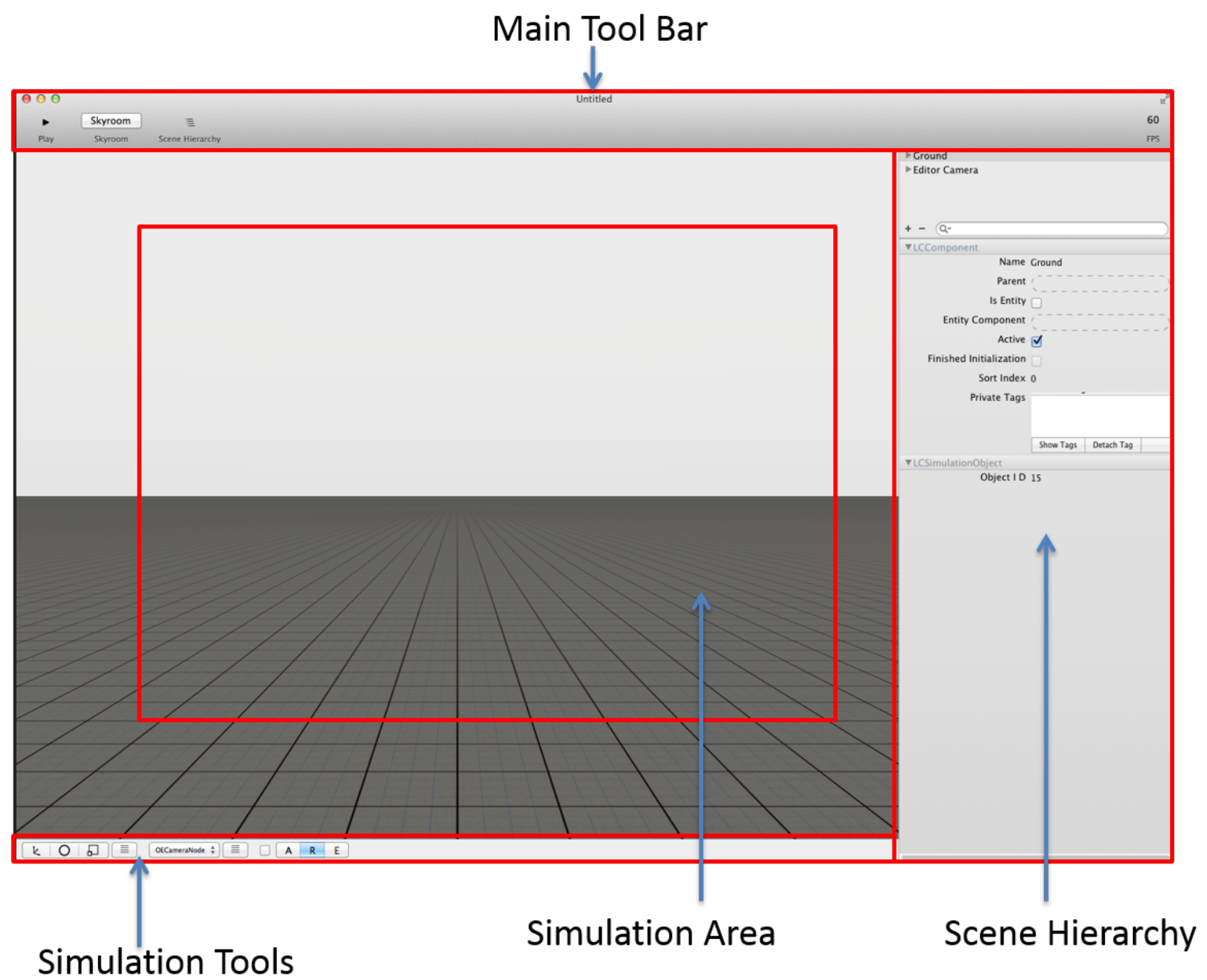

Figure A.3: Screenshot of Lindsay Composer's simulation environment. The interface is composed of a toolbar, scene and simulation area. 


\section{Appendix B}

\section{Rendering \& Visualization}

Lindsay Composer's graphics engine is responsible for high-end visualization within the simulation space. Composer uses OGRE (Object-oriented Graphics Rendering Engine) 3D [59], an open source scene-oriented, flexible 3D engine written in $\mathrm{C}++$. OGRE is designed for 3D visualization and utilizes 3D hardware available on todays graphic cards. Composer provides facilities (wrappers) to interface with OGRE libraries and utilizes OGRE's features that use underlying system libraries such as OpenGL. Composer's graphics component communicate directly with OGRE to draw three-dimensional models on the screen.

The step in simulating a bacterium is to visualize its cellular structure. This is achieved through Composer's "Mesh" component (with class name OEMeshNode) that takes in a mesh data as a resource and renders the model in three-dimensional simulation space. Mesh component has a resource parameter that points to a 3D mesh file to be loaded on screen. OGRE uses '.mesh' file format for importing 3D models into a scene.

\section{B.1 Protein Visualization}

Proteins are building blocks of cells and participate in critical cell functions. Chemotaxis is operated and controlled by several key proteins, in our model we cover the core proteins involved. The list of these proteins is as follows:

- Chemotaxis Protein A and W complex (Figure B.1A)

- Chemotaxis Protein B (Figure B.1B and C)

- Chemotaxis Protein R (Figure B.1D)

- Chemotaxis Protein Y (Figure B.1E and F) 
- Chemotaxis Protein Z (Figure B.1H and I)

- Chemotaxis Receptor Complex (Figure B.1G)

The role of each protein was reviewed in Chapter 2.1. These proteins are key agents that control and operate the bacterium's chemotactic behaviour. CheB, CheY, and CheZ come in forms of phosphorylated and non-phosphorylated states as seen in Figure B.1. In this work, an effort was made to visualize these proteins based on scientific data available on their structural shapes. The structure of these proteins (Figure B.1) were imported from the RCSB Protein Data Bank (PDB http://www.rcsb.org/), a worldwide archive of structural data of proteins.
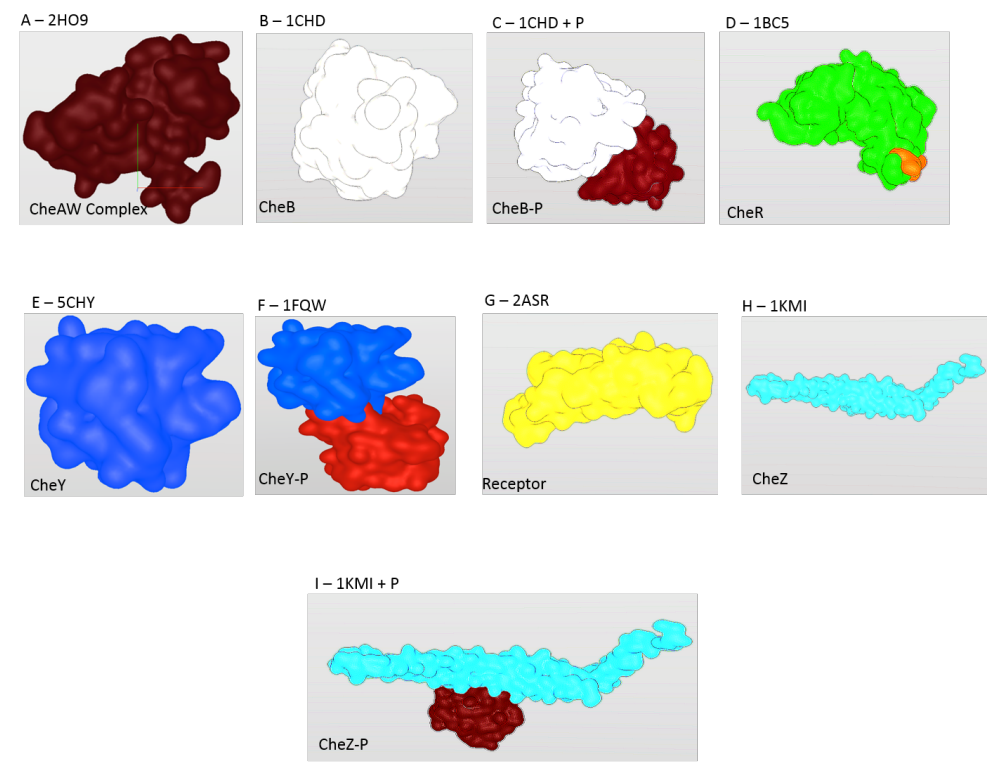

Figure B.1: Three-Dimensional structure of Chemotaxis proteins and their corresponding PDB ID number.

\section{B.1.1 Importing PDB Files}

The pipeline of importing a three-dimensional structure of a protein into the simulation environment is as follows (Figure B.2): 
1. Obtain the appropriate PDB ID for the protein by navigating to the RCSB PDB website (http://www.rcsb.org) and looking up the ID by searching the database. For example, CheY protein - 5CHY.

2. Use the obtained PDB ID to import the file to Autodesk Maya using mMaya toolkit [48]. Figure B.3A shows mMaya's import tab where one can either select a currently downloaded PDB file from a local library or download the PDB by inputting the protein's PDB file and clicking on download.

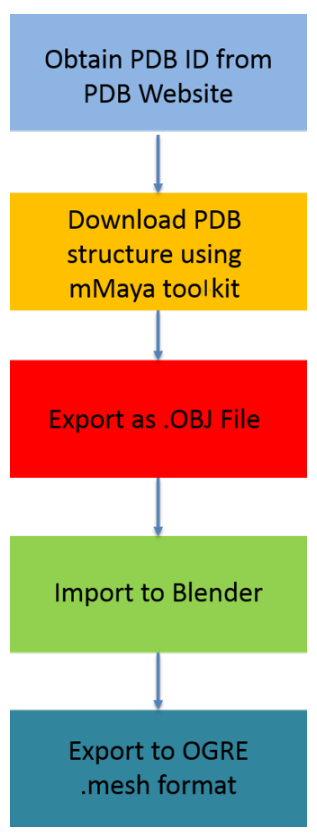

Figure B.2: Steps required to convert a PDB file to OGRE mesh.

3. Select the proper visualization (mesh vs atoms). Figure B.3B shows mMaya's atom and mesh options. In this figure, mesh visualization is selected. Figure B.4 shows a mesh visualization for a protein after importing the PDB to Maya using mMaya toolkit.

4. Export the protein mesh as an .OBJ file

5. Import the protein .OBJ file into Blender [16] 
6. Export the .OBJ file to OGRE (refer to section C.1 for details on exporting a three-dimensional object from Maya to OGRE).

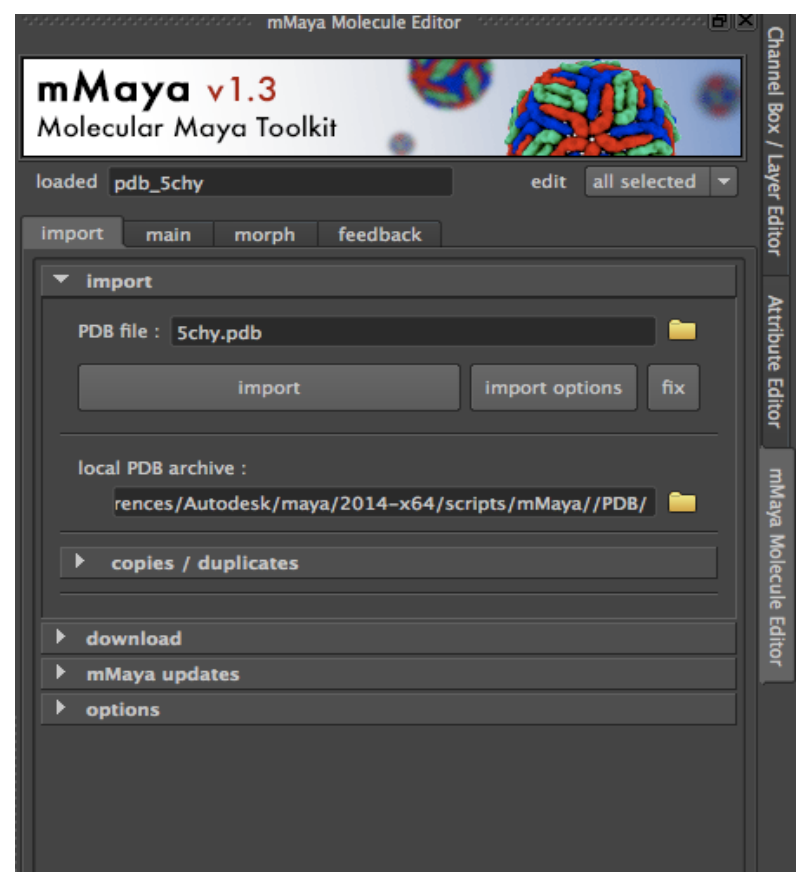

A

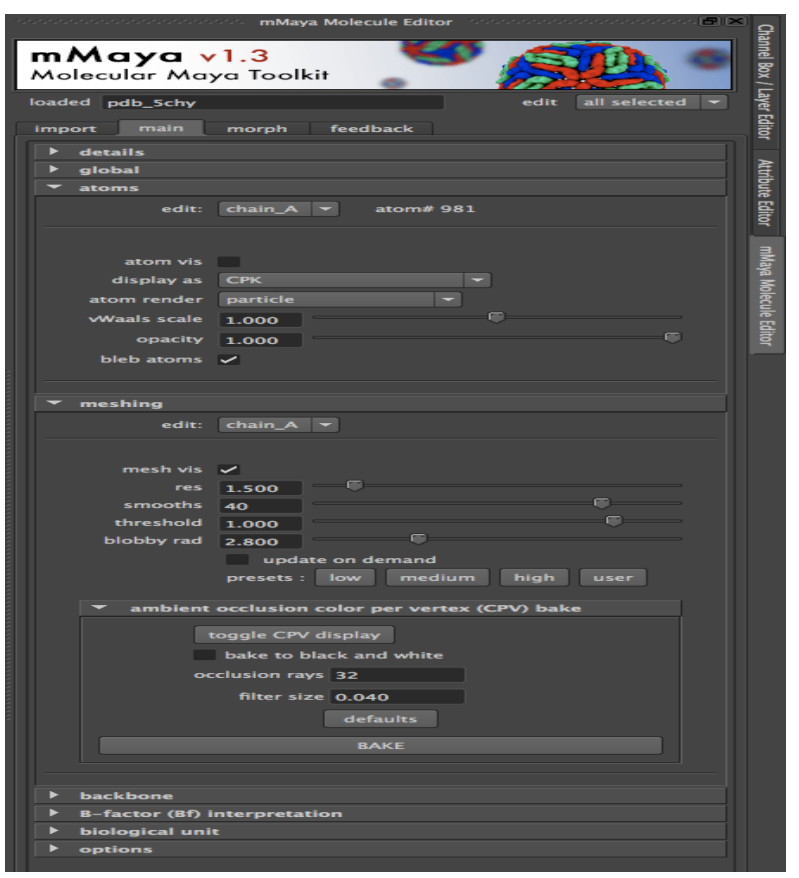

B

Figure B.3: Screenshot of mMaya import tab toolkit (A). mMaya's protein tab (B) allows for different visualization (mesh vs atoms) options to render a protein structure on the screen. 


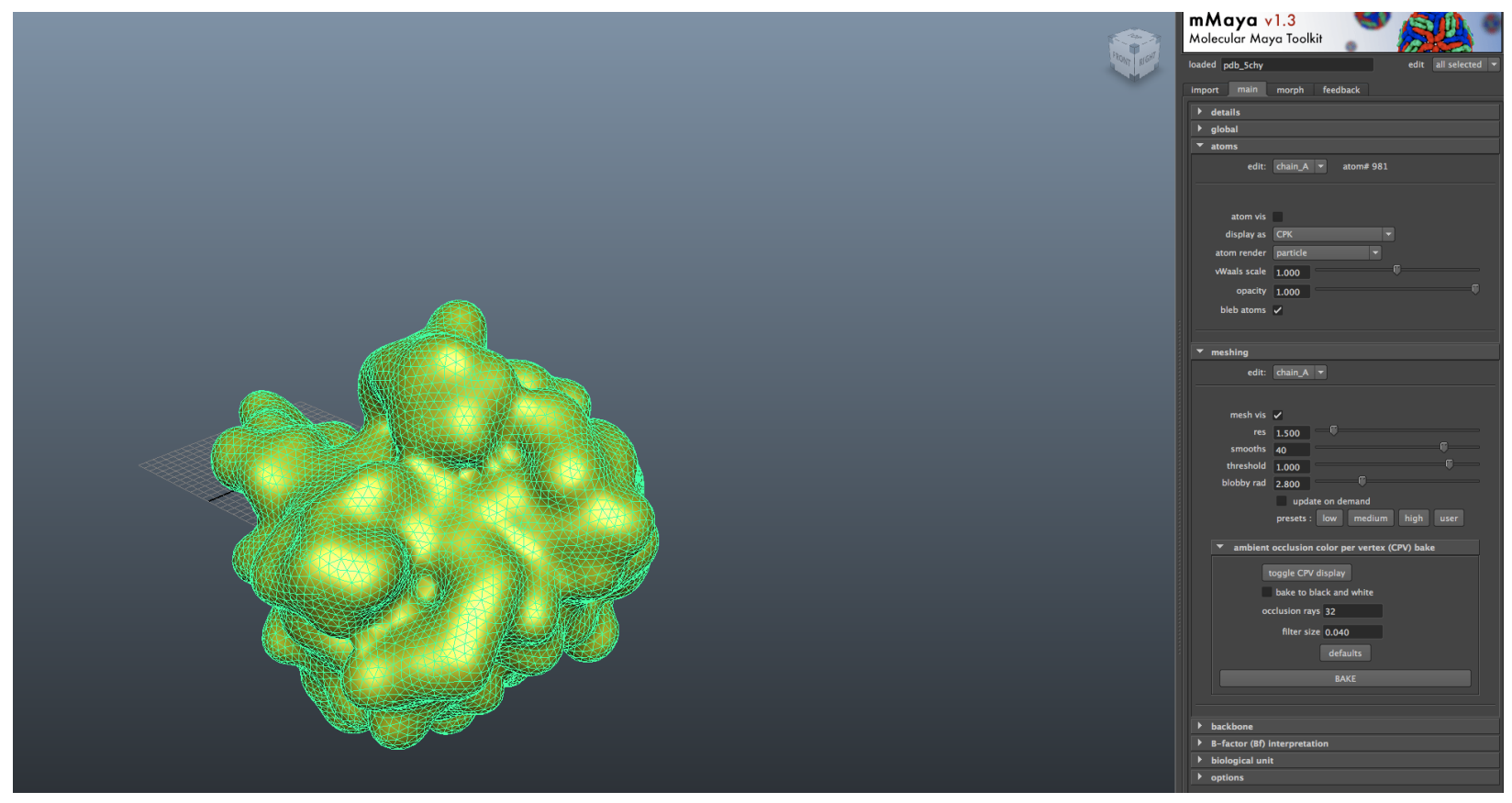

Figure B.4: Screenshot of a CheY Protein rendered on screen in Autodesk Maya after importing a PDB file through mMaya toolkit. 


\section{Appendix C}

\section{Importing 3D Models}

\section{C.1 Importing PDB Structures Into Lindsay Composer}

A three-dimensional model is constructed in a 3D modelling tool by an artist. The appearance of the model then is assigned through a set of materials (colour, specular, normals, ambient, UV maps and etc).

The three-dimensional models used in the simulation presented in this thesis, were imported using two major 3D modelling tools: Autodesk Maya [11] and Blender [16]. Autodesk Maya is a commercial product and is not available freely (with an exception of student version [10]), and Blender is an open-source project and is licensed under GNU General Public License.

Both Autodesk Maya and Blender do not natively support exporting 3D models to OGRE's Mesh format. They require a plugin for this to work. In order to properly export the 3D models to OGRE's Mesh format to be used in the Lindsay environment, Maya and Blender were used sequentially to generate the .mesh file of the 3D protein structure.

The OGRE community has developed its own Blender exporter in Python script [46]. The script works both for Blender on Mac and Windows machines however does not come with a simple installer and both versions require the user to place the script in a proper directory manually.

The Composer simulation environment has a list of OGRE model directories that it scans upon start for loading resources which become available after application has finished launching. The newly created mesh has to be located inside a folder that is already been added as a resource to the simulation environment, or it can be added as a new directory manually. Figure C.1 shows the Composer's resource manager (the plugin is called Omija) 
listing all the directories in the system which is indexed for resources. One could add a folder manually by clicking on the plus $(+)$ button at the lower left of the windows. Unwanted resources can also be removed by the user through the minus (-) button 1 .

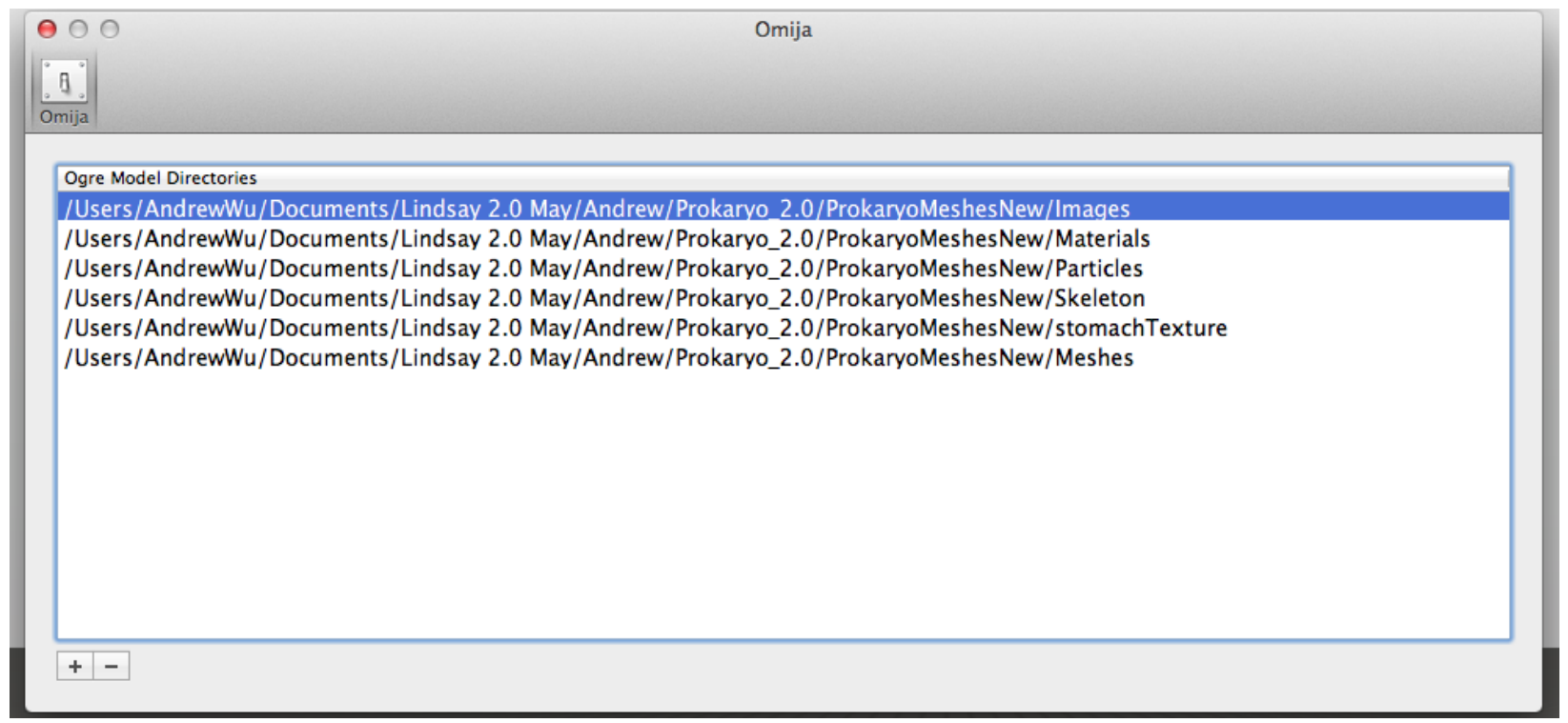

Figure C.1: A screenshot of OGRE media directories loaded by the Composer's resource manager.

\section{C.2 Model Appearance}

The appearance of a three-dimensional model in the simulation is specified by material scripts. A material script defines rendering options for a 3D mesh by providing information about what textures, patterns, colours, and so forth should be applied to a surface. Colours or patterns applied to a mesh is not sufficient in producing a proper visualization. The lighting of the model is also crucial in producing a visually appealing model. As an example, real world objects reflect light depending on their material (quality of surface). The material script provides all the information necessary for the graphics engine to render the object on the screen.

\footnotetext{
${ }^{1}$ The resource manager window can be accessed through the Composer's main menu: LindsayComposer $\rightarrow$ Preferences.
} 
The OGRE graphics engine scans all resource locations and parses all files with '.material' extension. A single material script could contain multiple materials for multiple 3D models. The name of the material file is not as important since the graphics engine looks for a material extension. Every material within the material file has a name. This is the name the graphics engine uses to match a given material to its appropriate model. For our example in Figure C.2, the protein CheY (PDB - 5CHY) has a mesh file and a material file, the mesh file points to the material file, and in the material file it defines the rendering options for the CheY protein. Figure C.2A is the result of what we see in our simulation environment. 


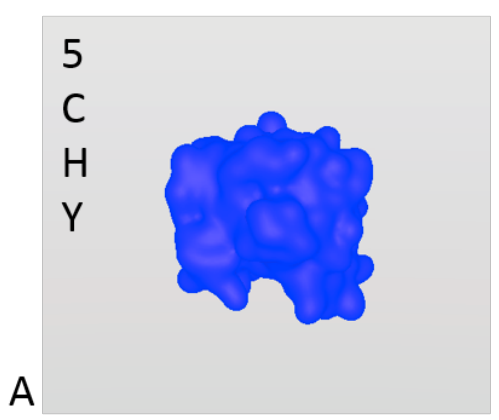

\section{B}

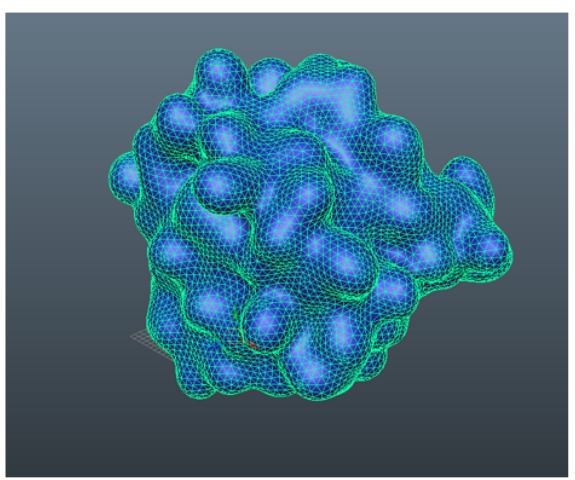

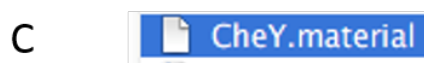

material pdb_1faw_chain_A_surfshaderSG.001 receive_shadows on technique

pass pdb_1fqw_chain_A_surfshaderSG.001

ambient $0.18764051795005798 \quad 0.218737840652465820 .89999997615814210 .0$ diffuse $0.150112416596895670 .174990275129530910 .7199999916553494 \quad 0.0$ specular $0.250 .25 \quad 0.25 \quad 0.012 .5$

alpha_to_coverage off colour_write on

cult hardware clockwise

depth_check on

depth_func less_equal

illunination_stage

light_clip_planes off

light_scissor off

lighting on

normalise_normals of

scene blond solid

scene_blend_op add

shading gouraud

transparent_sorting on

Figure C.2: A example of the mesh and material files associated and required to render a Protein (CheY) in our LINDSAY Composer environment. A) The rendered result of the CheY protein in the simulation. B) The mesh file that defines the mesh data and points to the material file that defines rendering options. C) A snippet of the material script for the CheY protein. 